University of Tennessee Health Science Center

UTHSC Digital Commons

\title{
CD151 Reinforces Vascular Stability by Balancing Endothelial Cell Adhesion and Cytoskeletal Tension
}

\author{
Feng Zhang \\ University of Tennessee Health Science Center
}

Follow this and additional works at: https://dc.uthsc.edu/dissertations

Part of the Medical Cell Biology Commons, Medical Microbiology Commons, and the Neoplasms Commons

\section{Recommended Citation}

Zhang, Feng, "CD151 Reinforces Vascular Stability by Balancing Endothelial Cell Adhesion and Cytoskeletal Tension" (2010). Theses and Dissertations (ETD). Paper 322. http://dx.doi.org/10.21007/ etd.cghs.2010.0374.

This Dissertation is brought to you for free and open access by the College of Graduate Health Sciences at UTHSC Digital Commons. It has been accepted for inclusion in Theses and Dissertations (ETD) by an authorized administrator of UTHSC Digital Commons. For more information, please contact jwelch30@uthsc.edu. 


\title{
CD151 Reinforces Vascular Stability by Balancing Endothelial Cell Adhesion and Cytoskeletal Tension
}

\author{
Abstract \\ Tetraspanin CD151 is highly expressed in endothelial cells and regulates pathological angiogenesis. \\ However, the mechanism by which CD151 promotes vascular morphogenesis and whether CD151 \\ engages other vascular functions are unclear. We observed that CD151 is required for the maintenance of \\ endothelial capillary-like structures formed in vitro and the integrity of lung endothelial cell-cell contacts in \\ vivo. As a master regulator of endothelial cell-matrix and cell-cell adhesions, CD151 is needed for the \\ optimal functions of various cell adhesion proteins such as integrin, cadherin, and CD44. The loss of \\ CD151 elevates the cellular intrinsic contraction by upregulating RhoA signaling and downregulating of \\ Rac1 activity. CD151 balances RhoA and Rac1 activities and stabilizes the membrane-cytoskeleton \\ connection. In the absence of CD151 expression, the inhibition of RhoA signaling axis enhances the \\ diminished cell-cell adhesiveness and stabilizes vascular structures. In addition, CD151-dependent \\ vascular stabilization involves cAMP signaling and might thus regulate RhoA and Rac1. Together, CD151 \\ reinforces vascular stability by promoting endothelial cell-matrix and cell-cell adhesions to \\ counterbalance the destabilization derived from RhoAsignaling-mediated cytoskeletal tension.

\section{Document Type} \\ Dissertation \\ Degree Name \\ Doctor of Philosophy (PhD) \\ Program \\ Biomedical Sciences \\ Research Advisor \\ Xin A. Zhang, M.D., Ph.D. \\ Keywords \\ CD151, cell adhesion, cytoskeletal tension, Rho GTPases, tetraspanin, vascular stability

\section{Subject Categories} \\ Diseases | Medical Cell Biology | Medical Microbiology | Medical Sciences | Medicine and Health Sciences \\ | Neoplasms

\section{Comments} \\ Three year embargo expired December 2013
}




\title{
CD151 REINFORCES VASCULAR STABILITY BY BALANCING ENDOTHELIAL CELL ADHESION AND CYTOSKELETAL TENSION
}

\author{
A Dissertation \\ Presented for \\ The Graduate Studies Council \\ The University of Tennessee \\ Health Science Center \\ In Partial Fulfillment \\ Of the Requirements for the Degree \\ Doctor of Philosophy \\ From The University of Tennessee
}

By

Feng Zhang

December 2010 
Copyright (C) 2010 by Feng Zhang All rights reserved 


\section{DEDICATION}

This dissertation is dedicated to my parents,

Xingshun Zhang and Shaoxiang Chen, and my brother Ning Zhang, for their love and support. 


\section{ACKNOWLEDGEMENTS}

I would like to express my most sincere thanks to my advisor, Dr. Xin A. Zhang for his guidance and full support throughout my Ph.D. studies. Dr. Zhang has been teaching me to develop the ideas and experimental designs for scientific research and complete the challenging thoughts that lie behind it. I also thank Dr. Zhang for all his guidance in resolving problems in the project and his patient training in scientific writing. Dr. Zhang was always there to listen and give me advice and encouragement not only in the research work, but also in things aside from science.

I would also like to express my deep gratitude to the rest of my Ph.D. committee, Dr. Suzanne J. Baker, Dr. Jill M. Lahti, Dr. Anjaparavanda P. Naren and Dr. Lawrence M. Pfeffer for their guidance, encouragement and valuable suggestions. I especially thank Dr. Jill Lathi for her generous help with the time lapse video microscopy, TIRF microscopy and electron microscopy studies.

I gratefully acknowledge Dr. Hayden Huang and his student Jarett Michaelson from Columbia University for collaborating with us on the traction force microscopy studies. Thanks also to Simon Moshiach from St. Jude Children's Research Hospital for collaborating with us on the time lapse video microscopy, Dr. Yi Zheng from Cincinnati Children's Hospital Medical Center for providing the Rac1 F28L retrovirus and Dr. Wenyuan Zhao, Dr. Yuanjian Chen and Dr. Yao Sun from Dept. of Medicine, UTHSC for collaborating with animal studies. I appreciate Dr. Arnoud Sonnenberg from the Netherlands Cancer Institute for providing CD151+/- mice and Dr. Christopher Stipp from University of Iowa for providing CD151 knockdown constructs.

I am grateful to my dear labmates Mekel Richardson and Yanhui Zhang for their valuable collaborations, inspiring discussion and friendship.

I thank my parents: my father Xingshun Zhang and my mother Shaoxiang Chen, for giving me life in the first place and for their everlasting love and unconditional support, and my brother Ning Zhang for his support and encouragement.

During the course of my Ph.D. studies, I was supported in part by the predoctoral fellowship from American Heart Association 09PRE2260283. I appreciate the financial support from American Heart Association. 


\begin{abstract}
Tetraspanin CD151 is highly expressed in endothelial cells and regulates pathological angiogenesis. However, the mechanism by which CD151 promotes vascular morphogenesis and whether CD151 engages other vascular functions are unclear. We observed that CD151 is required for the maintenance of endothelial capillary-like structures formed in vitro and the integrity of lung endothelial cell-cell contacts in vivo. As a master regulator of endothelial cell-matrix and cell-cell adhesions, CD151 is needed for the optimal functions of various cell adhesion proteins such as integrin, cadherin, and CD44. The loss of CD151 elevates the cellular intrinsic contraction by upregulating RhoA signaling and downregulating of Rac1 activity. CD151 balances RhoA and Rac1 activities and stabilizes the membrane-cytoskeleton connection. In the absence of CD151 expression, the inhibition of RhoA signaling axis enhances the diminished cell-cell adhesiveness and stabilizes vascular structures. In addition, CD151-dependent vascular stabilization involves cAMP signaling and might thus regulate RhoA and Rac1. Together, CD151 reinforces vascular stability by promoting endothelial cell-matrix and cell-cell adhesions to counterbalance the destabilization derived from RhoA signaling-mediated cytoskeletal tension.
\end{abstract}




\section{TABLE OF CONTENTS}

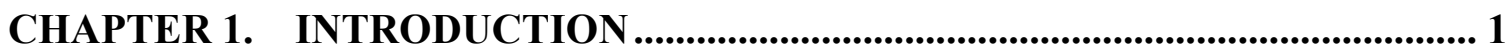

1.1 Vascular Morphogenesis and Vascular Stability ..................................................... 1

1.1.1 An Introduction to Vascular Morphogenesis ............................................... 1

1.1.2 Cell-matrix Adhesion, Cell-cell Adhesion, and Cytoskeletal Organization

Are Important for Vascular Morphogenesis and Vascular Stability............... 1

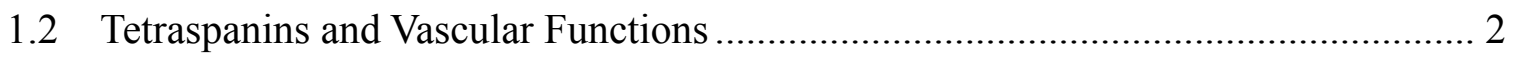

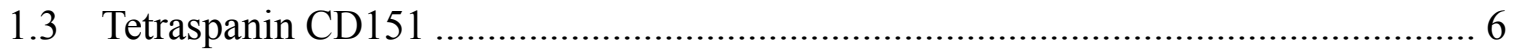

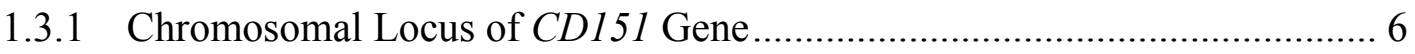

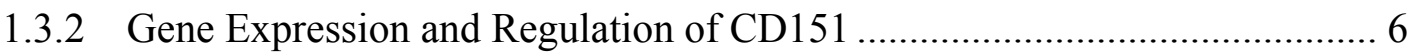

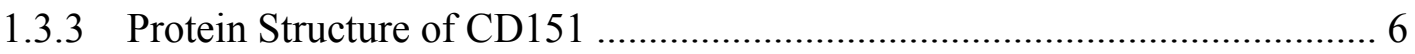

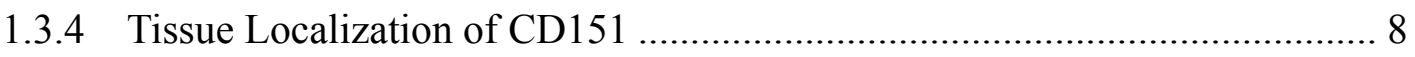

1.3.5 Association of CD151 with Integrins and Other Tetraspanins ...................... 8

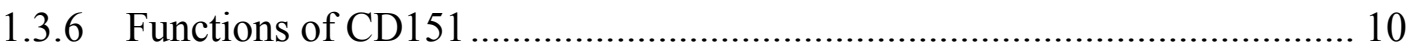

1.3.6.1 The Roles of CD151 in Integrin-dependent Functions ................. 10

1.3.6.2 CD151 Is a Tumor Metastasis Promoter ........................................11

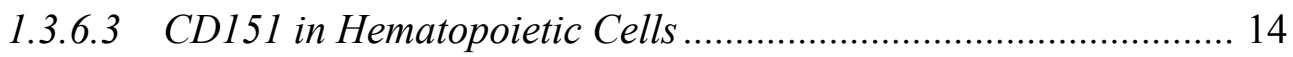

1.3.6.4 CD151 in Vascular Endothelium and Its Role in Angiogenesis.... 15

1.3.7 CD151 Deficiency in Human and Mouse ..................................................... 16

1.3.7.1 CD151 Deficiency-related Disease in Human............................. 16

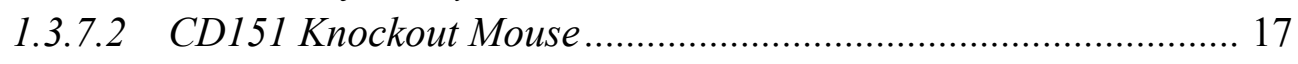

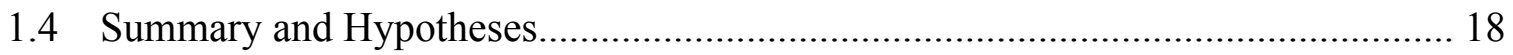

\section{CHAPTER 2. CD151 STABILIZES VASCULAR NETWORK} STRUCTURES AND CONFINES VASCULAR PERMEABILITY............................. 20

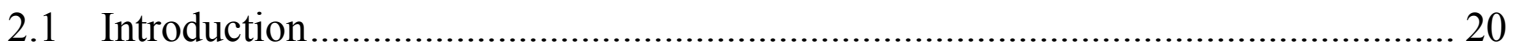

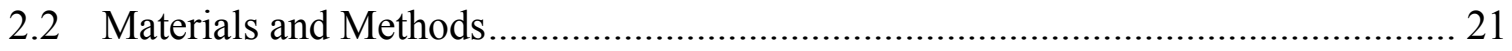

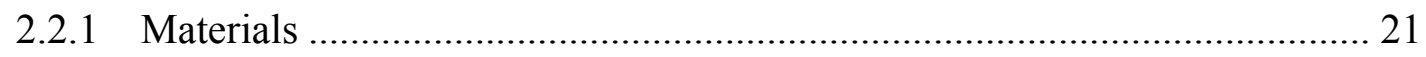

2.2.2 Cell Culture and CD151-null Mice..................................................... 21

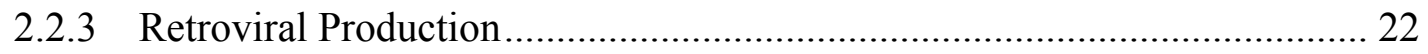

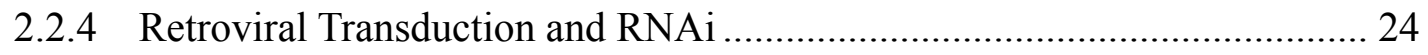

2.2.5 Flow Cytometry and Immunofluorescence................................................. 24

2.2.6 3D Capillary Formation Assay............................................................ 24

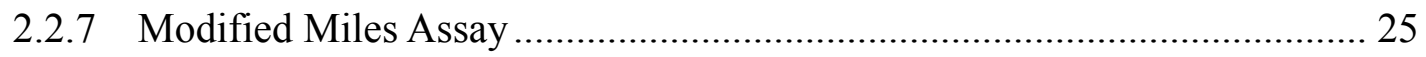

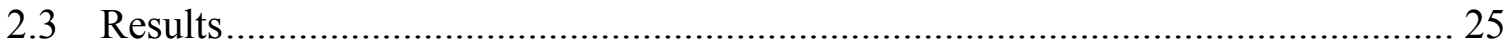

2.3.1 Silencing CD151 Expression in Endothelial Cells ..................................... 25 
2.3.2 CD151 Silencing Does Not Alter Cell Surface Expression of CD151 -related Integrins and Tetraspanins ........................................................... 27

2.3.3 Identification of Isolated MLECs .............................................................. 27

2.3.4 CD151 Stabilizes Vascular Network Structures Formed by Endothelial

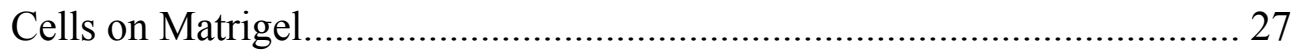

2.3.5 CD151 Regulates Vascular Permeability ................................................... 31

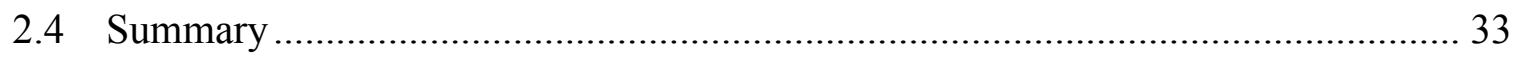

\section{CHAPTER 3. CD151 STRENGTHENS ENDOTHELIAL CELL-MATRIX ADHESION WITHOUT AFFECTING CELL DEATH AND MOTILITY .............. 34}

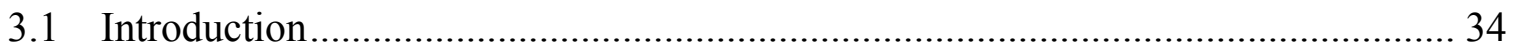

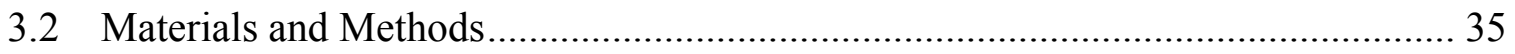

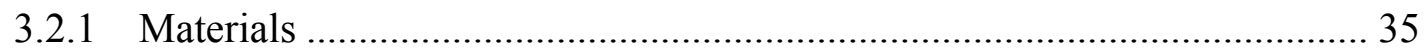

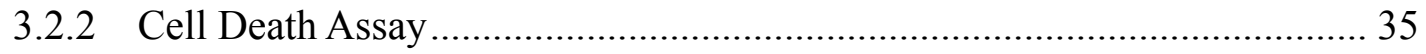

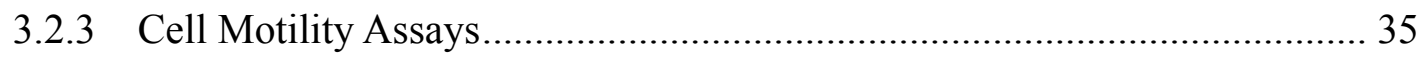

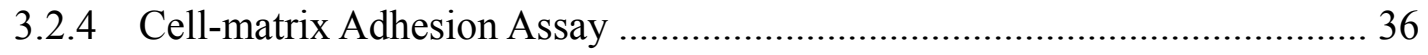

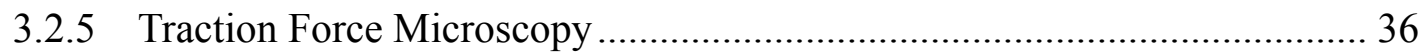

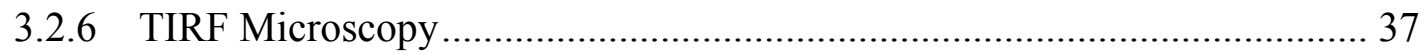

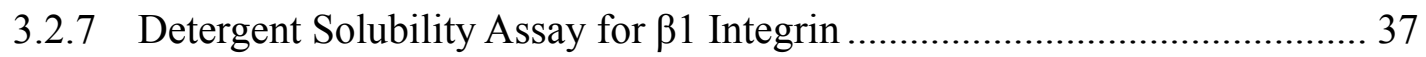

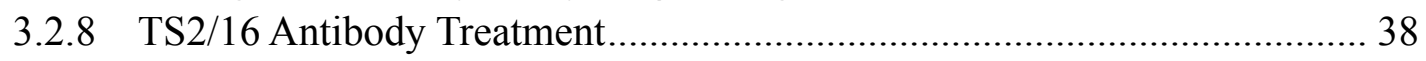

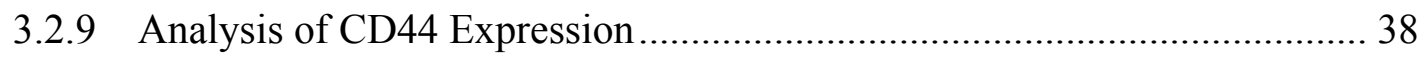

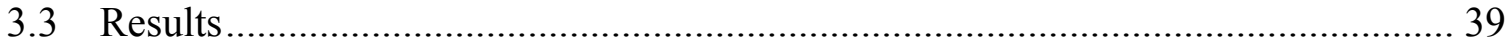

3.3.1 CD151 Does Not Affect Cell Death........................................................... 39

3.3.2 Endothelial Cell Migration Remains Unchanged in the Absence of

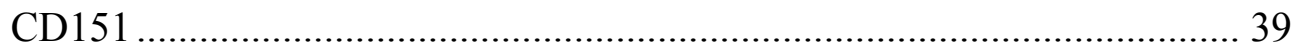

3.3.3 CD151 Is Needed for Optimal Endothelial Cell-matrix Adhesiveness ....... 39

3.3.4 CD151 Does Not Determine Physical Properties of $\alpha 3$ Integrin and CD9 in Endothelial Cell-matrix Interface.................................................... 43

3.3.5 CD151 Regulates Glycosylation of $\alpha 3$ Integrin............................................ 43

3.3.6 CD151 Strengthens the Connection of $\beta 1$ Integrin to Actin Cytoskeleton.. 46

3.3.7 Reinforcement of Cell-matrix Adhesion Partially Rescues the Defective Vascular Network Structures Formed by CD151-silenced Endothelial

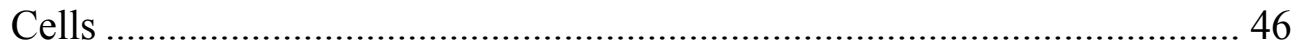

3.3.8 CD151 Regulates CD44 Expression in Endothelial Cells ……………….... 49

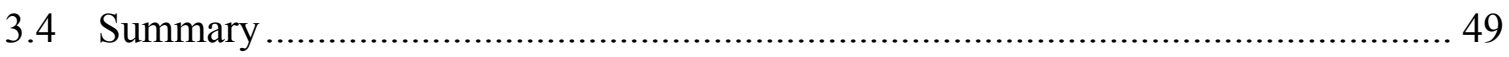

\section{CHAPTER 4. CD151 REINFORCES ENDOTHELIAL CELL-CELL} ADHESION .

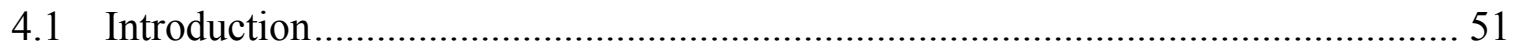




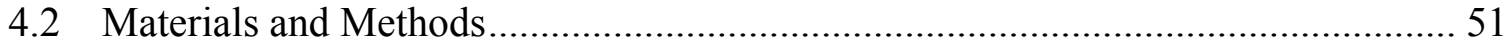

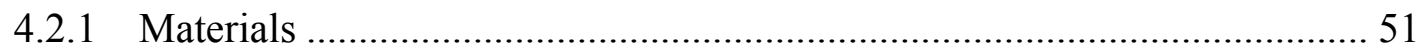

4.2.2 Cell Aggregation Assay ……………………….................................... 52

4.2.3 Analysis of Cellular Distribution Adherens Junctional Complex................. 52

4.2.4 Transmission Electron Microscopy (TEM) ……………………..............52

4.2.5 Analysis of Protein Association of Adherens Junctional Complex ............. 52

4.2.6 Detergent Solubility Assay for VE-cadherin .............................................. 53

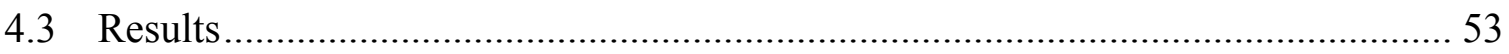

4.3.1 CD151 Strengthens Calcium-dependent Cell-cell Adhesion in

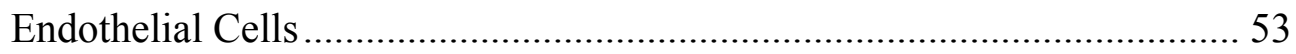

4.3.2 CD151 Stabilizes VE-cadherin-mediated Adherens Junctions ..................... 55

4.3.3 CD151 Is Not Required for the Assembly of Adherens Junctional Complexes in Endothelial Cells................................................................ 55

4.3.4 CD151 Reinforces the Cytoskeletal Connection of VE-cadherin................. 57

4.3.5 CD151 Regulates VE-cadherin-independent Cell-cell Adhesiveness in

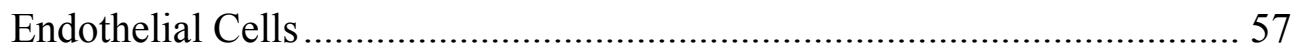

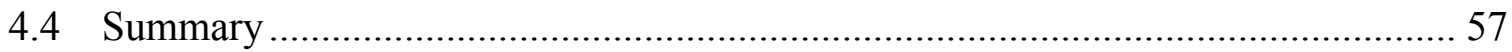

\section{CHAPTER 5. CD151 MODULATES SMALL GTPASES RAC1 AND RHOA SIGNALINGS AND RESTRAINS ENDOTHELIAL CYTOSKELETAL TENSION...}

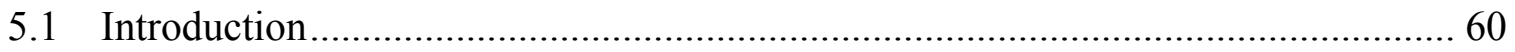

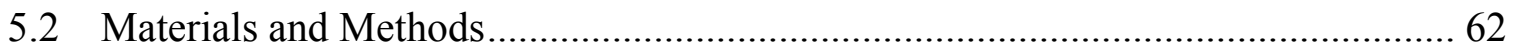

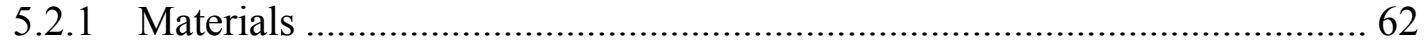

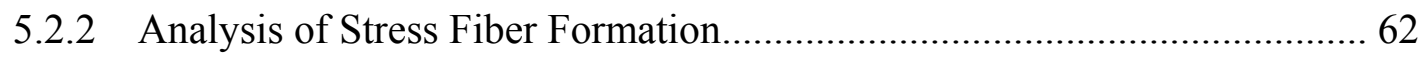

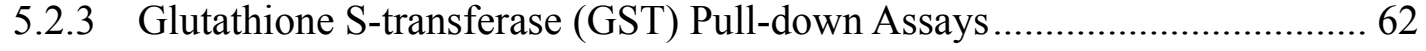

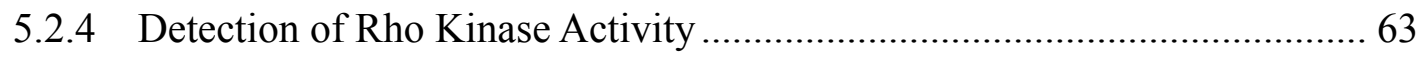

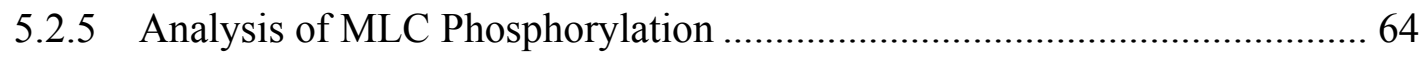

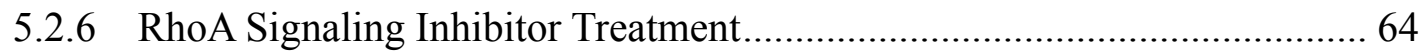

5.2.7 Analysis of Upstream Signaling of RhoA and Rac1 ….................................. 64

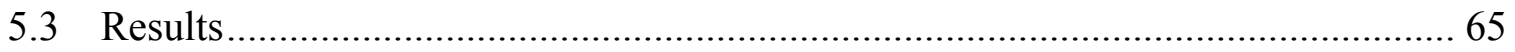

5.3.1 CD151 Restrains Stress Fiber Formation in Endothelial Cells.................... 65

5.3.2 CD151 Balances the Activations of Rac1 and RhoA Signalings ................. 65

5.3.3 RhoA Signaling Inhibitors Restore the Unstabilization of Vascular Network Structures Caused by Loss of CD151 ........................................... 68

5.3.4 Elevation in Intracellular cAMP but Not Active Rac1 Can Rescue Unstabilized Vascular Network Structures Formed in CD151 KD Cells .... 68

5.3.5 CD151-mediated Restraint on RhoA Is Not Dependent on P190Rho ${ }^{\text {GAP }}$

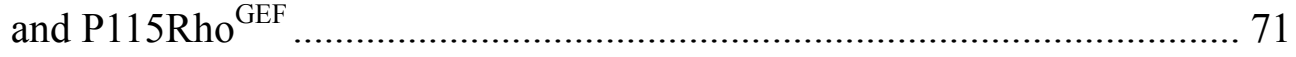

5.3.6 CD151 Downregulates Slit2 Expression ................................................. 74 


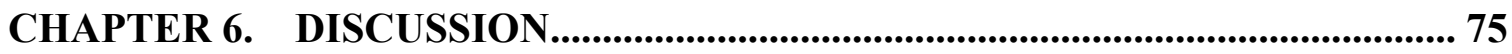

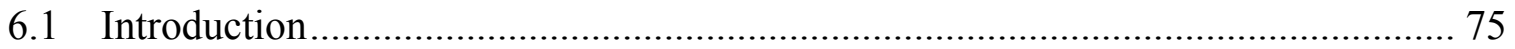

6.2 Tetraspanin CD151 Promotes Vascular Stability ………………………….......... 75

6.3 CD151 Regulates Multiple Endothelial Cell Events .............................................. 77

6.3.1 CD151 Does Not Affect Endothelial Cell Death and Cell Motility............. 77

6.3.2 CD151 Optimizes Endothelial Cell-matrix Adhesion..................................... 78

6.3.3 CD151 Reinforces Endothelial Cell-cell Adhesion ...................................... 79

6.3.4 CD151 Modulates RhoA Signaling-mediated Endothelial Cytoskeletal

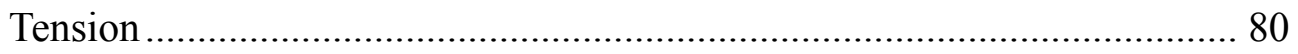

6.3.5 CD151 Might Be an Important Regulator of Cortical Cytoskeletal

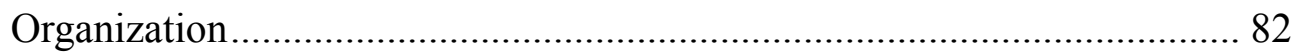

6.4 CD151 Coordinates Cell Adhesions and Actin Cytoskeletal Tension ..................... 84

6.4.1 CD151 Strengthens Cell-cell and Cell-matrix Adhesions and Regulates

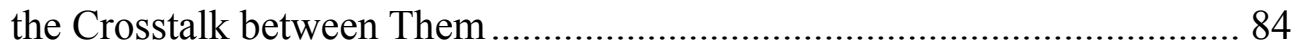

6.4.2 CD151 Regulates Cell-cell Adhesion and Cytoskeletal Tension .................. 85

6.4.3 CD151 Balances RhoA and Rac1 Signalings ……...................................... 85

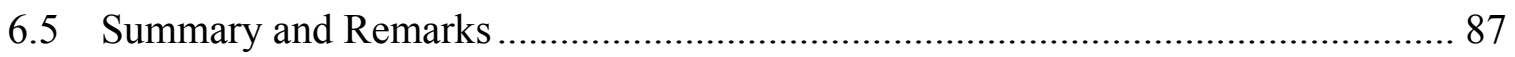

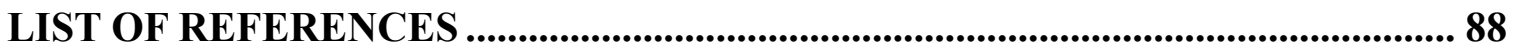

VITA 


\section{LIST OF TABLES}

Table 2-1. The surface expression of integrins and tetraspanins on HMEC-parental,

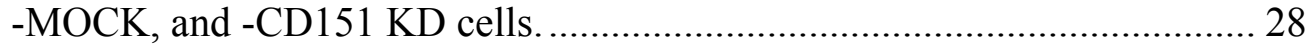

Table 3-1. Quantitative results of Matrigel cable formation assay with TS2/16

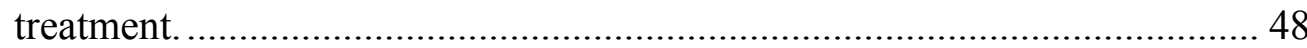




\section{LIST OF FIGURES}

Figure 1-1. Schematic representation of the structure of tetraspanins........................... 3

Figure 1-2. Schematic drawing showing cellular functions of tetraspanins .................... 5

Figure 1-3. Schematic drawing of genomic organization and protein domain structure of human CD151 ................................................................ 7

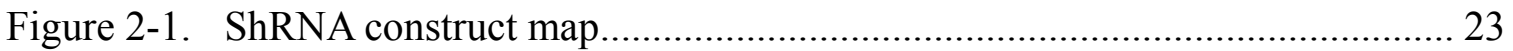

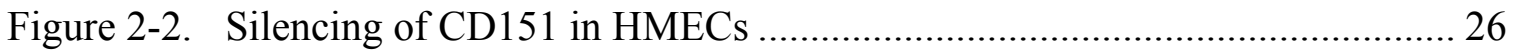

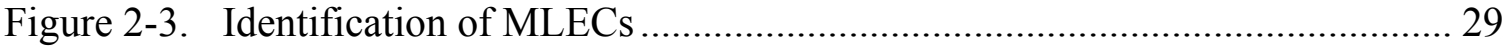

Figure 2-4. Loss of CD151 impairs vascular stability ......................................... 30

Figure 2-5. Quantification of the cable network in the time-lapse movies ................... 32

Figure 2-6. Loss of CD151 increases vascular permeability in mice ......................... 32

Figure 3-1. Loss of CD151 does not affect endothelial cell viability .......................... 40

Figure 3-2. Loss of CD151 does not alter endothelial cell migration .......................... 41

Figure 3-3. Loss of CD151 diminishes endothelial cell-matrix adhesiveness............... 42

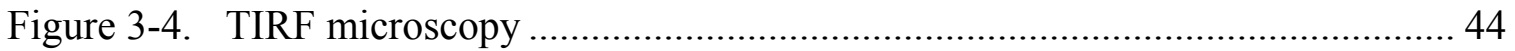

Figure 3-5. Loss of CD151 inhibits glycosylation of $\alpha 3$ integrin................................ 45

Figure 3-6. Loss of CD151 elevates detergent solubility of $\beta 1$ integrin....................... 47

Figure 3-7. TS2/16 partially rescues the defects in maintenance of capillary structures in CD151-silenced endothelial cells....................................... 48

Figure 3-8. Loss of CD151 inhibits CD44 expression .............................................. 50

Figure 4-1. Loss of CD151 impairs endothelial cell-cell adhesiveness........................ 54 
Figure 4-2. Adherens junctional complexes are mis-localized in CD151-silenced endothelial cell monolayer.......................................................................... 56

Figure 4-3. Abnormal endothelial cell-cell adhesion in lung vessels of CD151 KO mice as seen in electron micrographs 56

Figure 4-4. The protein association in adherens junctional complex is not affected

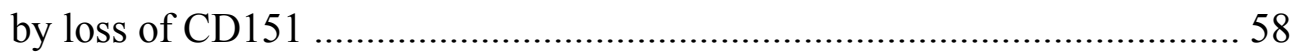

Figure 4-5. Detergent solubility assay of VE-cadherin ................................................. 59

Figure 5-1. A diagram showing RhoA and Rac1 signalings and the upstream signals .. 61

Figure 5-2. Loss of CD151 expression results in increased stress fiber formation ........ 66

Figure 5-3. Loss of CD151 expression results in upregulation of RhoA-Rho kinase-myosin signaling and downregulation of Rac1 signaling

Figure 5-4. Rho signaling inhibitors restore the defective vascular network structures caused by loss of CD151

Figure 5-5. The inhibition effect of Y27632 on Rho kinase activity 70

Figure 5-6. Elevation in intracellular cAMP but not active Rac1 rescues the defective vascular network structures caused by loss of CD151 72

Figure 5-7. Upstream signaling studies 73

Figure 6-1. A working model for CD151 in stabilizing vascular structures.................... 76

Figure 6-2. The signaling of integrin/CD151 complexes in endothelial cells 83 


\section{CHAPTER 1. INTRODUCTION}

\subsection{Vascular Morphogenesis and Vascular Stability}

\subsubsection{An Introduction to Vascular Morphogenesis}

An essential requirement for adequate organ performance is the formation of blood vessels that transport nutrients and maintain hemostasis. Vasculogenesis and angiogenesis are the two in vivo morphogenic processes of blood vessel formation. Vasculogenesis refers to the de novo development of capillaries from individual endothelial precursors, while angiogenesis refers to new blood vessel formation from preexisting vessels. ${ }^{1}$ Vascular morphogenesis is the process in which endothelial cells assemble into capillary structures and is required for both vasculogenesis and angiogenesis. ${ }^{1}$

\subsubsection{Cell-matrix Adhesion, Cell-cell Adhesion, and Cytoskeletal Organization Are Important for Vascular Morphogenesis and Vascular Stability}

During vascular morphogenesis, endothelial cells assemble into inter-connected multicellular structure by the linkage between cells through cell-cell adhesion and attachment on basement membrane through cell-matrix adhesion. ${ }^{2,3}$ The interactions between cell and matrix and among cells are critical for endothelium to resist the constant tension from the endothelial actin cytoskeleton and the shear force from blood flow. ${ }^{2,3}$

Endothelial cell-matrix adhesion is mediated by a number of specific adhesive proteins, of which integrins are of main importance. ${ }^{4}$ Integrins are a group of heterodimeric cell surface receptors composed of non-covalently associated $\alpha$ and $\beta$ subunits and bind with their extracellular matrix (ECM) ligands to mediate cell-matrix adhesion. ${ }^{5,6}$ Vessels contain various integrins such as fibronectin-binding integrin $\alpha 5 \beta 1$, and laminin-binding integrins $\alpha 3 \beta 1, \alpha 6 \beta 1$ and $\alpha 6 \beta 4 .{ }^{4}$ The integrin-matrix engagement is not only responsible for endothelial cell adhesion to matrix environment and but also involved in regulation of vascular functions. ${ }^{4}$ For example, integrin $\alpha 5 \beta 1$ is of particular importance in vascular development. ${ }^{7}$ In addition, other endothelial cell-matrix adhesion molecules such as CD44 are also important for angiogenesis., 8

Endothelial cell-cell adhesion is also crucial for blood vessel formation as the vessel wall is formed largely or partially by the endothelial cell layer in which adjacent endothelial cells attach to each other through cell-cell contacts to maintain hemostasis., ${ }^{2,3}$ 
VE-cadherin is an endothelium-specific cell-cell adhesion molecule that physically and functionally associates with $\alpha-, \beta-$, and $\gamma$-catenin, and $\mathrm{p} 120^{\mathrm{cth}}$ to form adherens junctions (AJ). ${ }^{10-12}$ VE-cadherin is essential for the morphogenesis of vessel-like structure in vitro, ${ }^{12-14}$ and the ablation of VE-cadherin or function-blocking antibodies effectively reduce neovascularization in animal. ${ }^{14,15}$ In addition to VE-cadherin, endothelial cells express other cell-cell adhesion proteins such as occludin, claudins and junctional adhesion molecule-1 (JAM-1) in tight junctions (TJ), and platelet endothelial cell adhesion molecule (PECAM), vascular cell adhesion molecule (VCAM) and intercellular adhesion molecule-1 (ICAM-1) and -2 which are not specifically confined to AJ and TJ. ${ }^{11,16}$ Among these molecules, PECAM-1 and ICAM-2 have also been implicated in the regulation of angiogenesis. ${ }^{11,16}$

Cytoskeletal organization is also important in vascular morphogenesis and vascular stability. Rho family GTPases control various cytoskeleton reorganization events such as Rac1- and Cdc42-dependent protrusions and RhoA-mediated cell contraction/retraction. ${ }^{17}$ It has been established that Rac1, Cdc42, and RhoA are important for the vessel structure formation. ${ }^{1,18-21}$ For example, Rac1 and Cdc42 activations are needed for angiogenesis, ${ }^{22,23}$ and are specifically required for the formation of capillary lumen in 3D extracellular matrices. ${ }^{1,18,24}$ In contrast, the activation of RhoA destabilizes angiogenic vessels, and is required for vessel regression, whereas either the inhibitors specific to RhoA signaling or the signaling that suppresses RhoA activation, such as Src family kinases and ERK-MAPK signaling, promote vessel stability. ${ }^{19-21}$

Rho family GTPases and cadherin-mediated cell-cell adhesion are mutually regulated. ${ }^{25-28}$ For example, RhoA-dependent cytoskeletal tension promotes the disassembly of cell-cell adhesion via myosin II-mediated actin reorganization. ${ }^{29}$ Expression of constitutively activated RhoA disrupts adherens junctions, and inhibitors of RhoA signaling reinforce cell-cell adhesions. ${ }^{26}$ On the other hand, the adherens junctional complex consisting of cadherin, $\beta$-catenin, and $\mathrm{p} 120^{\mathrm{ctn}}$ inhibits RhoA signaling through recruiting p190Rho ${ }^{\mathrm{GAP}}$ into the junctional complex. ${ }^{30}$ Hence, RhoA likely balances cytoskeletal tension and cell-cell adhesion.

\subsection{Tetraspanins and Vascular Functions}

Tetraspanins are a group of type III transmembrane proteins which span cell membrane 4 times. ${ }^{31}$ Tetraspanins contain four conserved hydrophobic transmembrane domains (TM1-TM4), intracellular N- and C-terminal domains, and small (EC1) and large (EC2) extracellular domains. ${ }^{31-35} \mathrm{~A}$ schematic representation of tetraspanin domain structure is shown in Figure 1-1. The EC2 domain of tetraspanins features several 


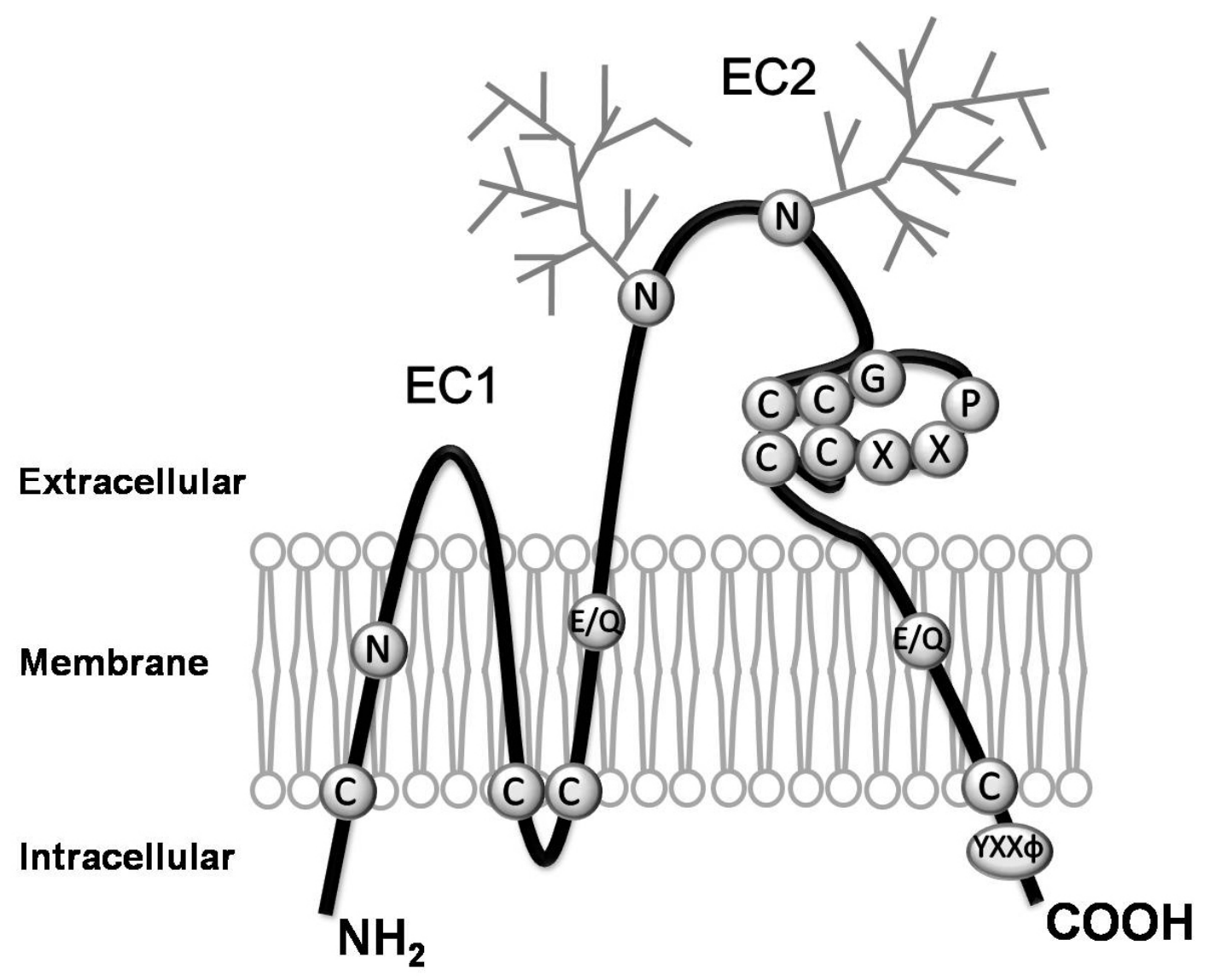

Figure 1-1. Schematic representation of the structure of tetraspanins.

Tetraspanins consist of four transmembrane, intracellular N- and C-terminal domains, and two extracellular (one shorter, EC1, and one longer, EC2) domains. The EC2 is featured by the 'CCG' and 'PXXCC' motifs. Most of tetraspanins have glycosylation sites in EC2 as indicated as the squares, while CD9 is glycosylated in EC1. In each tetraspanin, there are several cysteine residues proximal to the interface of the inner leaflet and cytosol, which are the sites for palmitoylation and characteristic strong polar residues in the transmembrane domains. Tetraspanins also contain a ' $\mathrm{XX \Phi}$ ' endocytic sorting motif in the C-terminal cytoplasmic domain. C, Cysteine; E, Glutamic acid; G, Glycine; P, Proline; Q, Glutamine; N, Aspargine; S, Serine; Y, Tyrosine; X, any amino acid; $\Phi$, Hydrophobic amino acid. 
cysteine residues, glycosylation sites, and highly conserved "CCG" and "PXXCC" motifs, while transmembrane domains contain palmitoylation sites. Tetraspanins interact with each others and with other membrane bound proteins such as integrins ${ }^{31-35}$ and Ig superfamily proteins ${ }^{36}$ through numerous protein associations to form tetraspanin-containing multimolecular complexes in the membrane designated as "tetraspanin web" or "tetraspanin enriched microdomains" (TEM). ${ }^{31,37,38}$ TEMs are organized as the functional units for specific regulations of various cellular processes such as cell adhesion, enzymatic activities, protein compartmentalization and membrane presentation and etc on cell plasma membrane. ${ }^{31,37,38}$ However, with the exception of CD151-integrin $\alpha 3 \beta 1$ binding, most interactions of tetraspanins with other transmembrane proteins are probably not based on the direct amino acid residue-amino acid residue interactions at the EC regions because they are readily disrupted under the so called "high stringency" detergent conditions used to lyse cells (e.g., 1\% Triton X-100).

As the organizers in TEM, tetraspanins regulate vital cellular processes such as adhesion, spreading, migration, and fusion and these processes-dependent signaling pathways (Figure 1-2) as observed in various in vitro and in vivo systems. ${ }^{39-43}$ In addition, recent studies reveal that tetraspanins actively traffic between plasma membrane and intracellular vesicular compartments, and their trafficking is accompanied by the vesicular release and regulates the trafficking of other membrane proteins such as integrins. ${ }^{44,45}$ Another emerging theme is that tetraspanins appear to regulate pericellular proteolysis near the plasma membrane, leading to altered cell movement and adhesiveness. $^{46-49}$ Thus, tetraspanins have been defined as molecular "facilitators" or "organizers" at the plasma membrane of both intracellular vesicles and extracellular microenvironment.

Many tetraspanins are expressed in vascular system. For example, CD9, CD63, and CD151 are highly expressed in endothelial cells, and others such as TSSC6 are expressed in hematopoietic cells. Because cell adhesion proteins regulate vascular events such as neointimal formation, angiogenesis, and thrombosis and tetraspanins play key roles in adhesion and migration, tetraspanins likely modulate important pathophysiological process of the vascular system. Indeed, by differentially associating with integrins and other membrane molecules, tetraspanins such as CD9, CD151, CD63 and TSSC6 take part in diverse vascular events including smooth muscle cell (SMC) proliferation and migration, endothelial cell adhesion, platelet aggregation and thrombosis, angiogenesis, and haemostasis. ${ }^{50}$ The biology of vascular tetraspanins and their regulatory roles in the pathophysiological processes related to cardiovascular system become better understood recently. ${ }^{50}$ However, the expression and function of most newly identified tetraspanins in the vascular system remain to be elucidated. 


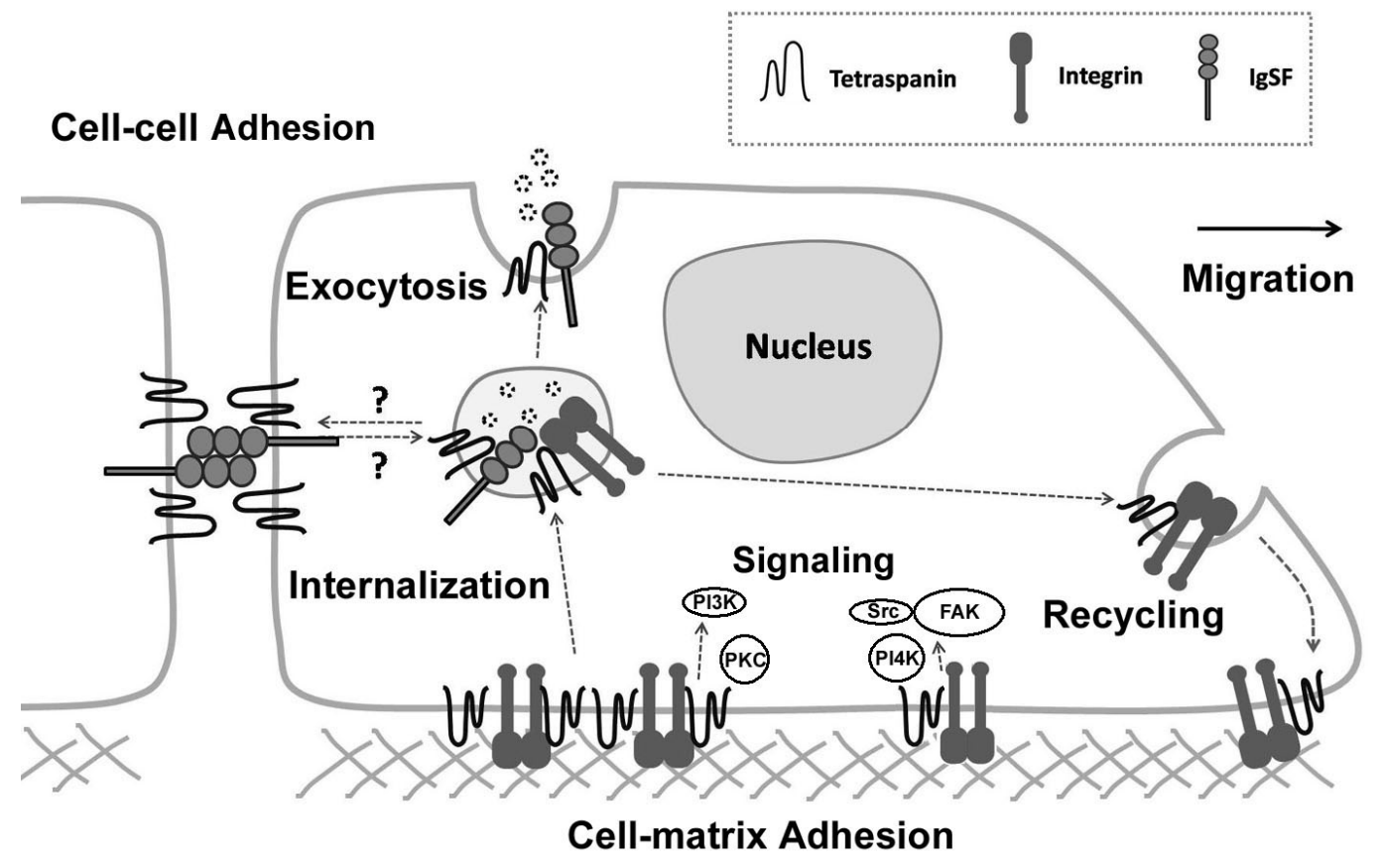

Figure 1-2. Schematic drawing showing cellular functions of tetraspanins.

Tetraspanins associate with integrins, Ig superfamily proteins, and other transmembrane proteins to form microdomains on membrane designated as 'tetraspanin web' or 'tetraspanin enriched microdomians' (TEM) and serve as organizers at the plasma membrane. Tetraspanins are also enriched in the intracellular vesicles, and trafficking of tetraspanins between various cellular compartments tightly regulates exocytosis and trafficking of their associated partners. Tetraspanins, in conjunction with other membrane proteins, regulate cell-matrix adhesion, cell-cell adhesion, cell migration, and intracellular signaling events. 


\subsection{Tetraspanin CD151}

\subsubsection{Chromosomal Locus of CD151 Gene}

CD151, originally identified as platelet-endothelial cell tetra-span antigen 3 (PETA-3), is a member of tetraspanin family. ${ }^{51}$ Human CD151 gene is located on chromosome $11 \mathrm{p} 15.5 .^{52}$ The gene spans $\sim 5 \mathrm{~kb}$ of genomic DNA and consists of 8 exons. ${ }^{52}$ Mouse $C D 151$ gene spans $\sim 4 \mathrm{~kb}$ on chromosome 7 , and comprises 7 exons. ${ }^{53,54}$ The exon-intron organization of human $C D 151$ gene is summarized in Figure 1-3A. ${ }^{52,54}$

\subsubsection{Gene Expression and Regulation of CD151}

Human CD151 gene produces two transcripts by alternative splicing and forms $1.6 \mathrm{~kb}$ mRNA molecules. The shorter transcript variant PETA-3 has a 62-bp deleted region in the 5 ' -untranslated region compared with the longer one. ${ }^{55}$ Mouse CD151 mRNA is approximately $1.8 \mathrm{~kb}$ in length, and has three alternative splicing variants with different 5'-untranslated region regions. ${ }^{53,54}$ Both human and mouse CD151 mRNAs contain a single open reading frame encoding 253 amino acid residues with $93 \%$ identity to each other. ${ }^{53}$

A recent study demonstrated that the transcription factor SP1 controls CD151 expression in human liver cancer cells partially through establishing a local open chromatin configuration in CD151 promoter. ${ }^{56} \mathrm{CD} 151$ expression can also be induced by human T-cell leukemia virus type 1 (HTLV-1) and transactivated by Tax in platelets. ${ }^{57}$ In addition, CD151 expression can be upregulated after overexpression of its associated integrin $\alpha 3 \beta 1, \alpha 6 \beta 4$ or $\alpha 7 \beta 1$ in cultured cells such as K562 cells and PA-JEB keratinocytes through mechanisms that remain unclear. ${ }^{58,59}$

\subsubsection{Protein Structure of CD151}

CD151 protein consists of 253 amino acid residues with a molecular weight of $\sim 28 \mathrm{kDa}^{51}$ As a member of tetraspanin family, CD151 has a typical tetraspanin domain structure, containing four transmembrane domains (TM1-TM4), a short and long extracellular domains (EC1 and EC2), and N- and C-terminal cytoplasmic domains (Figure 1-3B). ${ }^{51-53}$ In human CD151, each of the four transmembarne domains is formed by a hydrophobic segment containing $\geq 22$ amino acid residues. ${ }^{51}$ The hydrophilic EC1 (between TM1 and TM2) and EC2 (between TM3 and TM4) domains are composed of 18 (positions 40-57) and 109 (positions 113-221) residues, respectively, while the 


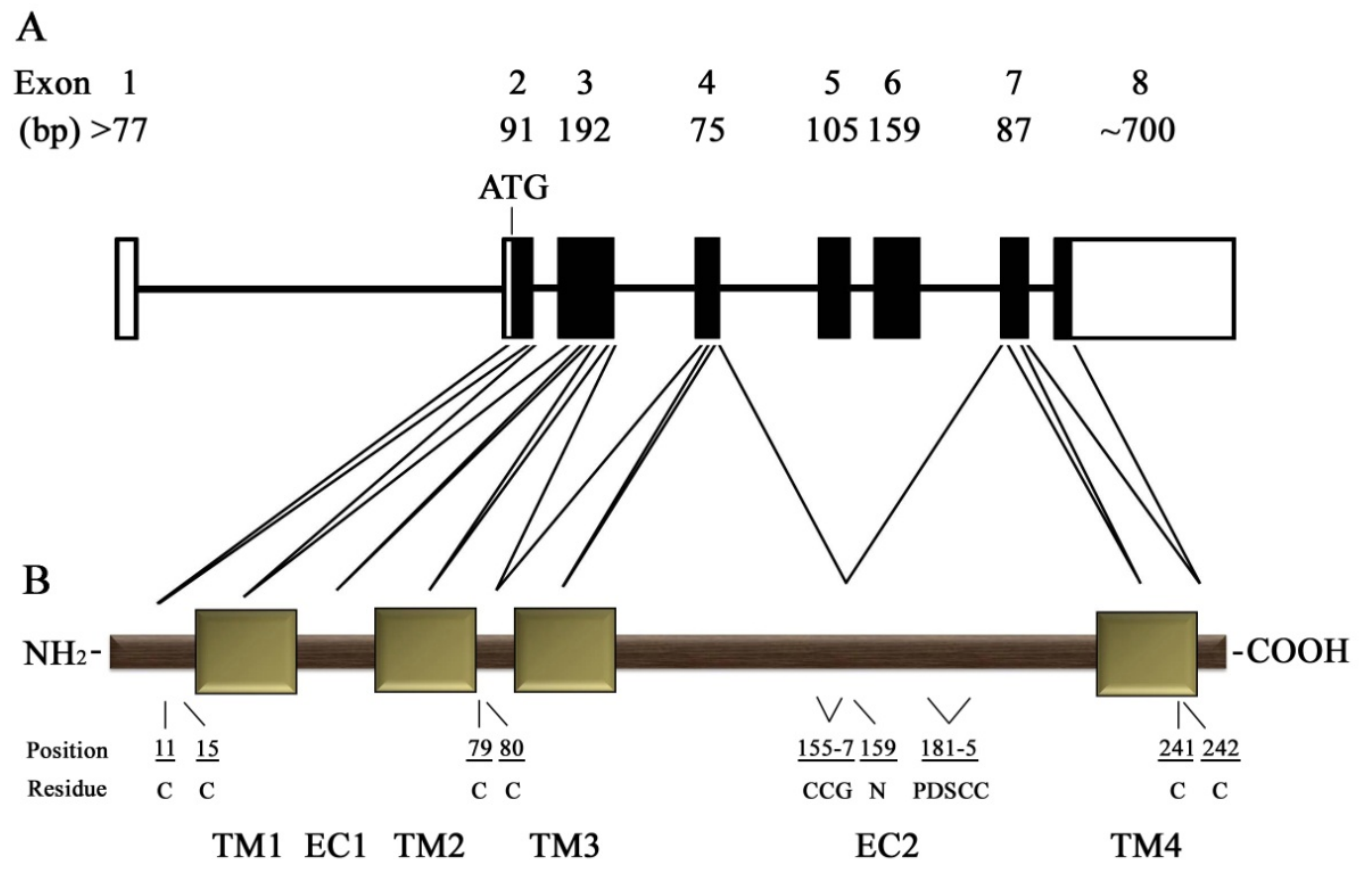

Figure 1-3. Schematic drawing of genomic organization and protein domain structure of human CD151.

(A) Intron-exon organization of CD151. CD151 gene contains 8 exons, 75-700bp in size, with 5'- and 3'-noncoding regions $(\square)$ and coding region ( $\square$ ). (B) Protein domain structure of CD151. The protein has a typical tetraspanin domain structure (Figure 1-1) consisting of N- and C-terminus, TM1-TM4, EC2 and EC2 domains with featured residues. The corresponding exon coding regions of the domains are indicated. 
intracellular N- and C-termini consist of 16 (positions 1-16) and 7 (positions 247-253) hydrophilic residues, respectively. ${ }^{51,60}$ The EC2 contains six cysteine residues, four of which are located in "CCG" and "PDSCC" motifs corresponding to residues 155-157 and 181-185, respectively. ${ }^{51}$ Both cysteine-containing motifs are critical in determining appropriate folding of the EC2 domain of $\mathrm{CD} 151{ }^{61}$ A potential glycosylation site, located at asparagine residue 159, appears not to be required for interactions of CD151 with other tetraspanins but rather contributes to the modulatory activities of CD151. ${ }^{62} \mathrm{In}$ addition, like other tetraspanins, CD151 contains a set of palmitoylation sites at the cysteine residues, including positions 11, 15, 79, 80, 241 and 242, proximal to TM1, TM3 or TM4 ${ }^{63,64}$ The palmitoylation of CD151 occurs in Golgi or post-Golgi compartments, ${ }^{64}$ and is specifically mediated by DHHC2, a member of the "DHHC" (Asp-His-His-Cys) family of protein acyltransferases (PATs). ${ }^{65}$ It has been shown that the palmitoylation protects CD151 from lysosomal degradation and is important in establishing the interactions of CD151 with integrins and other tetraspanins in TEM and modulating integrin-dependent functions. ${ }^{63-66}$ Notably, because of the glycosylation and palmitoylation of the protein, the appearance of CD151 on SDS-PAGE is a diffuse band with a range of $27-37 \mathrm{kDa}$ in molecular weight.

\subsubsection{Tissue Localization of CD151}

CD151 is ubiquitously expressed. Northern blotting analysis showed that CD151 mRNA is present in all tissues, with the exception of brain, in both human and mouse. ${ }^{51,53}$ Immunohistochemistry analysis confirmed the broad distribution of CD151 protein in human tissues ${ }^{67}$ With respect to cell types, CD151 is expressed by endothelial cells, epithelial cells, fibroblasts, platelets, megakaryocytes, erythrocytes, Schwann cells, dendritic cells and by all types of muscle cells. ${ }^{67,68}$ The expression of CD151 in endothelial cells is particularly high and throughout vasculature of all human tissues, ${ }^{67}$ suggesting it plays important roles in vascular functions.

\subsubsection{Association of CD151 with Integrins and Other Tetraspanins}

Previous studies have well established that CD151 interacts with laminin-binding integrins $\alpha 3 \beta 1, \alpha 6 \beta 1, \alpha 6 \beta 4$, and $\alpha 7 \beta 1$ and other tetraspanins such as CD9, CD81 and CD63. ${ }^{59-61,63,64,69-73} \mathrm{CD} 151$ associates with at least one of the laminin-binding integrins in epithelial and endothelial cells and myocytes, and the association is independent of the ligand occupancy of these integrins. Whereas it may associate other integrins in fibroblasts or stromal cells which have no laminin-binding integrins present. ${ }^{71,74}$

CD151 selectively forms highly stable and stoichiometric associations with 
integrin $\alpha 3 \beta 1$, which shows resistance to the extraction by high stringency detergent (e.g., $1 \%$ Triton $\mathrm{X}-100){ }^{59,69}$ The interaction of CD151 with integrin $\alpha 3 \beta 1$ is mediated directly by physical contact between EC2 region of CD151 and the membrane-proximal region of integrin $\alpha 3$ ectodomain. ${ }^{59,61}$ The mutagenesis analysis suggests that the region containing residues 150-212 in EC2 of CD151 confers the specific and strong interaction of CD151 with integrin $\alpha 3 \beta 1$, whereas EC1 and TMs are responsible for surface localization of CD151. ${ }^{61}$ The sub-region of residues $185-217$ in EC2 is of particular importance in CD151-integrin $\alpha 3 \beta 1$ association. ${ }^{60,61}$ Results from epitope mapping with CD151 mAbs have confirmed that two consecutive segments within the above specific 185-217 sub-region, the Leu191-containing 185-192 peptide and the Gln194-containing QRD peptide, comprise an interface between CD151 and integrin $\alpha 3 \beta 1$ and mediate the stable association between the two proteins, although other residues such as Gly176/Gly177 and the cysteine residues in "CCG" and "PDSCC" motifs are also important for CD151/integrin $\alpha 3 \beta 1$ interaction. ${ }^{61,75}$ In contrast to the interactions of CD151 with other integrins and tetraspanins, the one between CD151 and integrin $\alpha 3 \beta 1$ exhibits a unique feature, i.e. the assembly of integrin $\alpha 3 \beta 1-C D 151$ complex occurs before their secretion onto plasma membrane, likely during early stage of the biosynthesis of the integrin in ER. ${ }^{61}$ The interactions between CD151 and integrin $\alpha 6 \beta 1$ and $\alpha 6 \beta 4$ are weaker so that it is not resistant to harsh detergents such as 1\% Nonidet P-40 (NP-40) but still fairly stable, ${ }^{58,71,73,76}$ while the association of integrin $\alpha 7 \beta 1$ with CD151 is equally strong compared with that of $\alpha 3 \beta 1 .^{71} \mathrm{CD} 151$ may also use EC2 to interact with the $\alpha$ subunit in integrins $\alpha 6 \beta 1, \alpha 6 \beta 4$, and $\alpha 7 \beta 1 .^{58,59,71,76}$ However, the structural elements in CD151 responsible for associations with these integrins need to be further elucidated.

CD151 also interacts with other tetraspanins such as CD9, CD81, and CD63 and thus participates in the formation of TEM. ${ }^{61,64,77,78}$ These interactions appear to be indirect and less stable. ${ }^{61,78}$ Mutagenesis analysis suggests the EC2 region is not required for the interactions of CD151 with CD9, CD81, and CD63, while the palmitoylation of CD151 is necessary for these interactions. ${ }^{61,64,66}$ In addition to the heterotypic interaction with other tetraspanins, CD151, like other tetraspanins, also forms homodimers which appear to be more stoichiometric and prevalent than the heterodimers such as CD9/CD151 and CD81/CD151. ${ }^{61,78}$ These homodimers are believed to be assembled from newly synthesized proteins in Golgi and subsequently transported to the cell surface where they might serve as so called "building blocks" for establishment of "tetraspanin web" with other proteins. ${ }^{61,78}$ The cysteine residues at the palmitoylation sites and in the "CCG" motif of CD151 play pivotal roles in the assembly of the dimmers. ${ }^{61,78}$ 


\subsubsection{Functions of CD151}

\subsubsection{The Roles of CD151 in Integrin-dependent Functions}

CD151 is believed to be an important adaptor which facilitate connection of integrins to the tetraspanin web and thus modulate integrin functions. ${ }^{59} \mathrm{CD} 151$ is constantly involved in integrin $\alpha 3 \beta 1$-dependent processes due to their firm association. First, CD151 regulates $\alpha 3 \beta 1$-dependent cell motility in a variety of cells. ${ }^{59,79,80}$ Second, CD151 colocalizes with integrin $\alpha 3 \beta 1$ in cell-matrix adhesion structures and substantially modulates $\alpha 3 \beta 1$-dependent cell adhesion on not only laminin 5 but also laminin 10 , the two ligands of $\alpha 3 \beta 1$, likely through potentiating the ligand-binding abilities of the integrin by stabilizing its active conformation. ${ }^{79,81-83}$ Moreover, integrin $\alpha 3 \beta 1-C D 151$ complex acts as a functional unit and makes a strong contribution to cell morphogenesis and neurite outgrowth. ${ }^{82,84}$ Mechanistically, CD151 appears to mediate compartmentalization of $\alpha 3 \beta 1$ into $\mathrm{TEM}^{44,63,79}$ and associate with and/or modulate major signaling molecules such as the phosphatidylinositol signaling effectors phosphatidylinositol 3 kinase (PI3K) ${ }^{63}$ and phosphatidylinositol 4 kinase (PI4K), ${ }^{59}$ protein kinase C (PKC), ${ }^{85,86}$ and receptor tyrosine kinase cMet/HGF receptor. ${ }^{87} \mathrm{CD} 151$ thus selectively regulates these pathways and trigger the downstream signaling events such as protein phosphorylation for Src, focal adhesion kinase (FAK), p130Cas, paxillin, and $\alpha 3$ integrin, through which CD151 contributes to $\alpha 3 \beta 1$-dependent cell proliferation, migration, adhesion, and morphogenesis. ${ }^{59,63,79,82,85-90}$

Integrin $\alpha 6 \beta 4$ is another cellular receptor of laminin $5{ }^{58}$ Similarly, CD151 contributes to integrin $\alpha 6 \beta 4$-dependent processes such as cellular morphogenesis and the spatial organization of hemidesmosomes possibly by enforcing integrin $\alpha 6 \beta 4$ connection to the tetraspanin web. ${ }^{58,64} \mathrm{CD} 151$ has also been shown to selectively promote integrin $\alpha 6 \beta 1$-mediated cell spreading and adhesion strengthening on laminin 1 (an integrin $\alpha 6 \beta 1$ ligand) and cellular morphogenesis on Matrigel. ${ }^{76,91}$ The short C-terminal cytoplasmic domain of CD151 is believed to be of particular importance for modulating "outside-in" functions of integrin $\alpha 6 \beta 1$ upon ligand binding. ${ }^{76,91}$ Although the functional relationship between CD151 and inegrin $\alpha 7 \beta 1$, a receptor of laminin 2, is still unclear, it is predicted that CD151 may participate in integrin $\alpha 7 \beta 1$-dependent functions such as Schwann cell myelination and muscle development. ${ }^{92-95}$

The concept of tetraspanins as adaptors mediating compartmentalization of integrins into TEM and organizing the interactions of integrins with other proteins within TEM helps explain how tetraspanins facilitate integrin functions. How does CD151 mediate compartmentalization of integrins? On the one hand, CD151 specifically modulates the post-translational modifications of its associated integrins such as the 
glycosylation of integrin $\alpha 3 \beta 1$ on $N$-linked sites of the $\alpha 3$ subunit during the biosynthesis of this integrin in ER and Golgi. ${ }^{62}$ Such modifications likely affect the stability, folding, and membrane presentation of this integrin. ${ }^{62}$ On the other hand, CD151 directly regulates integrin trafficking to and from the plasma membrane and between intracellular compartments. CD151 and its associated integrins undergo endocytosis and accumulate in the same intracellular vesicular compartments, suggesting a key role for CD151 in regulating the trafficking of these integrins. ${ }^{44,73}$ Indeed, during cell migration on laminin 1 or 5, CD151 promotes the endocytosis and/or vesicular trafficking of integrins $\alpha 3 \beta 1$, $\alpha 5 \beta 1$, and $\alpha 6 \beta 1 .^{44,79}$ It has been demonstrated that a YXXФ type of endocytosis/sorting motif, the YRSL sequence, is located at the C-terminal cytoplasmic domain of CD $151 .{ }^{44}$ The YXXФ motif-mediated internalization of CD151 plays critical roles in modulating integrin trafficking and thereby contributes to integrin-dependent cell migration. ${ }^{44}$

\subsubsection{CD151 Is a Tumor Metastasis Promoter}

Increased CD151 expression has been shown to be associated with a poor prognosis for patients with lung, liver, prostate cancer or other cancer types. ${ }^{96-100}$ Given the ability of CD151 to promote cell migration, earlier studies have identified CD151 as a positive regulator for tumor metastasis. ${ }^{98,101-104}$ Tumor metastasis is the process by which tumor cells migrate from primary tumor to distal organs and represents advanced malignancy. ${ }^{105,106}$ The metastasis cascade contains numerous sequential biological processes which can be in general subdivided into the following steps: 1) cell dissociation from primary tumor foci; 2) cell migration and invasion and angiogenesis; 3 ) intravasation; 4) circulation transportation; 5) extravasation; 6) formation of metastatic foci; and subsequent 7) tumor cell proliferation and angiogenesis. ${ }^{105-107}$ Among these steps, CD151 specifically takes part in tumor cell migration and invasion, intravasation, extravasation, and angiogenesis by altering cell motility, cell adhesion, proteolytic activity of MMPs, and transforming growth factor $\beta$ (TGF $\beta$ ) signaling.

Cell motility is a key parameter that determines the behavior of malignant tumor cells throughout the entire metastatic process. Integrin-dependent cell migration is apparently crucial for tumor metastasis, and CD151 enhances integrin-dependent migration and invasion of a wide variety of tumor cells in vitro. ${ }^{72,79,87,101,104,108-110}$ In vivo, CD151 promotes tumor metastasis by enhancing tumor cell motility. ${ }^{101,102,104}$ CD151 was shown to enhance tumor cell migration during their departure from primary tumor mass, which leads to tumor cell invasion into surrounding connective tissue and intravasation. ${ }^{101,102}$ A metastasis-blocking CD151 mAb (IA5) was found to immobilize tumor cells by blocking intravasation and subsequently suppress metastasis in vivo. ${ }^{102}$ The regulatory role of CD151 in cell migration is apparently dependent on its association with laminin-binding integrins. ${ }^{44,59,79,104}$ Mechanistically, migrating cells form protrusions 
such as lamellipodia and filopodia containing motile structures which mediate the detachment and re-attachment cycle for migration and induce small GTPases Rac- and Cdc42- dependent reorganization of actin cytoskeleton. ${ }^{111} \mathrm{CD} 151$ was found to 1) induce Rac1 and Cdc42 signaling and actin cytoskeletal reorganization, ${ }^{74,85,112}$ 2) colocalize with integrin $\alpha 3 \beta 1$ at the leading edges of lamellipodia and filopodia, ${ }^{79,81}$ and 3) regulate integrin $\alpha 3 \beta 1$-initiated signaling to FAK and PI4K, which are important regulators in cell adhesion and cytoskeleton reorganization, respectively. ${ }^{59,82,112}$ Thus, to enhance cell motility, CD151-integrin $\alpha 3 \beta 1$ complexes induces lamellipodia and filopodia formation and directly modulates the dynamic adhesive structures at the edge of these cellular extensions. However, other mechanisms by which CD151 regulates cell motility also exist. For example, CD151 promotes cell migration by regulating intracellular trafficking of integrins. ${ }^{44,79}$ It has been proposed that, during cell migration, the internalization of CD151 result in the endocytosis of its associated TEM components such as integrin $\alpha 3 \beta 1$ when this integrin disengages from ECM. ${ }^{44}$ The ECM-disengaged integrins are internalized from the trailing edge of migrating cells and likely recycled back to the leading edge under CD151 escort to re-engage with ECM. ${ }^{44}$ In this regard, CD151 enhances the internalization and reutilization of integrins by facilitating their trafficking and thus accelerates the detachment and re-attachment cycles to enhance cell migration.

Another aspect of tumor metastasis that CD151 may affect is cell adhesion, both cell-matrix adhesion and cell-cell adhesion, within primary tumor and during metastasis. Cell-matrix adhesion is believed to be the cell behavior crucial for tumor metastasis not only because of the importance of cell adhesion in tumor cell motility, as discussed above, but also because of the adhesion-dependent activation of various metastasis-required signaling events such as MMPs production and protein phosphorylation. ${ }^{46,49,59,63,86,113-115}$ It has been extensively discussed that the principal role of CD151 is to stabilize cell-matrix interaction by associating with integrins $\alpha 3 \beta 1$ and $\alpha 6 \beta 4$ at the basal cell surface. ${ }^{58,79,81-83}$ Thus, it is plausible that CD151-mediated promotion of tumor metastasis is largely dependent on its ability to facilitate integrins to establish adhesion of metastatic cells to ECM during not only tumor cell migration and invasion but also in the subsequent formation of metastatic foci in distal organs. In addition to cell-matrix adhesion, CD151 has also been shown to stabilize the homotypic cell-cell adhesion of epithelial or tumor cells ${ }^{85,116,117}$ and heterotypic adhesion between endothelial cells and leukocytes during leukocyte extravasation. ${ }^{118} \mathrm{~A}$ substantial proportion of cell surface CD151 colocalizes with integrin $\alpha 3 \beta 1$ and cadherins at lateral junctional sites in both epithelial and endothelial cells, ${ }^{73,85,108,116,117}$ suggesting a role of CD151-integrin $\alpha 3 \beta 1$ complex in regulation of cadherin-based AJs. The establishment of AJ involves cell polarization, fillipodia-based membrane extension and contact, cadherin puncta formation and assembly of the junctional complex, and anchorage of the complex to actin cytoskeleton through the linkers such as $\alpha$-actinin and vinculin. ${ }^{119,120}$ Observations from epithelial and tumor cells suggest that CD151-integrin $\alpha 3 \beta 1$ complex 
is associated with AJ and essential for the filopodia-based adhesion zipper formation at an initial stage and the anchorage of E-cadherin to cortical actin filaments at later stages in a PKC- and Cdc42-dependent manner, without affecting AJ complex assembly. ${ }^{85,116,117}$ The downregulation of E-cadherin is the hallmark of eptithelial-mesenchymal transition (EMT), which may lead to tumor metastasis under pathological conditions. ${ }^{121}$ CD151-dependent regulation of cell-cell adhesion in epithelial and tumor cells might provide another potential mechanism by which CD151 facilitates tumor progression. Moreover, CD151 in its associated TEM has a crucial role in ICAM-1 and VCAM-1-mediated heterotypic intercellular adhesion between endothelial cells and leukocytes during leukocyte extravasation. ${ }^{118}$ The CD151- or CD9-containing TEMs include ICAM-1 and VCAM-1 and regulate the membrane distribution and adhesion activity of ICAM-1 and VCAM-1, thereby promoting leukocyte-endothelium adhesion and leukocyte tranendothelial migration, the essential steps of leukocyte extravasation. ${ }^{118}$

During tumor metastasis, the local invasion and infiltration of primary tumor, tumor-induced angiogenesis, and establishment of metastatic foci involve degradation of the ECM by matrix metalloproteinases (MMPs), the well-established master regulators of angiogenesis and tumor progression. ${ }^{122,123} \mathrm{CD} 151$ has been shown to modulate expression and activation of several MMPs including MMP-2, MMP-7, MMP-9, and MT-MMP in cancer and endothelial cells. ${ }^{46,49,113-115}$ In hepatocellular carcinoma (HCC) patients, high CD151 expression was found to be correlated with elevated expression of MMP-9, microvessel density (MVD), poor overall survival rate and high cumulative recurrence after surgery. ${ }^{98}$ Signal transduction analysis suggests that CD151 induces the secretion of MMP-9 via an adhesion-dependent c-Jun signaling pathway involving either the activation of FAK, Src, p38 MAPK, and JNK or the PI3K/Akt/GSK-3 $\beta /$ Snail pathway in tumor cells, leading to increased tumor cell invasiveness and angiogenesis, respectively. ${ }^{98,110}$ The modulatory activity of CD151 toward MMP-9 seems to be dependent on both integrin-mediated cell-matrix adhesion and homophilic interactions of CD151 proteins with neighboring cells. ${ }^{98,110}$ It was also found that CD151-integrin $\alpha 3 \beta 1$ complex induces MMP-2 production in breast cancer and epidermal carcinoma cells. ${ }^{46,115}$ Moreover, in human rectal carcinoma cells and osteoarthritic chondrocytes, CD151 colocalizes with proMMP-7 at the plasma membrane where it physically binds to the protein through the EC2 domain and induces pericellular activation of proMMP-7. ${ }^{113,114}$ Another study demonstrated that CD151 regulates MMP-7 expression and co-distributes with MMP-7 at the leading edge of lamellipodia of migrating epidermal carcinoma cells. ${ }^{115}$ These findings, together with the fact that MMP7 is expressed preferentially at invasive edges in tumors, suggest that $\mathrm{CD} 151$ promotes the progression of tumors as well as other disease such as osteoarthritis by MMP-7-dependent invasiveness and proteolytic activity. ${ }^{113-115}$ Endothelial CD151 was also found to associate with MT1-MMP, link MT1-MMP to integrin $\alpha 3 \beta 1$ to form the integrin $\alpha 3 \beta 1 / C D 151 / M T 1-M M P$ ternary complexes, and modulate the trafficking and enzymatic activity of this 
membrane-anchored proteinase, implying that CD151 is a key regulator for endothelial homeostasis and angiogenesis. ${ }^{49}$

TGF $\beta$ signaling is an important pathway in modulation of EMT, a process characterized by loss of cell adhesion, rearrangement of actin cytoskeleton, repression of E-cadherin expression, and increase of cell mobility. TGF $\beta$ signaling is also required for both physiological processes, such as embryogenesis and wound healing, and pathological processes, such as tumor procession. ${ }^{121}$ Upon binding and activating its receptors, TGF $\beta 1$ triggers downstream signaling by inducing the formation and activation of a Smad2/3/4 transcriptional complex with other co-regulators and thus initiates re-epithelialization. Smad7 blocks this signaling pathway by competing with $\mathrm{Smad} 2$ and Smad3 to bind TGF $\beta 1$ receptors. ${ }^{124}$ Integrin $\alpha 3 \beta 1$ has been recently shown to regulate TGF $\beta 1$ signaling through TGF $\beta 1$ downstream effector Smad 2 and inhibitor Smad7 and be required for initiation of re-epithelialization and EMT during wound healing and pulmonary fibrosis in mice. ${ }^{125,126}$ Integrin $\alpha 3 \beta 1$ could be involved in the Smad signaling by either mediating the formation of Smad 2 transcriptional complex with $\beta$-catenin after phosphorylation or blocking the inhibitory activity of Smad7. ${ }^{125,126}$ Given the close functional relevance between integrin $\alpha 3 \beta 1$ and CD151, CD151 might also modulate TGF $\beta 1$ signaling-induced EMT during tumor procession. This hypothesis is supported several lines of evidence. First, loss of CD151 has been shown to result in deficient wound healing in mice, characterized by retarded re-epithelialization and wound closure, suggesting that CD151 plays similar roles in tumor EMT. In fact, a recent study revealed that CD151 functions as a regulator in TGF $\beta 1$-induced breast cancer progression. ${ }^{98}$ Loss of CD151 attenuates TGF $\beta 1$-induced scattering and proliferation of breast cancer cells in 3D Matrigel and correlates with reduced retention of cancer cells in lung vascular bed and decreased metastasis to lungs. ${ }^{98}$ However, CD151-dependent regulation in these processes appears not to involve the activation of $\operatorname{Smad} 2 / 3$ but rather the

compartmentalization of TGF $\beta$ type I receptor (TGF $\beta$ R I) and activation of p38 MAPK. ${ }^{98}$ Thus, the mechanistic connection of CD151 to TGF $\beta 1$-induced EMT is still elusive. Future studies on the relationship between CD151/integrin $\alpha 3 \beta 1$ and TGF $\beta 1 / T G F \beta R /$ Smads will be necessary to thoroughly elucidate the roles of CD151 in tumor procession.

\subsubsection{CD151 in Hematopoietic Cells}

In hematopoietic cells, CD151 expression is mostly restricted to platelets, megakaryocytes, erythrocytes, dendritic cells, and activated T lymphocytes. ${ }^{67,68,70}$ Because most hematopoietic cells do not express laminin-binding integrins, the functions of CD151 in these cells could be independent of laminin-binding integrins. 
A C-terminal deletion mutation of CD151 in humans leads to severe defects in erythropoiesis, ${ }^{127}$ underlining a connection between CD151 and proper functioning of hematopoietic cells. However, CD151-null mice are normal in terms of the development of hematopoietic cells and proliferation of T lymphocytes. ${ }^{128-130} \mathrm{CD} 151$ also appears to modulate adhesion of hematopoietic cells such as the homotypic cell-cell adhesion of erythroleukemia and megakaryoblastic cells. ${ }^{70}$

CD151 plays important roles in platelet functions. It has been demonstrated that CD151 is essential for platelet spreading on fibronectin, platelet aggregation responses to protease-activated receptor 4 (PAR-4) agonist peptide, collagen, and adenosine diphosphate (ADP), and platelet thrombus formation and stabilization after $\mathrm{FeCl}_{3}$-induced injury of mesenteric arterioles in mice. ${ }^{129,131} \mathrm{CD} 151$ physically associates with integrin $\alpha \operatorname{IIb} \beta 3$, the major integrin in platelets, and is involved in regulation of integrin $\alpha \operatorname{IIb} \beta 3$ "outside-in" signaling, which supports the notion that CD151 acts as a modulator of integrin $\alpha \operatorname{IIb} \beta 3$ signaling and thus contributes to platelet functions. ${ }^{129}$

In human T lymphocytes, CD151 expression is trans-activated by Tax in response to human $\mathrm{T}$ cell leukemia virus type 1 (HTLV-1) infection and promotes integrin $\alpha 5 \beta 1$-dependent adhesion to fibronectin in HTLV-1-positive T cells. ${ }^{132,133}$ Moreover, observations from an in vitro $\mathrm{T}$ cell stimulation assay suggest that CD151 is important for antigen presentation by dendritic cells (DC) to T cells. ${ }^{134} \mathrm{CD} 151$ was found to specifically regulate the co-stimulation abilities of DC during antigen presentation to CD4+ and CD8+ T cells without affecting DC development or maturation, suggesting a regulatory activity of CD151 toward antigen (Ag)-specific cellular immune responses. ${ }^{134}$ In addition, CD151 may also play a role in the entry of human immunodeficiency virus type 1 (HIV-1) into macrophages. Recombinant EC2 domains of CD151, as well as tetraspanins CD9, CD63, and CD81, are capable of inhibiting cellular uptake of HIV-1 virions perhaps by altering the organization of CD4-HIV complexes within the tetraspanin web, which are required for membrane fusion events. ${ }^{135}$

\subsubsection{CD151 in Vascular Endothelium and Its Role in Angiogenesis}

In human umbilical vein endothelial cells (HUVECs), intracellular CD151 accounts for up to $66 \%$ of the total CD151 and resides in endosomal/lyosomal vesicles, while CD151 at the cell surface is localized at cell-cell junctions together with CD9 and CD81, the primary tetraspanins that are associated with CD151. ${ }^{73,80}$ The

CD151-containing TEM on endothelial cells is critical for proper function of endothelial cell-cell adhesion proteins such as ICAM-1 and VCAM-1. ${ }^{118}$ Endothelial cell surface CD151 is needed for the transendothelial migration of lymphocytes by strengthening the firm adhesion of lymphocytes to endothelial cells during extravasation. ${ }^{118}$ In addition, 
CD151-integrin complex regulates endothelial cell migration. ${ }^{73,74,80}$

Accumulating evidence indicates that CD151 is an important regulator in vascular morphogenesis. First, CD151 supports the vascular morphogenetic process that endothelial cell undergo on/in 3-dimensional matrix. ${ }^{73,74,76}$ Second, CD151 overexpression in heart after myocardial infarction substantially increases the myocardial microvessel density, suggesting that CD151 promotes neovascularization. ${ }^{89}$ Third, CD151 knockout mice are deficient in pathologic angiogenesis, based on the observations from in vivo Matrigel plug, corneal micropocket, and tumor implantation angiogenesis assays as well as ex vivo aortic ring angiogenesis assay. ${ }^{74}$ Fourth, CD151 modulates the expression and enzymatic activity of MMP-9 and MT1-MMP in cancer and endothelial cells. $^{49,98,122}$ Because MMP-9 and MT1-MMP are pro-angiogenic, this result supports the conclusion that CD151 promotes tumor angiogenesis.

However, the mechanisms by which CD151 regulates vascular morphogenesis remain largely unknown. Mechanistic studies from epithelial and tumor cells indicate that CD151, through its association with integrin $\alpha 3 \beta 1$, regulates E-cadherin-mediated cell-cell adhesion. ${ }^{116,117}$ Also, CD151, like its partner integrin $\alpha 6 \beta 4$, is a constitutive component of hemidesmosomes, ${ }^{58}$ a major cell-matrix adhesion machinery in epidermis. At the molecular level, CD151 overexpression leads to the PKC-and Cdc42-dependent actin cytoskeletal reorganization, the process critical for both adhesion and migration, ${ }^{85}$ while CD151 silencing results in excessive RhoA activity and stress fibers formation, ${ }^{117}$ suggesting that CD151 plays important roles in the dynamics and activity of the cytoskeletal machinery in epithelium. In endothelial cells, both overexpression and knockout studies indicate that CD151 can upregulate eNOS, Akt, and Rac activities, which are apparently needed for endothelial cell-cell adhesion and angiogenesis. ${ }^{74,89}$ Endothelial CD151 also promotes the collagenolytic activity and TEM association of MT1-MMP, an ECM remodeling enzyme involved in angiogenesis. ${ }^{49}$

\subsubsection{CD151 Deficiency in Human and Mouse}

\subsubsection{CD151 Deficiency-related Disease in Human}

A truncated human CD151, which is caused by a homozygous nonsense mutation by a nucleotide insertion (G383) in exon 5 of $C D 151$, has been identified in three patients. ${ }^{127}$ This mutation is a frame shift mutation which leads to premature stop codon and thus results in the deletion of amino acid residues 141-253 covering the EC2 and C-terminal domain of CD151, ${ }^{127}$ which are known to be necessary for integrin-binding and CD151 trafficking, respectively. ${ }^{44,61}$ Thus, the truncated protein is dysfunctional 
because it cannot associate with integrins. Patients bearing this mutation exhibited hereditary nephrotic syndrome and developed end stage kidney failure, regional skin lesions, neurosensory deafness, and severe defects in erythropoiesis. ${ }^{127}$ The renal defects are characterized by 1) the thickening and splitting of tubular basement membrane, 2) the thickening, reticulation, and fragmentation of glomerular basement membrane, and 3) the clustering of foam cells in the interstitium. ${ }^{127}$ These findings suggest CD151 is an important regulator in the organization of basement membrane in human kidney.

\subsubsection{CD151 Knockout Mouse}

To date, several research groups have generated and analyzed CD151 knockout mice. The published data show that $C D 151$-null mice are viable and fertile with normal Mendelian inheritance. ${ }^{130}$ The mice show essentially normal tissue morphology including the dermal hemidesmosome organization and skin integrity, unaltered hearing ability, normal blood and bone marrow cell counts, unaffected expression pattern of $\alpha 3$ and $\alpha 6$ integrins in kidney and unchanged expression levels of cell surface $\alpha 3, \alpha 6, \beta 1$, and $\beta 4$ integrin subunits in isolated epidermal keratinocytes. ${ }^{130,136}$ However, CD151 deletion causes some mild abnormalities in 1) hematopoietic cell proportion including a slight increase in the cell numbers of bone marrow promyelocytes and metamyelocytes and a decrease in lymphocyte number, 2) hemostasis including elevation in average bleeding time, average blood loss after injury, and incidence of rebleeding occurrences, and 3) proliferation of $\mathrm{T}$ lymphocytes. ${ }^{130}$

CD151 is expressed by basal keratinocytes in normal skin, and its expression is upregulated at the tip of the migrating epidermis after wounding, suggesting a role in wound healing. ${ }^{67,128}$ Indeed, loss of CD151 results in significantly deficient epidermal wound healing in mice characterized by impaired re-epithelialization. ${ }^{128}$ This defect is very likely due to the aberrant keratinocyte function including proliferation, adhesion, spreading and migration, and/or disorganization of newly formed basement membrane, caused by the loss of CD151. ${ }^{128,137}$

Other observations include the finding that $C D 151$ deletion leads to a strain- and age-dependent glomerular disease in mouse kidney in FVB strain. ${ }^{130,136} \mathrm{CD} 151$ is highly expressed in renal glomeruli and co-localizes with $\alpha 3$ integrin at the base of podocyte foot processes adjacent to the glomerular basement membrane (GBM). ${ }^{136,138}$ In contrast to mice lacking $\alpha 3$ integrin who have severe kidney abnormalities leading to birth lethality, CD151-null mice are neonatally viable and exhibit normal in glomerulus development. ${ }^{136,139}$ However, after several weeks or months, the $C D 151$-null mice displayed mild to severe renal dysfunction, as evidenced by massive glomerular leakage and GBM thickening accompanied by focal glomerulosclerosis, interstitial fibrosis and 
inflammation, and progressive podocyte loss. These pathological changes partially resembles the hereditary nephrotic syndrome in patients who bearing the function-deficient CD151 mutant. ${ }^{127,136,138}$ The abnormalities might result from weakened cell adhesion strengthening of podocytes and deficient GBM assembly and maturation, implicating the roles of CD151 in both podocyte-GBM interaction and GBM organization. ${ }^{136,138}$

With respect to vascular system, the absence of CD151 causes the defects in pathologic angiogenesis in the in vivo Matrigel plug, corneal micropocket, and tumor implantation assays and in the ex vivo aortic ring assay but not in physiologic angiogenesis during development, indicating that CD151 plays an important role in pathologic angiogenesis such as tumor-related angiogenesis and thus contributes to tumor metastasis. ${ }^{74}$ Mechanistically, CD151 promotes pathological angiogenesis by, possibly, regulating the adhesion- and migration-related signaling events including $\mathrm{PKB} / \mathrm{c}-\mathrm{Akt}$, e-NOS, Rac and Cdc42 activities in endothelial cells. ${ }^{74}$

CD151 regulates a variety of cellular and molecular functions based on in vitro studies, but the ablation of CD151 in mouse does not result in similar or comparable phenotypic changes in vivo. This can be explained by the complexity of the in vivo environment or existence of possible compensatory mechanisms for CD151 in vivo. Future studies using genetically modified animals with function-deficient CD151 mutants, such as the dominant negative mutants, might be necessary to better evaluate the roles of CD151 in vivo. On the other hand, compared to $\alpha 3$ integrin deletion-derived deficiencies in wound healing and skin and kidney development, which involves integrin $\alpha 3 \beta 1$-dependent assembly of basement membrane, cell adhesive and migratory abilities, those occurring with CD151-null mice are less severe but still consistent, suggesting the functional relevance and similarity between these two firmly associated proteins in vivo. ${ }^{125-128,136,140}$

\subsection{Summary and Hypotheses}

CD151 associates with laminin-binding integrins and functions as a "facilitator" in integrin-dependent processes and in organization of TEM. It is relatively well established that CD151 promotes tumor metastasis via its regulatory activities toward tumor cell motility, cell adhesion, and MMPs. In vascular endothelial cells, the expression of CD151 is particularly high and throughout vasculature of all human tissues. ${ }^{67}$ A regulatory role of CD151 in pathological angiogenesis had also been determined, ${ }^{74}$ but the precise mechanisms remain unknown. Previous studies have shown CD151 regulates E-cahherin-mediated cell-cell adhesion ${ }^{116,117}$ and integrin-dependent cell-matrix adhesion. ${ }^{58,79,81-83}$ 
We assume that CD151 is involved in regulations of both VE-cadherin-mediated adherens junctions and integrin-mediated cell-matrix adhesion. We also predict that CD151 regulates RhoA-mediated cytoskeletal tension. We therefore hypothesize that CD151 functions as a regulator in coordinating these essential cellular processes and making endothelial cell adhesion and cytoskeletal tension in balance during angiogenic vessel formation and stabilization.

In the present study, we will conduct various studies in vitro and in vivo to evaluate the roles of CD151 in endothelial functions and vascular stability and explore the intracellular signal transductions. Conclusions drawn from the study will help reveal the cellular and molecular mechanism by which CD151 regulates angiogenesis and lead to understanding of the general nature of angiogenesis and vascular stability. 


\section{CHAPTER 2. CD151 STABILIZES VASCULAR NETWORK STRUCTURES AND CONFINES VASCULAR PERMEABILITY}

\subsection{Introduction}

CD151 and its primary binding partner integrin $\alpha 3 \beta 1$ are highly expressed and tightly associated in endothelial cells. ${ }^{49,80}$ Integrin $\alpha 3 \beta 1$ has been shown to be involved in many endothelial cell functions and angiogenesis. ${ }^{141-143}$ Given the function relevance between the two proteins, CD151 might be also important in vascular functions. In this chapter, we provide evidence that CD151 is important in stabilizing the vascular network structures in vitro and promoting vascular barrier function in vivo.

Small interfering RNA (siRNA), which specifically silences protein expression, is a powerful and accessible tool for study of protein functions in cultured cells. ${ }^{144}$ Retroviral-based DNA constructs expressing functional small hairpin RNAs (shRNAs) have been developed and have become the most effective approach to obtain efficient, long-term and stable loss-of-function phenotypes by siRNA. ${ }^{145-147}$ A retrovirus-delivered shRNA system has been reported to knockdown CD151 expression in cancer cells in a highly specific manner. ${ }^{79,148}$ Using this system, we generated stable CD151-silenced endothelial cells and investigated the functions of CD151 in vascular morphogenesis. Furthermore, we isolated primary lung endothelial cells from CD151-null mice and used these cells in this study to substantiate our findings.

Matrigel is a commercial available basement membrane preparation from mouse sarcoma. Previous studies have demonstrated that endothelial cells, after being plated on the top of Matrigel, are capable of forming an anastonosing network of cable-like structures, which has been established as an in vitro model system for vascular morphogenesis. ${ }^{1}$ We took advantage of this model in our study and observed the destabilization of vascular structures caused by the loss of CD151.

The blood vessel wall is largely assembled by a endothelial cell layer containing well-controlled adhesive structures that are required for maintaining tissue homeostasis. ${ }^{149}$ Disruption of the endothelial cell barrier by destabilizing the vascular endothelial adhesive structures elevates vascular permeability and cause plasma leakage. ${ }^{149,150}$ To determine the roles of CD151 in vascular permeability in vivo, we performed a modified Miles assay, a systematical vascular permeability assay, ${ }^{149}$ using CD151-ablated mice. We used mustard oil to induce skin irritation and blood vessel dilation and allow vascular leakage from the skin, and used Evans blue dye to determine the extent of loss-of-CD151-induced vascular permeability. 


\subsection{Materials and Methods}

\subsubsection{Materials}

Matrigel, rat monoclonal anti-mouse PECAM-1 and anti-mouse ICAM-2 antibodies were purchased from BD Biosciences (San Jose, CA). Phalloidin and goat anti-mouse IgG and anti-rabbit IgG secondary antibodies conjugated to Alex Fluor 488 or Alex Fluor 594, and sheep anti-rat IgG conjugated to Dynabeads were obtained from Invitrogen Life Technologies (Carlsbad, CA). Mouse anti-CD151 (5C11) and anti- $\beta 1$ integrin (TS2/16) monoclonal antibodies were described previously. ${ }^{151}$ Puromycin, heparin, mustard oil, Evans blue dye, mineral oil and formamide were obtained from Sigma-Aldrich (St. Louis, MO). Type I collagenase was from Worthington Biochemical Corp. (Lakewood, NJ). Dil-Ac-LDL and endothelial cell mitogen was from Biomedical Technologies Inc. (Stoughton, MD). All other chemicals and reagents were obtained from Sigma-Aldrich unless otherwise indicated.

\subsubsection{Cell Culture and CD151-null Mice}

The retroviral packaging GP2-293 cells were cultured in DMEM (Gibco, Carlsbad, CA) supplemented with 10\% fetal bovine serum (Gibco, Carlsbad, CA), 100 units $/ \mathrm{ml}$ Penicillin and $100 \mu \mathrm{g} / \mathrm{ml}$ Streptomycin.

Immortalized human dermal microvascular endothelial cells (HMECs) were purchased from CDC (Atlanta, GA) and maintained in EBM-2 (Lonza Inc., Allendale, AJ) supplemented with $10 \%$ fetal bovine serum, 100 units $/ \mathrm{ml}$ Penicillin, $100 \mu \mathrm{g} / \mathrm{ml}$ Streptomycin, $1 \mu \mathrm{g} / \mathrm{ml}$ hydrocortisone and $10 \mathrm{ng} / \mathrm{ml}$ EGF (R\&D Systems, Minneapolis, $\mathrm{MN}$ ). Human umbilical vein endothelial cells (HUVECs) were obtained from Lonza Inc. and maintained in EGM supplemented with 10\% fetal bovine serum, 100 units $/ \mathrm{ml}$ Penicillin, $100 \mu \mathrm{g} / \mathrm{ml}$ Streptomycin, 10 units $/ \mathrm{ml} \mathrm{Heparin} \mathrm{and} 3 \mu \mathrm{g} / \mathrm{ml}$ bovine brain extract (Lonza Inc., Allendale, AJ). The endothelial cells were used under passage 10.

The deletion of CD151 in mice was published previously, ${ }^{136}$ and CD151 ${ }^{+/-}$mice (FVB strain) were obtained from A. Sonnenberg lab (the Netherlands Cancer Institute, Netherland). The founder mice were backcrossed more than 5 generations into the C57BL/6J mouse strain, and genotype analysis was performed by PCR as described previously. ${ }^{136}$ A common forward primer has the sequence "GTCTGACCACCCTATTCATTGTC" (primer 1). The reverse primers are "GCATGCTGCCTCACTGAAAGC" (primer 2), which was used to detect wild type alleles, and "GGATGCCAGCAATGCTCTCCA" (primer 3), which was used to detect 
the CD151-deleted alleles. The PCR program was: denaturation at $92^{\circ} \mathrm{C}$ for $2 \mathrm{~min}$ followed by 35 cycles of $1 \mathrm{~min}$ at $92^{\circ} \mathrm{C}, 30 \mathrm{sec}$ at $55^{\circ} \mathrm{C}$ and $30 \mathrm{sec}$ at $72^{\circ} \mathrm{C}$ and a final extension period of $10 \mathrm{~min}$ at $72^{\circ} \mathrm{C} .7$ to 12 week-old $C D 151$-null mice were used for all in vivo and ex vivo assays, and were compared with their wild type littermates of same age and sex.

Mouse lung endothelial cells (MLECs) were isolated as described. ${ }^{74,152}$ Briefly, 8 week-old mice were euthanized, and mouse lungs were excised, rinsed and minced finely with scissors. The lung pieces from 2 mice were then dissociated in $50 \mathrm{ml}$ of $1 \mathrm{mg} / \mathrm{ml}$ collagenase (Type I) with gentle agitation for 1 hour at $37^{\circ} \mathrm{C}$. The tissue suspension was triturated using a $20 \mathrm{cc}$ syringe and then pipetted through a $70-\mu \mathrm{m}$ cell strainer to remove the un-dissociated tissue clumps. Primary endothelial cells were isolated from the pulmonary cell suspension using anti-mouse PECAM-1-coated magnetic Dynabeads. After a 5-day culture period, the cells underwent another selection using anti-mouse ICAM-2-coated Dynabeads to enrich the endothelial cell population. The isolated cells were cultured in MDCB131 medium (Gibco, Carlsbad, CA) supplemented with 10\% fetal bovine serum, 100 units $/ \mathrm{ml}$ Penicillin, $100 \mu \mathrm{g} / \mathrm{ml}$ Streptomycin, $5 \mu \mathrm{g} / \mathrm{ml}$ endothelial cell mitogen, $10 \mathrm{U} / \mathrm{ml}$ heparin, and were used between passages 1 and 3.

The endothelial cell properties from the above preparation were confirmed by the incorporation of Dil-Ac-LDL (acetylated low density lipoprotein labeled with 1,1'-dioctadecyl-3,3,3',3'-tetramethyl-indocarbocyanine perchlorate). ${ }^{153}$ In brief, the isolated primary cells were incubated with complete growth media containing $10 \mu \mathrm{g} / \mathrm{ml}$ Dil-Ac-LDL for 4 hours at $37^{\circ} \mathrm{C}$. The media were then removed, and cells were washed with probe-free media. Then incorporation of the fluorescent probe in vascular endothelial cells was detected by flow cytometry after cell trypsinization.

\subsubsection{Retroviral Production}

A retrovirus-delivered shRNA system, which has been developed to knockdown human CD151 expression in a highly specific manner ${ }^{79,148}$, was used in this study to establish stably CD151-silenced endothelial cells. As shown in Figure 2-1, the CD151-silencing (CD151 KD) pSIREN construct contains a shRNA-expressing cassette targeting the sequence "AGTACCTGCTGTTTACCTACA" in exon 2 of human CD151 transcript, and the non-silencing (MOCK) construct contains a sequence "GCGAGACCATGCCTCCAACAT" which is homologous to sequence in the exon 6 of human CD151 mRNA but does not have gene silencing effect. ${ }^{79,148}$ To produce CD151 $\mathrm{KD}$ and MOCK shRNA construct-containing retroviruses, equal amount of pSIREN construct and $\mathrm{pVSV}-\mathrm{G}$ retroviral coat protein expression vector were co-transfected with into GP2-293 retroviral packaging cells using transfection reagent Lipofectamine 2000 


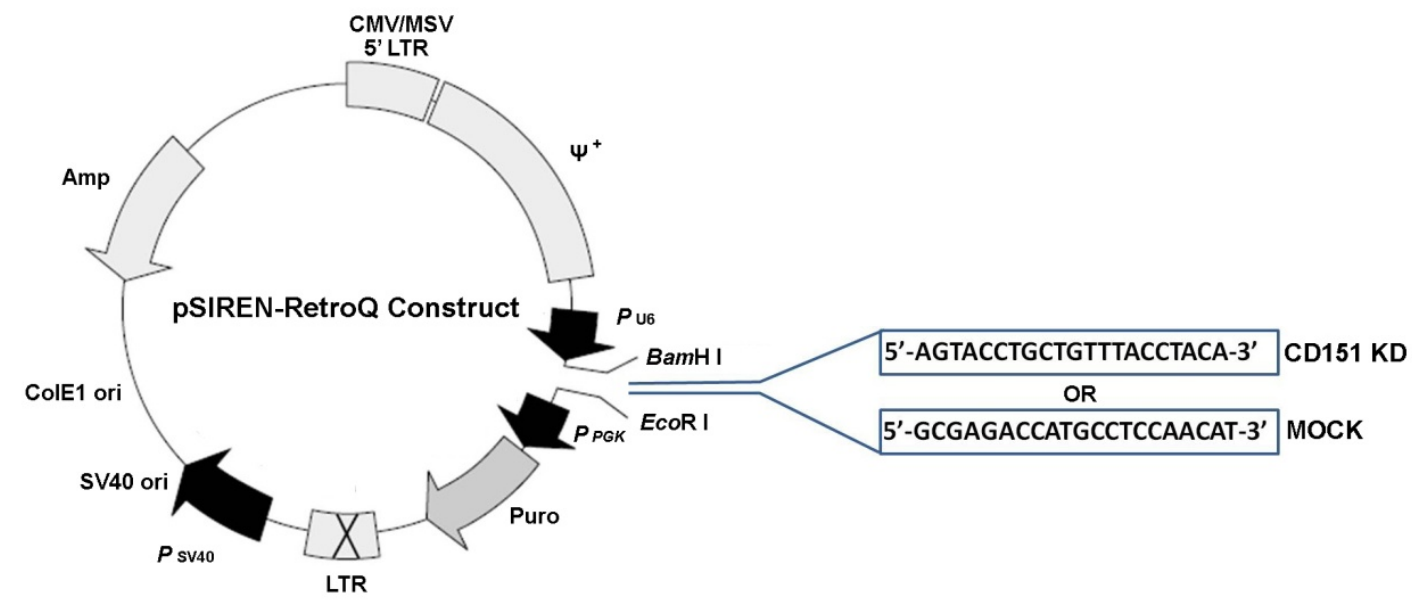

\section{Figure 2-1. ShRNA construct map.}

The CD151 KD or MOCK shRNA oligonucleotide was synthesized and subcloned into the pSIREN RetroQ vector. ${ }^{79,148}$ The CD151-silencing (CD151 KD) shRNA sequence targets the sequence "AGTACCTGCTGTTTACCTACA" in exon 2 of human CD151 transcript. The non-silencing (MOCK) shRNA sequence "GCGAGACCATGCCTCCAACAT" is homologous to sequence in the exon 6 of human CD151 mRNA but does not have gene silencing effect. ${ }^{79,148}$ 
(Invitrogen Life Technologies, Carlsbad, CA). After 4 hours, cells were replenished with fresh DMEM complete medium and cultured for 48 hours. Virus-containing medium was then $0.45-\mu \mathrm{m}$ filtered and stored at $-80^{\circ} \mathrm{C}$ for further experiment.

\subsubsection{Retroviral Transduction and RNAi}

HMECs or HUVECs were seeded at $70 \%$ confluence one day prior to virus exposure. Then virus-containing medium and polybrene (final concentration $5 \mu \mathrm{g} / \mathrm{ml}$ ) were mixed and added to the cell culture dishes. The cells were incubated at $4^{\circ} \mathrm{C}$ for 30 min, then switched back to $37^{\circ} \mathrm{C}$ and incubated for 24 hours. On the second day, cells were feed by fresh medium for a further 24 hours and underwent selection with $2 \mu \mathrm{g} / \mathrm{ml}$ puromycin for puromycin-resistant cells. Stable transductants were obtained after selection, and then subjected to cell sorting to get rid of any RNAi-escaping cell population using anti-CD151 $\mathrm{mAb}(5 \mathrm{C} 11)$ as probe. The sorted cells were maintained with $0.2 \mu \mathrm{g} / \mathrm{ml}$ puromycin. Cells transduced with the non-silencing shRNA-containing viruses served as MOCK cells.

\subsubsection{Flow Cytometry and Immunofluorescence}

For flow cytometry, suspended cells were incubated with $1 \mu \mathrm{g} / \mathrm{ml}$ of various primary Abs including anti-human CD151 (5C11), CD9 (C9BB), CD81 (M38), and integrins $\alpha 3$ (A3-IVA5), $\alpha 5$ (A5-PUJ2), $\alpha 6$ (A6BB), $\alpha \mathrm{V}$ (P3G8) and $\beta 1$ (TS2/16) for 1 hour on ice followed by 3 washes with PBS. Then cells were incubated with appropriate FITC-conjugated secondary antibody for $30 \mathrm{~min}$ followed by 3 more washes. The absence of CD151 on cell surface, as well as expression of other cell surface proteins, was analyzed by a flow cytometer (BD Biosciences).

Immunofluorescence was performed as described with modifications. ${ }^{44}$ In brief, cells were fixed with 3\% paraformaldehyde for $15 \mathrm{~min}$, permeablized with $0.1 \%$ Brij98

for $2 \mathrm{~min}$ and blocked for $30 \mathrm{~min}$, and then incubated with $5 \mathrm{C} 11$ at $4^{\circ} \mathrm{C}$ overnight. Then cells were incubated with Alexa Fluor 594-conjugated secondary antibody and Alexa Fluor 488-conjugated phalloidin for 1 hour. Staining of CD151 and actin in CD151-silenced cells and MOCK cells were visualized using a fluorescent microscope.

\subsubsection{D Capillary Formation Assay}

Matrigel was thawed and maintained as a liquid on ice. Appropriate amount of Matrigel was added to 48-well plates, in duplicate, for regular photography, or in glass 
bottomed cell culture dishes $(1.0 \mathrm{~cm}$ in diameter of the glass bottom cell culture area) for time-lapse video microscopy, and incubated at $37^{\circ} \mathrm{C}$ for 1 hour. HMEC transfectants or the mouse lung endothelial cells were seeded on the top of Matrigel in a 60,000/well density. The cellular cables were photographed at different time points or recorded by time-lapse video microscopy every 5 min for 72 hours. The total cable length and/or the number of cable cord-enclosed regions within the photographs and videos were calculated and presented as quantitative data for the cable network structures.

\subsubsection{Modified Miles Assay}

The modified Miles assay, which is used to determine vascular permeability in vivo, was described previously. ${ }^{149}$ Evans blue dye $(30 \mathrm{mg} / \mathrm{kg}$ in $100 \mu \mathrm{lPBS})$ was injected i.v. into 12 week-old male WT and CD151 KO mice through the retro orbital sinus using a 30-gauge needle. The 5\% mustard oil in mineral oil (v/v) or mineral oil alone was immediately administrated on the dorsal and ventral surfaces of the ear. The administration was repeated one more time 15 min later. After Evans blue had circulated for $30 \mathrm{~min}$, the mice were anesthetized and perfused with $10 \mathrm{ml}$ PBS. Mouse ear was then photographed, excised, dried and weighed. Evans blue dye was extracted in $1 \mathrm{ml}$ formamide overnight at $55^{\circ} \mathrm{C}$ and its content in the ears was quantified by measuring the absorbance at $610 \mathrm{~nm}$ with a spectrophotometer.

\subsection{Results}

\subsubsection{Silencing CD151 Expression in Endothelial Cells}

A retrovirus-delivered shRNA system has been developed to knockdown human CD151 expression in a highly specific manner. ${ }^{79,148}$ We have established 1) the stable transductant of CD151 knockdown (CD151 KD) and 2) the control transductant (MOCK) in HMECs. Compared with the parental HMECs and MOCK cells, the CD151 KD cells displayed 1) a near total loss of CD151 expression at the cell surface in flow cytometry (Figure 2-2A) and 2) remarkable downregulation of CD151 expression in immnofluorescence assays (Figure 2-2B). In addition, HUVEC cells with efficient CD151-silencing were also successfully obtained by the shRNA retroviral transduction (data not shown). 
A
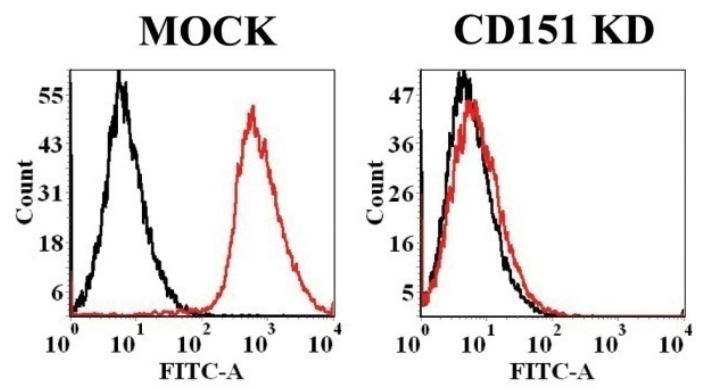

- Mouse IgG

- CD151

B

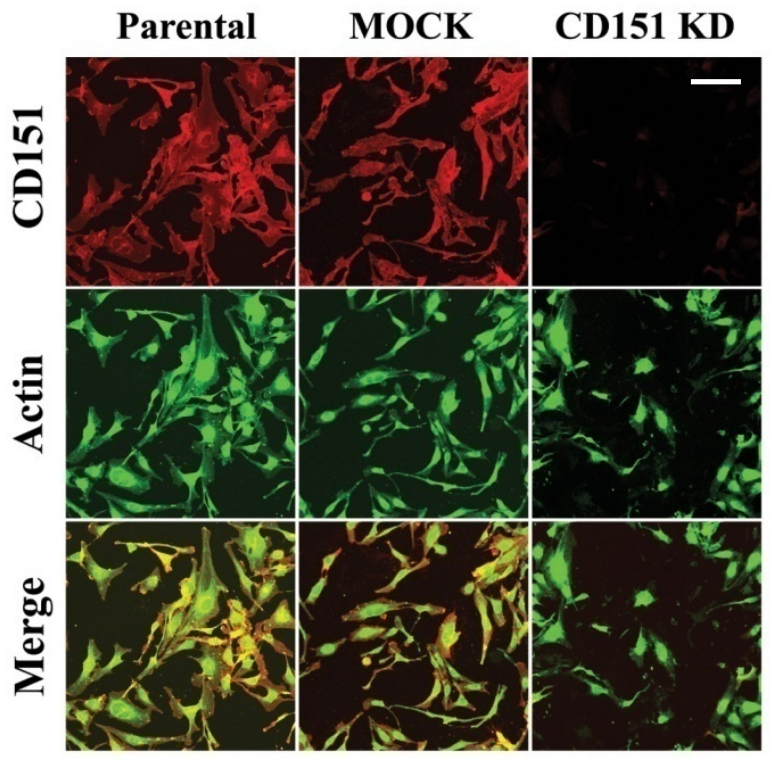

Figure 2-2. Silencing of CD151 in HMECs.

HMEC cells were transduced with CD151 knockdown (KD) and MOCK shRNA-encoding retroviruses, followed by selection with $2 \mu \mathrm{g} / \mathrm{ml}$ puromycin. CD151 $\mathrm{KD}$ transductants were then subjected to cell sorting to get rid of any RNAi-escaping cells, and the silencing efficiency was determined by (A) flow cytometry and (B) immunofluroscence staining using CD151 mAb (5C11). Bar $=50 \mu \mathrm{m}$. 


\subsubsection{CD151 Silencing Does Not Alter Cell Surface Expression of CD151-related Integrins and Tetraspanins}

CD151 physically and/or functionally associates with integrins and other tetraspanins to form TEMs. ${ }^{31}$ Hence, the expressions of these transmembrane proteins are important for CD151 functions in endothelial cells. We analyzed the cell surface expressions of various integrins and tetraspanins related to CD151 using flow cytometry and found no significant differences in the expressions of integrins $\alpha 3, \alpha 5, \alpha 6, \alpha \mathrm{V}$, and $\beta 1$ and tetraspanins CD9 and CD81 in HMEC-parental, -MOCK, and -CD151 KD cells (Table 2-1). These results indicate the expressions of these integrins and tetraspanins in HMEC and suggest that neither retroviral transductions nor CD151 knockdown alters the cell surface expressions of these CD151-relevant proteins. The results also confirm that the CD151-silencing system is highly specific.

\subsubsection{Identification of Isolated MLECs}

Dil-Ac-LDL is a vascular endothelial cell and macrophage-specific fluorescent dye. After uptake of Dil-Ac-LDL, the lipoprotein is degraded within lysosome and the

intracellular membranes of the cells are labeled with Dil (a PE-like fluorescent probe). ${ }^{153}$ Macrophages can be differentiated from endothelial cells because they are more brightly labeled. Thus, we used Dil-Ac-LDL to identify the primary endothelial cells we prepared from mouse lung using anti-mouse PECAM-1 and ICAM-2, the two endothelial cell-specific markers, monoclonal antibodies. As shown in Figure 2-3, most of the cells (more than 90\%) isolated from both WT and CD151 KO mouse lung are single peaked and labeled with Dil at relatively low level, indicating that 1) no contamination of macrophages, and 2) the high purity of vascular endothelial cells in the isolated cells. These results show that we have obtained mouse WT and CD151 KO primary endothelial cells with high purity.

\subsubsection{CD151 Stabilizes Vascular Network Structures Formed by Endothelial Cells on Matrigel}

In this study, we used the model system "3D capillary formation on Matrigel" to evaluate the roles of CD151 in vascular morphogenesis. As shown in Figure 2-4, both HMECs and MLECs initially formed similar capillary-like network structures on Matrigel regardless of the presence and absence of CD151 expression. Neither HMECs nor MLECs show significant difference in total cable length between CD151 expressing (HMEC-MOCK and MLEC-WT) and non-expressing (HMEC-CD151 KD and MLEC-CD151 KO) endothelial cells, suggesting the initial formation of capillary 
Table 2-1. The surface expression of integrins and tetraspanins on HMEC-parental, -MOCK, and -CD151 KD cells.

\begin{tabular}{llll}
\hline Transductants & Parental & MOCK & CD151 KD \\
\hline Mouse IgG & $220 \pm 39$ & $226 \pm 37$ & $218 \pm 53$ \\
CD151 & $3,098 \pm 113$ & $3,244 \pm 221$ & $290 \pm 56$ \\
CD9 & $10,156 \pm 1,132$ & $11,018 \pm 764$ & $10,245 \pm 1,609$ \\
CD81 & $6,644 \pm 414$ & $7,297 \pm 760$ & $7,814 \pm 833$ \\
Integrin $\alpha 3$ & $5,431 \pm 445$ & $5,618 \pm 655$ & $4,801 \pm 221$ \\
Integrin $\alpha 5$ & $1,018 \pm 81$ & $1,110 \pm 96$ & $1,012 \pm 197$ \\
Integrin $\alpha 6$ & $1,452 \pm 291$ & $1,479 \pm 346$ & $1,529 \pm 207$ \\
Integrin $\alpha \mathrm{V}$ & $1,594 \pm 583$ & $1,990 \pm 960$ & $1,447 \pm 428$ \\
Integrin $\beta 1$ & $16,747 \pm 763$ & $17,878 \pm 662$ & $18,072 \pm 531$ \\
\hline
\end{tabular}

Note: The flow cytometry analysis of HMECs was performed using mAbs against human tetraspanin CD151 (5C11), CD9 (C9BB), or CD81 (M38) and integrin $\alpha 3$ (A3-IVA5), $\alpha 5$ (A5-PUJ2), $\alpha 6$ (A6BB), $\alpha \mathrm{V}$ (P3G8), or $\beta 1$ (TS2/16) to confirm the absence of CD151 on cell surface as well as expression of other cell surface proteins. The numbers represent the mean fluorescence intensities \pm standard deviation from 3 independent experiments. 


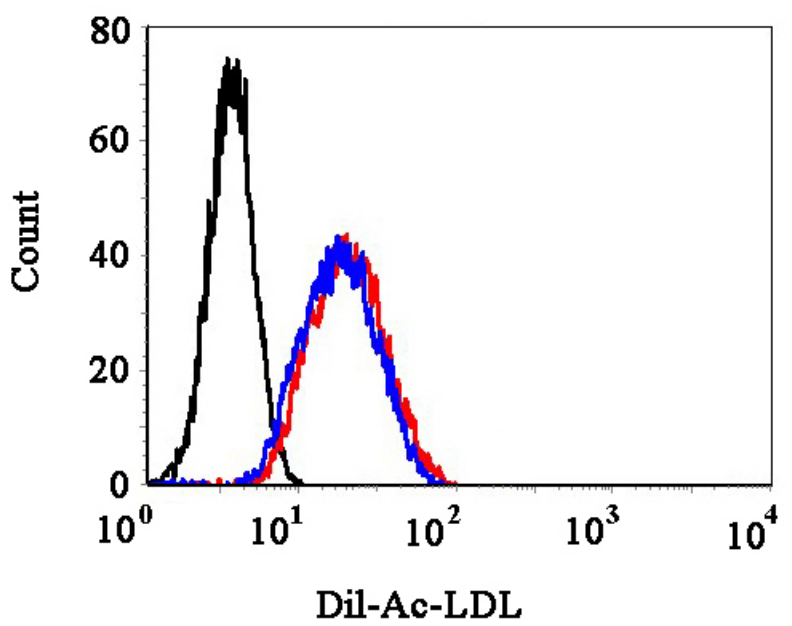

\section{- Negative \\ - MLEC-WT \\ - MLEC-CD151 KO}

\section{Figure 2-3. Identification of MLECs.}

The isolated lung primary endothelial cells were incubated with complete growth media containing $10 \mu \mathrm{g} / \mathrm{ml}$ Dil-Ac-LDL for 4 hours at $37^{\circ} \mathrm{C}$. Cells were trypsinized and washed, and the incorporation of the fluorescent dye by cells was analyzed by flow cytometry. 
A

HMEC
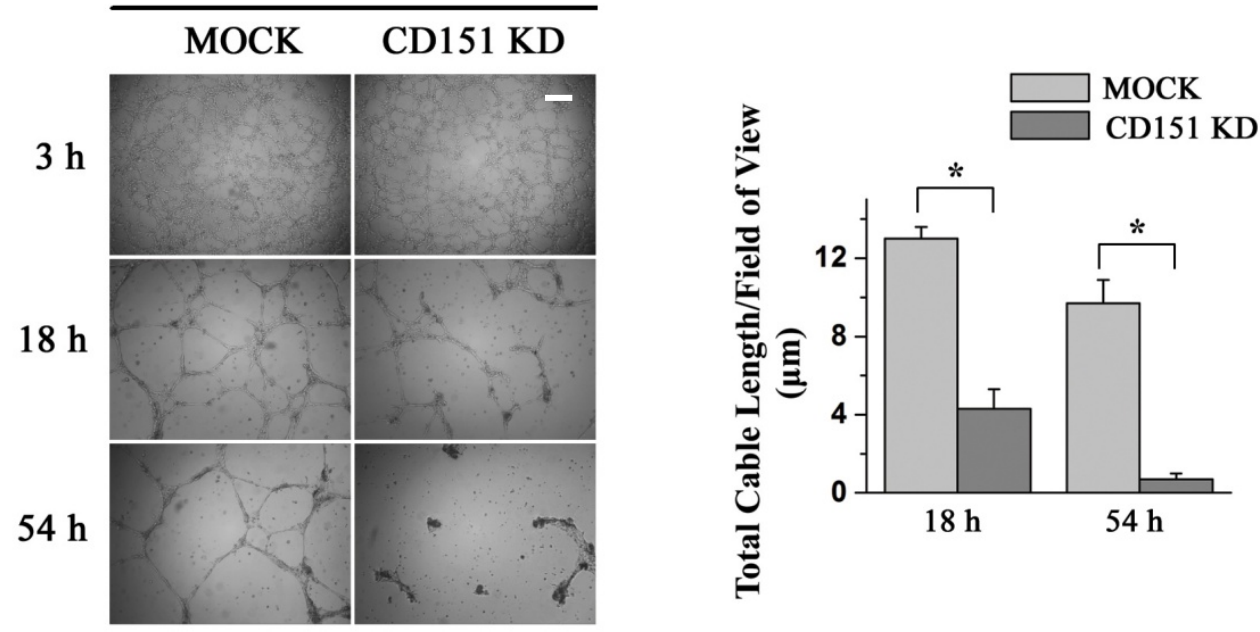

B

MLEC
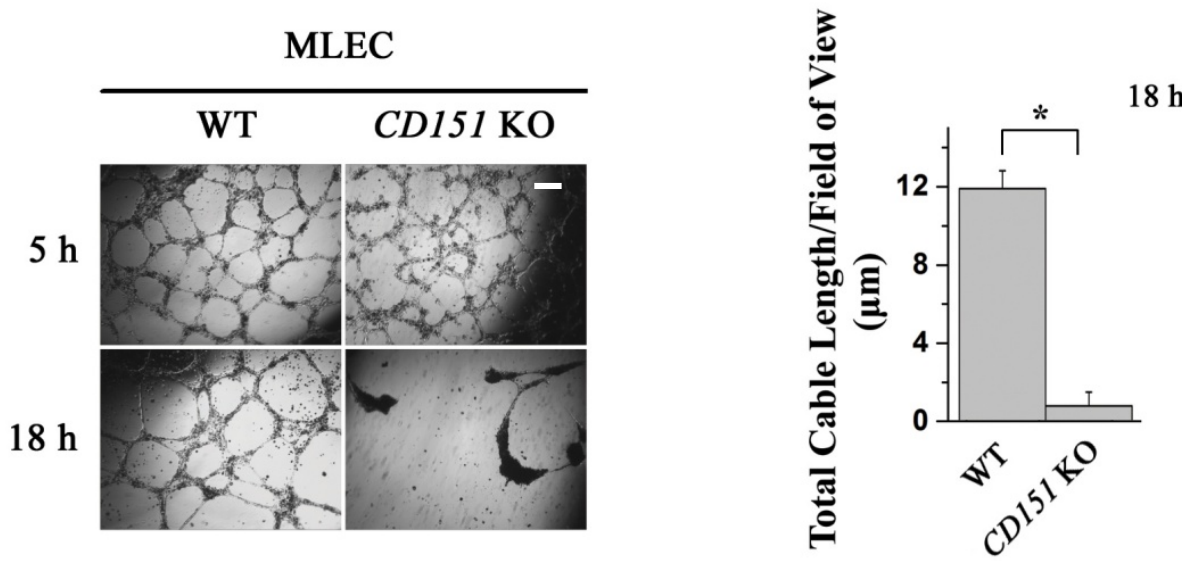

Figure 2-4. Loss of CD151 impairs vascular stability.

(A) Silencing of CD151 expression disrupted the capillary-like structures on Matrigel. HMEC-Mock and -CD151 KD cells were plated on Matrigel, and photographed at the indicated time points. (B) Deletion of CD151 in mice impairs angiogenesis in vitro. Mice lung endothelial cells (MLEC) were isolated from wilt (WT) and CD151 null (CD151 $\mathrm{KO})$ mice and employed in the Matrigel cable formation assay. Representative photographs are shown for HMECs and MLECs. Bar $=250 \mu \mathrm{m} . * \mathrm{P}<0.01$. 
network is not affected by the loss of CD151. However, as time goes by, CD151-silenced and CD151-null cells cannot maintain the cable-like structures. Compared with the network structure formed by CD151-expressing cells (HMEC-MOCK and MLEC-WT), the ones formed by CD151-silenced (HMEC-CD151 KD) and CD151-null (MLEC-CD151 KO) cells were markedly and largely disrupted at 18 hour, respectively. In CD151-silenced HMECs, the networks became completely lost at 54 hour. The quantitative results for the cable-like structures reveal the disruption caused by the loss of CD151, as indicated by the remarkable decrease in total cable length of the cable network in HMEC-CD151 KD (Figure 2-4A) and MLEC-CD151 KO cells (Figure 2-4B).

Using DIC time-lapse video-microscopy, we found that HMEC-MOCK and -CD151 KD cells were attached and spread equally well and actively migrated around soon after being plated on Matrigel (Supplement Movies). In the following 2-4 hours, both MOCK and CD151 KD cells assembled into networks of cable structures to the similar extent, suggesting that CD151 is not required for endothelial cells patterning into vascular structures. In general, MOCK cells can maintain the network structures for several days though the cables become thicker and denser. In contrast, CD151 KD cells cannot maintain the network structure, and the cable networks were continually contracting and eventually broke into disconnected cell clumps. The quantitative results of the capillary structures from the videos, presented as the number of circled regions formed by the cable cords at different time point (Figure 2-5), show significant difference between MOCK and CD151 KD cells. MOCK cells formed much more cable cord-enclosed areas at $\sim 5$ hour and afterwards than CD151 KD cells, indicating MOCK cells form more stable network structures than CD151 KD cells, and suggesting loss of CD151 unstabilizes the vascular network structures formed by endothelial cells.

Together, these results demonstrate that CD151 stabilizes the newly formed endothelial network structures without affecting the initial formation of the vascular structures.

\subsubsection{CD151 Regulates Vascular Permeability}

To substantiate the in vitro finding, we then analyzed the vascular stability of CD151 knockout mice, ${ }^{136}$ by performing a modified Miles assay, which examines microvascular permeability during inflammation. ${ }^{149}$ As shown in Figure 2-6, compared with mineral oil alone group, the 5\% mustard oil-treated group shows substantial vascular permeability on mouse ear after 30-min circulation of Evans blue dye, as indicated by dermal leakage of the blue dye in the ear. On the other hand, the level of dermal dye leakage is significantly higher in CD151 KO mice than that in WT mice, based on the quantitative data from 30 mice. This data suggests that vascular permeability is 


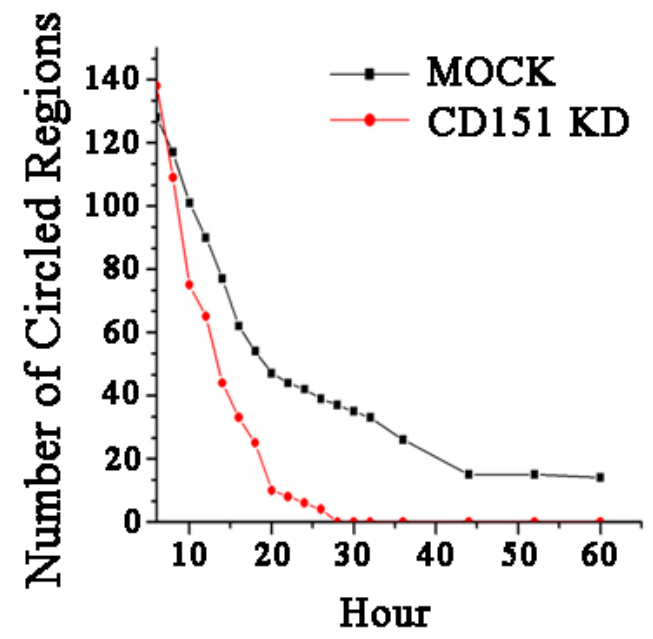

Figure 2-5. Quantification of the cable network in the time-lapse movies.

The number of cable cord-enclosed regions in the movies was counted at different time points.
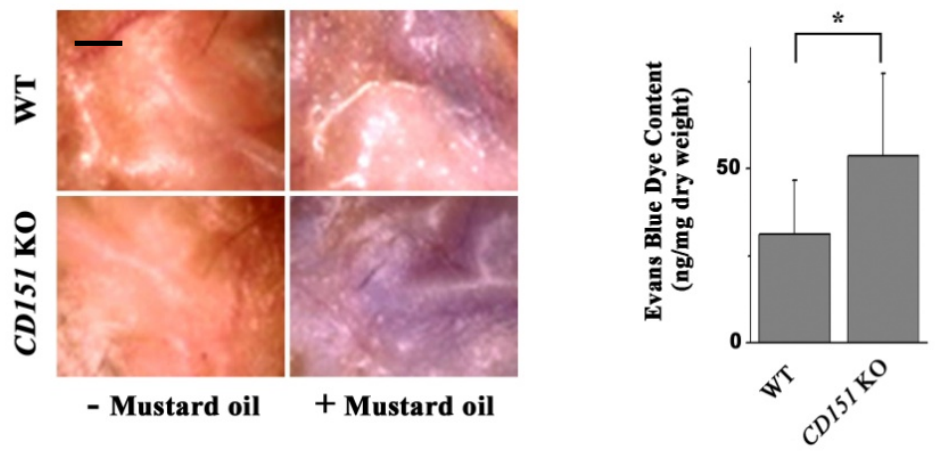

Figure 2-6. Loss of CD151 increases vascular permeability in mice.

Evans blue dye ( $30 \mathrm{mg} / \mathrm{kg}$ in PBS) were injected in 12 week-old male mice i.v.. Mustard oil in mineral oil $(5 \% \mathrm{v} / \mathrm{v})$ or mustard oil alone was applied to the dorsal and ventral surfaces of the ears twice, at 0 min and 15 min later. After 30 min circulation, the mice were perfused with PBS. Then dye leakage area at the ventral surface of the ear was photographed, and the content of dye in ears was determined after extraction. Bar $=1 \mathrm{~mm}$. $* \mathrm{p}<0.05$. 
markedly elevated in $C D 151 \mathrm{KO}$ mice, compared with wild type littermates upon the inflammatory factor mustard oil treatment. We conclude that CD151 is important in preventing vascular leakage and maintaining vascular integrity and stability.

\subsection{Summary}

In order to investigate the roles of CD151 in vasculature, we established stably CD151-silenced endothelial cell lines using a retrovirus-delivered shRNA. A near total loss of CD151 expression was obtained in these cell lines. We found the CD151 shRNA system functions in a highly specific manner since CD151 silencing does not alter the cell surface expression levels of its related integrins and other tetraspanins. In addition, we prepared lung primary endothelial cells from WT and CD151 KO mice for further mechanistic studies.

We took advantage of the in vitro model system, 3D capillary formation on Matrigel, to evaluate the biological functions of CD151 in vascular morphogenesis We have found that loss of CD151 alters vascular stability. In the absence of CD151, endothelial cells can initiate the formation of capillary network structures on Matrigel; however, as time goes by, the vascular structures cannot be sustained. Furthermore, we performed an in vivo microvascular permeability assay, and observed significantly elevated vascular permeability induced by mustard oil in CD151 KO mice. Collectively, our results demonstrated that CD151 stabilizes vasculatures without affecting the initial formation of the vascular network structures. 


\section{CHAPTER 3. CD151 STRENGTHENS ENDOTHELIAL CELL-MATRIX ADHESION WITHOUT AFFECTING CELL DEATH AND MOTILITY}

\subsection{Introduction}

Angiogenesis is a complex process involving endothelial cell activation and migration from pre-existing blood vessels, cell proliferation and assembly of endothelial cells into an well-controlled intercellular structures and attachment on basement membrane. ${ }^{2,3,154}$ We have shown loss of CD151 disrupts the maintenance of the capillary-like structures on 3D Matrigel and results in aberrant vascular permeability in vivo. From the results shown in Figure 2-4, the time-lapse videos and other studies demonstrating that CD151 plays important roles in cell migration, cell-cell adhesion, cell morphogenesis, and adhesion strengthening in epithelial or tumor cells, we predict that CD151 might be also important in the basic endothelial cellular processes including cell death, cell motility, cell-matrix adhesion, cell-cell adhesion and cytoskeletal tension, and thus participate in the regulation of vascular stability. In Chapters 3 to 5, we will examine the roles of CD151 in these cellular processes and the associated signaling events and define the precise mechanisms by which CD151 regulates vascular stability.

Since endothelial cell growth and motility play important roles in angiogenesis, ${ }^{2,3,154}$ we first performed a set of assays for cell death and cell motility to evaluate the roles of CD151 in these cell events.

Cell-matrix adhesion is primarily mediated by integrins. After being engaged with ECM, integrins induce formation of focal adhesions and cortical actin cytoskeleton assembly. ${ }^{155,156}$ In focal adhesions, integrins associate with a group of focal adhesion-associated adaptor proteins, such as paxillin and focal adhesion kinase (FAK), and the actin cytoskeletal "crosslinker" proteins including vinculin, $\alpha$-actinin and talin to mediate the anchorage of actin filaments. ${ }^{155-158}$ To elucidate the role of CD151 in endothelial cell-matrix adhesion, we first performed conventional, short-term, static cell-matrix adhesion assay to investigate integrin-mediated early attachment of endothelial cells onto various matrices. Furthermore, we employed traction force microscopy (TFM) to determine the effect of loss of CD151 on cell-matrix adhesion in high resolution. Also as known, within focal adhesions, integrins serve as the transmembrane mechanical linkages between ECM and cytoskeleton by binding to the ECM components with their extracellular domains and to cytoskeletal proteins with the cytoplasmic domain of the $\beta$-subunit. ${ }^{155,156}$ Thus, the soundness of focal adhesions is dependent not only on the ligand binding affinity of integrins but also on their intracellular cytoskeletal connections. Therefore, we also analyzed connections of $\beta 1$ integrin with actin cytoskeleton to obtain in-depth mechanistic information. In addition, 
because CD44 binds to hyaluronan, connects with actin cytoskeleton through ezrin/radixin/moesin (ERM) proteins, a different type of protein "linkers" for the anchorage of cytoskeleton, and mediates cell-matrix adhesion, ${ }^{159-162}$ we also investigated the effect of CD151 on CD44 expression.

\subsection{Materials and Methods}

\subsubsection{Materials}

Propidium iodide (PI) was purchased from Calbiochem (San Diego, CA). Fibronectin and laminin 1 were obtained from Invitrogen Life Technologies (Carlsbad, CA). Laminin 5 was described previously. ${ }^{163}$ Fluorescent latex beads (FluoSpheres 0.2 $\mu \mathrm{m})$ were purchased from BD Biosciences (San Jose, CA). Sulfo-SANPAH was from Thermo Fisher Scientific (Rockford, IL). Mouse anti- $\beta$-tubulin mAb was from Sigma-Aldrich (St. Louis, MO). Mouse anti-CD44 mAb was purchased from BD Biosciences (San Jose, CA). Goat anti-mouse and anti-rabbit IgG antibodies conjugated to horseradish peroxidase (HRP) were obtained from Amersham Biosciences Corp. (Piscataway, NJ). Aprotinin and leupiptin were from Roche Diagnostics (Indianapolis, IN). All other chemicals and reagents were obtained from Sigma-Aldrich unless otherwise indicated.

\subsubsection{Cell Death Assay}

PI staining and flow cytometry were performed to analyze cell death. HMEC transductants were plated in 6-well plate which was precoated with diluted Matrigel. After cultured for 18 hours or 54 hours, cells were trypsinized and washed with PBS 3 times. Then cells were resuspended in PI buffer ( $1 \mu \mathrm{g} / \mathrm{ml}$ in PBS containing $2 \%$ FBS) and incubated on ice for $45 \mathrm{~min}$ in dark. Dead cells were stained brightly red by PI and were analyzed by FACS analysis.

\subsubsection{Cell Motility Assays}

For transwell migration assay, the outer side of the transwell polycarbonate filter (0.8- $\mu \mathrm{m}$ pore, Costar) were precoated with various ECM components $(10 \mu \mathrm{g} / \mathrm{ml}$ fibronectin, $10 \mu \mathrm{g} / \mathrm{ml}$ laminin 1 and $2 \mu \mathrm{g} / \mathrm{ml}$ laminin 5) at $4^{\circ} \mathrm{C}$ overnight and then blocked by $0.1 \%$ heat-inactivated (HI) BSA for 1 hour at $37^{\circ} \mathrm{C} .2,000$ HMEC cells suspended in $300 \mu \mathrm{l}$ serum-free medium containing $0.1 \% \mathrm{HI} \mathrm{BSA}$, in triplicate, were then plated into 
the upper chamber of the transwell filter. $300 \mu \mathrm{l}$ of complete medium were added into the lower chamber as attractive medium. Cells were allowed to migrate at $37^{\circ} \mathrm{C}$ for 4 hours. Then those cells staying in upper chamber were removed with cotton swabs and those cells that migrated to the side towards lower chamber were stained and counted.

Wound healing assay was performed as described. ${ }^{164}$ In brief, HMEC-MOCK or -CD151 KD cells were plated in 24-well plate and cultured until confluence. Then cells were starved for 24 hours before wound formation. Wounded areas were created in monolayer of cells by scraping with a $200-\mu$ l pipette tip. The wounded cell monolayer was cultured in serum-free media and the changes of cell-free area were photographed at different time points under a microscope connected with a digital camera.

\subsubsection{Cell-matrix Adhesion Assay}

Cell-matrix adhesion assay was performed in a 96-well format as described. ${ }^{165}$ Briefly, the wells of 96-well plates were precoated with $10 \mu \mathrm{g} / \mathrm{ml}$ fibronectin, $10 \mu \mathrm{g} / \mathrm{ml}$ laminin $1,2 \mu \mathrm{g} / \mathrm{ml}$ laminin 5 or diluted Matrigel at $4^{\circ} \mathrm{C}$ overnight and then blocked by $0.1 \% \mathrm{HI}$ BSA for 1 hour at $37^{\circ} \mathrm{C} .10,000 \mathrm{HMEC}$ cells suspended in $100 \mu \mathrm{l}$ serum-free media containing $0.1 \% \mathrm{HI}$ BSA were plated into the wells in triplicates. After 35-min incubation at $37^{\circ} \mathrm{C}$, non-adherent cells were removed by 3 gentle washes with PBS. The adhered cells were photographed and counted from each photograph.

\subsubsection{Traction Force Microscopy}

The cellular traction forces were measured by traction force microscopy based on the experimental and computational advances as described previously ${ }^{166,167}$ with modifications.

Briefly, HMECs were placed on the fluorescent latex beads-embedded polyacrylamide (PAA) gel, which were coated with sulfo-SANPAH to conjugate fibronectin $(10 \mu \mathrm{g} / \mathrm{ml}$ in PBS) or laminin $1(10 \mu \mathrm{g} / \mathrm{ml}$ in PBS $)$, and allowed to attach and spread for 12-24 hour prior to image. Individual cells were imaged with both phase contrast and confocal microscopy to capture the positions of the fluorescent beads under and around cell area while the cell exerts traction forces on PAA gel upon attaching. The cells were then detached by trypsinization and a second confocal image of the bead-embedded substrate was taken to capture the positions of the fluorescent beads in the absence of cellular traction forces. Then the displacement field caused by cellular traction forces was obtained from the High Density Mapping software analysis ${ }^{166}$ of the two images using the phase correlation method, which is based on the Fourier shift 
principle. Finally, the displacement data was calculated into the traction field using the

Fourier-transform traction cytometry (FTTC) method as described. ${ }^{167,168}$

The traction force contour plots were generated from the traction field data for each cell area. The maximum traction force within each cell area was calculated as the maximum traction force magnitude value averaged by the surrounding vector magnitudes after a peak smoothing algorithm processing. The average perimeter stress was calculated as the average of the reaction force values found along the perimeter of the cell. For each transfectant, 10-15 cells were measured, and comparisons were made for both maximum and average traction stress magnitudes using a non-parametric Mann-Whitney test, with $\mathrm{p}<0.05$ indicating significant difference.

\subsubsection{TIRF Microscopy}

HMEC transductants were plated on glass bottom dishes and cultured overnight. Cells were then fixed with freshly prepared 3\% paraformaldehyde for 15 min without permeabilization, blocked with $20 \%$ goat serum for $30 \mathrm{~min}$, and incubated with either anti- $\alpha 3$ integrin (A3-IVA5) or anti-CD9 (C9BB) $\mathrm{mAb}$ at $4^{\circ} \mathrm{C}$ overnight. Then cells were incubated with Alexa Fluro 488-conjugated secondary antibody for 1 hour. Staining of $\alpha 3$ integrin and CD9 at basal surface of CD151-silenced cells and MOCK cells were analyzed using a TIRF microscope.

\subsubsection{Detergent Solubility Assay for $\beta 1$ Integrin}

To analyze the connection of $\beta 1$ integrin to actin cytoskeleton, the detergent solubility assay were performed as described previously. ${ }^{85}$ In brief, HMEC transductants were cultured overnight on fibronectin-precoated dishes and then solubilized with $0.15 \%$ Triton X-100 in HEPES buffer on ice for 10 min with gentle pipetting. After centrifugation at 100,000 g for $30 \mathrm{~min}$, the supernatants were mixed with equal volume of 2 X Laemmi buffer and labeled as the soluble fractions. The pellets were dissolved in 1 $\mathrm{X}$ Laemmi buffer and labeled as the detergent-insoluble fractions. Total cellular was extracted by $1 \mathrm{X}$ Laemmi buffer from uncentrifuged raw cell lysates. Integrin $\beta 1$ distributed in soluble and insoluble fractions as well as total cellular $\beta 1$ integrin was resolved by reducing SDS-PAGE. The separated proteins were then transferred to 0.45 $\mu \mathrm{m}$ PVDF membrane and subjected to immunoblotting. For immunoblotting, the membrane were blocked with 5\% non-fat milk at room temperature for $30 \mathrm{~min}$, probed with $\beta 1$ integrin $\mathrm{mAb}$ (TS2/16) at $4^{\circ} \mathrm{C}$ overnight, and developed with HRP-conjugated secondary antibody followed by chemiluminescence. 


\subsubsection{TS2/16 Antibody Treatment}

HMEC transfectants were pre-incubated with $1 \mu \mathrm{g} / \mathrm{ml} \mathrm{TS} 2 / 16$, a $\beta 1$ integrin-activating antibody, in cell culture medium for 1 hour on ice, and then used in 3D capillary formation assay as described in Section 2.2.6. The cells were replenished with fresh medium containing $1 \mu \mathrm{g} / \mathrm{ml} \mathrm{TS} 2 / 16$ every 8 hours and photographed at indicated time points. No antibody- and anti-CD 71 antibody (an isotype-matched $\mathrm{mAb}$ )-treated cells served as negative controls. Total cable length and number of circled regions were counted and used as quantitative data for the cable network structures.

\subsubsection{Analysis of CD44 Expression}

The mRNA expression levels of CD44 in HMEC transfectants were determined by quantitative real time-PCR (qRT-PCR). Total RNA was extracted from HMEC transductants in triplicates using RNase Protect Mini Kit (Qiagen, Valencia, CA), according to the manufacturer's instructions. RNA concentration was determined by measuring the absorbance at $280 \mathrm{~nm}$ using a NanoDrop ND-1000 spectrophotometer. Total mRNA was then immediately reverse-transcribed into cDNA using a Taqman Reverse Transcription Reagent Kit (Applied Biosystems, Carlsbad, CA). RT-PCR was performed on cDNA template with a Roche Light Cycler instrument according to manufacturer's instruction. An intron-spanning $92 \mathrm{nt}$ amplicon of human CD44 gene was obtained from qRT-PCR using the forward primer "gtgttcgtgccagctatgac" and reverse primer "ccatcattgattgtctccacac", which are located at CD44 exons 1 and 2, respectively. The internal control RT-PCR was set to the reference gene, human TATA Boxing Protein (TBP), for the cDNA sample using forward primer "GACACCATGGACAAGTTTTGG" and reverse primer "CGGCAGGTTATATTCAAATCG". The Ct (cycle threshold) values were obtained from every PCR test. ${ }^{169}$ The expression levels of CD44, presented as relative mRNA levels, were calculated according the equation as:

$$
E=2^{-[C t(I)-C t(R)]}
$$

where $\mathrm{E}$ is defined as relative mRNA expression level. ${ }^{169} \mathrm{Ct}(\mathrm{I})$ and $\mathrm{Ct}(\mathrm{R})$ are the $\mathrm{Ct}$ values of gene of interest and of reference gene, respectively. Then data were normalized by setting the relative CD44 mRNA levels in HMEC-MOCK cells as 1, and comparison was performed between MOCK and CD151 KD cells. Cell surface and total cellular levels of CD44 protein was analyzed by flow cytometry and immunoblotting using CD44 $\mathrm{mAb}$, respectively. 


\subsection{Results}

\subsubsection{CD151 Does Not Affect Cell Death}

Decreased stability of CD151-silenced and -ablated endothelial network may result from increased apoptosis and reduced cell proliferation rate. We assessed the effect of CD151 silencing on cell survival. As shown in Figure 3-1, both HMEC-MOCK and -CD151 KD cells exhibited almost full viability after being plated on Matrigel for 18 hours or 50 hours, indicating that the vascular instability in the absence of CD151 is not a result of cell death. Study from elsewhere demonstrated that CD151 removal has no impact on endothelial cell proliferation. ${ }^{74}$

\subsubsection{Endothelial Cell Migration Remains Unchanged in the Absence of CD151}

CD151 promotes cell movement in a variety of cell systems. ${ }^{72,79}$ However, the loss of CD151 expression in HMECs did not markedly affect cell migration. Neither the ability of cell to migrate onto fibronectin, laminin 1, and laminin 5 in transwell migration assay (Figure 3-2A) nor the cell motility in wounding healing assay (Figure 3-2B) was significantly altered in CD151 KD cells, compared to MOCK cells, suggesting that endothelial cell migration is not responsible for CD151-dependent maintenance of endothelial network structures during HMEC morphogenesis.

\subsubsection{CD151 Is Needed for Optimal Endothelial Cell-matrix Adhesiveness}

To fully understand the functions of CD151 in endothelial cell-matrix adhesion, we first evaluated the effect of loss of CD151 on the early adhesion of HMECs on various ECM using the conventional cell-matrix adhesion assay. As shown in Figure 3-3A, more MOCK cells were resistant to the washing shear forces than CD151 KD cells 35 min after being plated on laminin 5, suggesting loss of CD151 diminished cell adhesiveness at the early stage of endothelial cell adhesion onto laminin 5. However, no significant decreases in early adhesion onto fibronectin, laminin 1 and Matrigel were found in CD151 KD cells. This result is consistent with earlier observations in other cells $^{74,79}$ and underlines that CD151 only markedly attenuates the early adhesion dependent on laminin 5-binding integrins $\alpha 3 \beta 1$ and/or $\alpha 6 \beta 4$ to a magnitude detectable via shear force-induced detachment.

The traction forces, that take place between cells and the surrounding environments, are generated by the actomyosin cytoskeleton and transmitted to the 


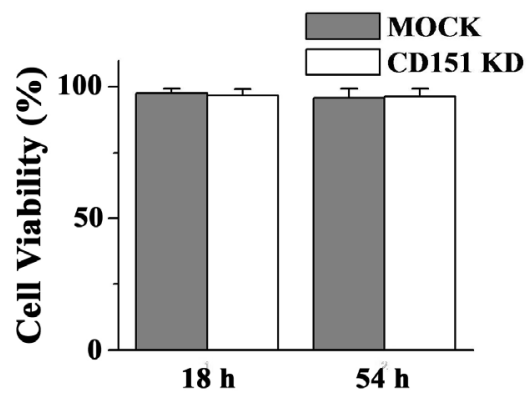

Figure 3-1. Loss of CD151 does not affect endothelial cell viability.

HMEC transductants were plated on diluted Matrigel-precoated 6-well plate and cultured for 18 or 54 hours. Cells were incubated with $1 \mu \mathrm{g} / \mathrm{ml} \mathrm{PI}$, and the stained cells were then analyzed by flow cytometry to analyze dead and live cells. 
A
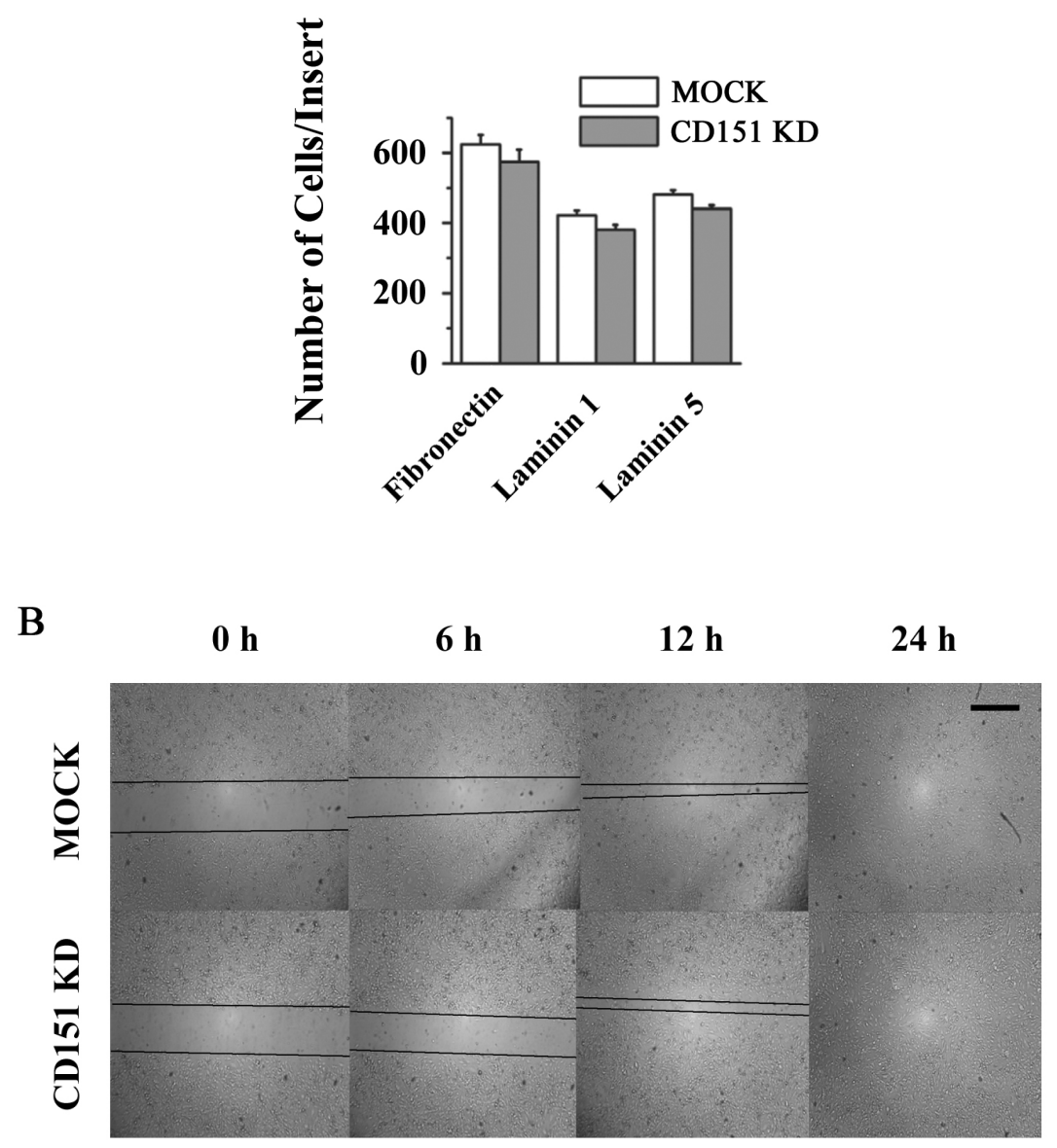

Figure 3-2. Loss of CD151 does not alter endothelial cell migration.

(A) Transwell migration assay. 2,000 HMEC-MOCK and-CD151 KD cells were placed in the transwell inserts precoated with fibronectin, laminin 1 or laminin 5, and allowed to migrate through the filters. Migrated cells were then stained and counted. Data were from 4 independent experiments. (B) Wound healing assay. HMEC transductants were cultured in 24-well plate until confluence. Then cells monolayers were starved, wounded and cultured in serum-free media. The wound area were monitored and photographed at indicated time points. $\mathrm{Bar}=400 \mu \mathrm{m}$. 
A

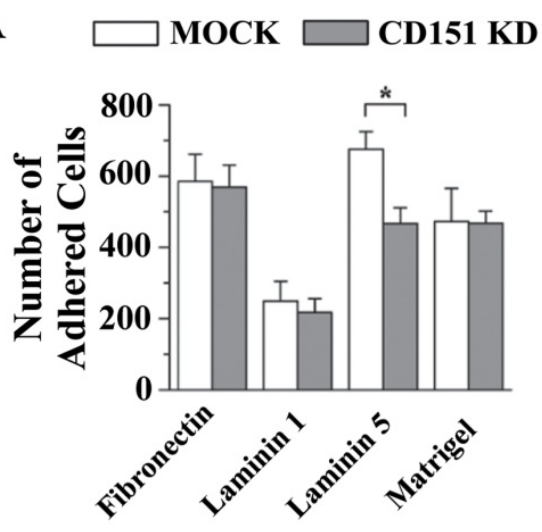

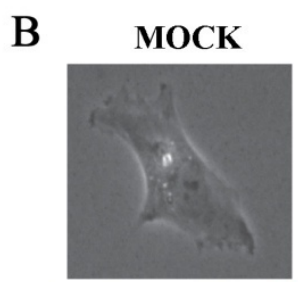

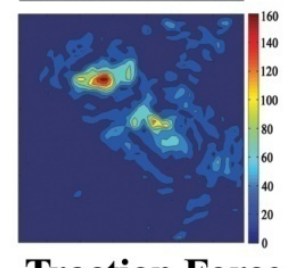

Traction Force Contour Plot

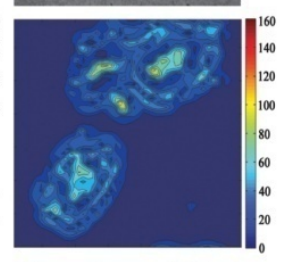

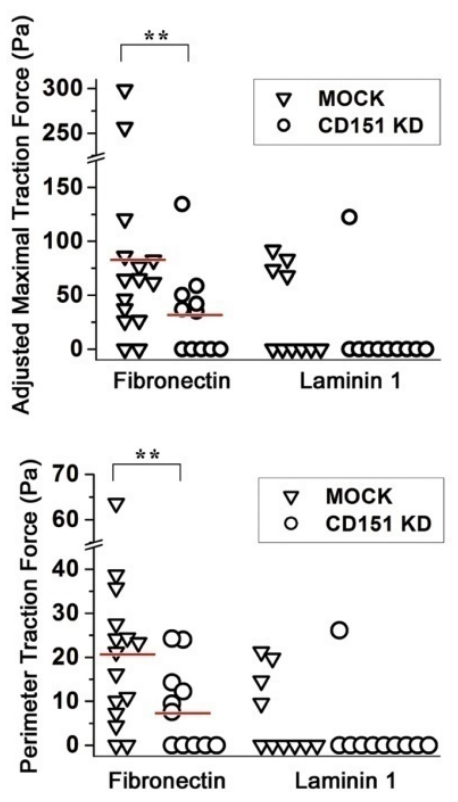

Laminin

Figure 3-3. Loss of CD151 diminishes endothelial cell-matrix adhesiveness.

(A) Cell-matrix adhesion assay. HMEC transductants were seed on ECM substrate-coated wells, and settled at $37^{\circ} \mathrm{C}$ for $35 \mathrm{~min}$. Non-adherent cells were then removed by gentle washes. The adhered cells were counted. Error bars represent standard errors from four independent experiments. ${ }^{*} \mathrm{p}<0.01$. (B) Traction force microscopy. Cells were placed on fibronectin- or laminin 1-coated fluorescent beads-embedded PAA gel. Traction forces exerted by cells cause deformation of the beaded gels. The deformation is characterized by the two-dimensional displacement of the beads and is translated into the traction field after correlation of deformed and undeformed gels. Left: phase contrast images and traction field contour plots of HMEC transductants plated on fibronectin-coated PAA gels. $B a r=10 \mu \mathrm{m}$. Right: adjusted maximum and perimeter traction forces of HMEC transductants on fibronectin and laminin $1 .{ }^{* *} \mathrm{p}<0.05$. 
underlying substrate through focal adhesion and close contacts and represents cell-matrix

adhesiveness. ${ }^{170,171}$ Cells placed on elastic substrate could cause deformations of the flexible substrate such as PAA gels due to the traction forces upon cell adhesion. ${ }^{170,171} \mathrm{By}$ detecting the levels of deformations on the fluorescence-beaded gels together with imaging and computational advances, ${ }^{170,171}$ we are able to measure traction forces and quantify the strength of cell adhesion. As shown in Figure 3-3B, compared with MOCK cells $(\mathrm{n}=15), \mathrm{CD} 151 \mathrm{KD}$ cells $(\mathrm{n}=11)$ plated on fibronectin-coated gels are significantly decreased in generation of both maximal traction forces (32.5 Pa in CD151 $\mathrm{KD}$ versus $85.6 \mathrm{~Pa}$ in $\mathrm{MOCK}$ ) and perimeter traction forces (8.3 Pa versus $20.5 \mathrm{~Pa}$ ). On laminin 1-coated gels, 6 out of 10 MOCK cells and 9 out of 10 CD151 KD cells exhibited traction stress below the threshold of measurement. Because the maximum stresses in a majority of cells are not measurable, a statistical comparison was not performed. But the undetectable traction force found in more CD151 KD cells implies that CD151 silencing leads to less traction force on laminin 1 . Hence, CD151 is needed for the strengthening of cell-matrix adhesion on both fibronectin and laminin 1.

\subsubsection{CD151 Does Not Determine Physical Properties of $\alpha 3$ Integrin and CD9 in Endothelial Cell-matrix Interface}

CD151 associates with integrins, such as integrin $\alpha 3 \beta 1$, and tetraspanins, such as CD9, in TEM. ${ }^{31}$ To determine how the loss of CD151 expression diminishes the strengthen of cell-matrix adhesion, we investigated the physical properties of TEM components integrin $\alpha 3 \beta 1$ and CD9 at the basal plasma membrane of endothelial cells using TIRF microscopy, which visualizes the molecular distribution and organization at the cell and matrix interface. As shown in Figure 3-4, at the basal cell surfaces of the majority of HMEC-MOCK and -CD151 KD cells, CD9 largely localizes at the peripheral regions whereas integrin $\alpha 3 \beta 1$ relatively evenly distributed across the pericentrolar area. The fluorescent particles of integrin $\alpha 3 \beta 1$ and CD9 exhibit no distinguishable difference in term of number, size, morphology, and distribution between HMEC-MOCK and -CD151 KD cells, suggesting that CD151 does not modulate cell-matrix adhesion by altering these biophysical properties of TEM.

\subsubsection{CD151 Regulates Glycosylation of $\alpha 3$ Integrin}

CD151 was shown to be required for glycosylation of integrin $\alpha 3 \beta 1$ in epithelial cells. ${ }^{62}$ The glycosylations at the light chain of $\alpha 3$ integrin subunit result in faster migrating rate of the protein in SDS-PAGE. ${ }^{62}$ In endothelial cell, we observed similar phenomena. As shown in Figure 3-5, in HMEC-MOCK cells, $\alpha 3$ integrin light chain resolved as closely spaced multi-bands corresponding to the glycosylated protein on the 

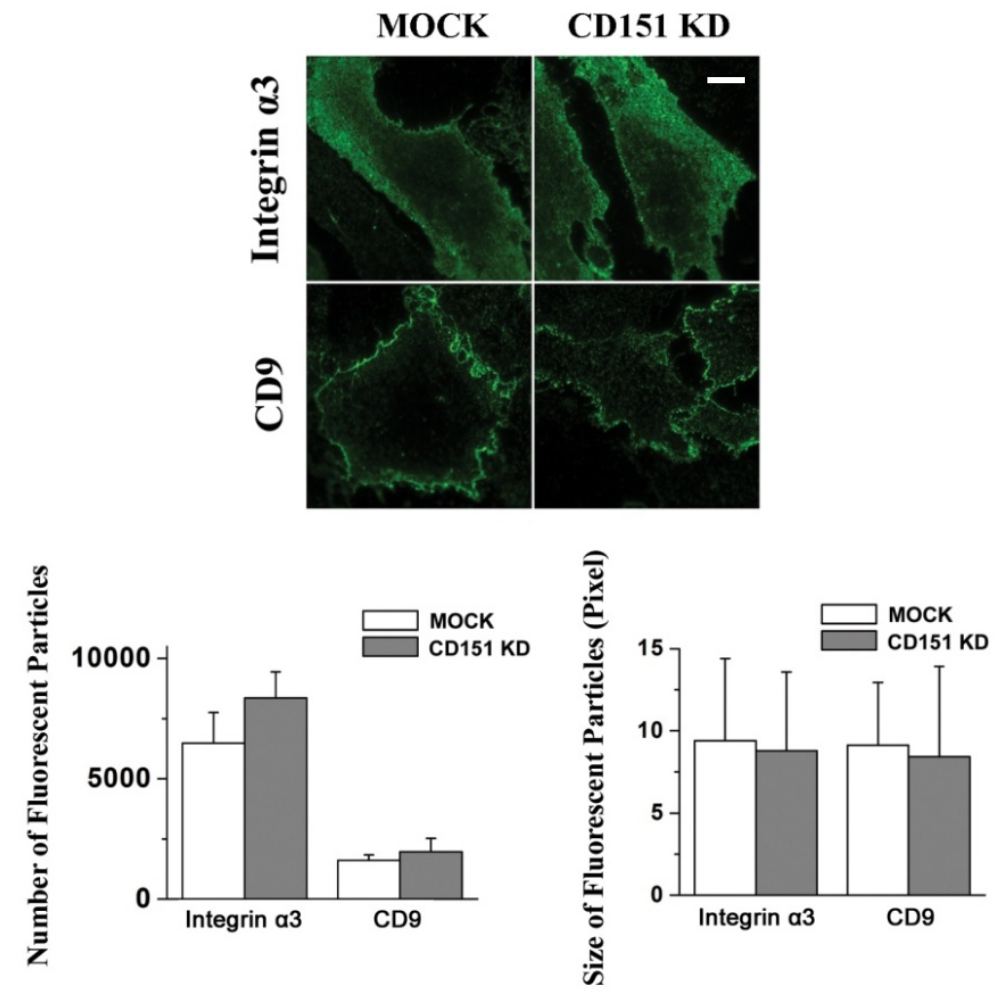

Figure 3-4. TIRF microscopy.

HMEC transductants was fixed without permeabilization, probed with $\alpha 3$ integrin or CD9 $\mathrm{mAb}$ and Alex488-conjugated secondary antibody, and visualized by a TIRF microscope. Shown are representative TIRF images (upper panel) and quantitative results of the size and numbers of fluorescent detective particles (lower panel) in individual cells $(n=20)$. Bar $=10 \mu \mathrm{m}$. 
MOCK CD151 KD

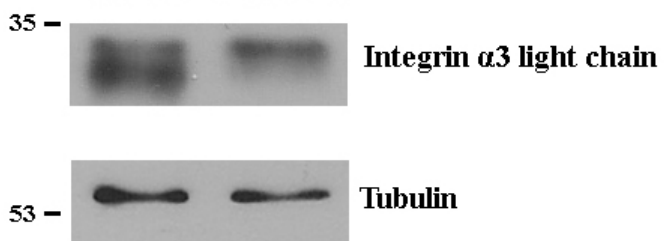

Figure 3-5. Loss of CD151 inhibits glycosylation of $\alpha 3$ integrin.

HMEC-MOCK and -CD151 KD cell lysates were resolved by 12\% SDS-PAGE under reducing conditions and analyzed by immunoblotting using specific Abs for $\alpha 3$ integrin (D23) and $\beta$-tubulin. 
lower positions and the unglycosylated form on top with equal intensity. In contrast, integrin $\alpha 3$ light chain of CD151-silenced HMECs is prominent in the top band, suggesting loss of CD151 blocks glycosylation of $\alpha 3$ integrin and showing consistence with early observation. ${ }^{62}$ This result points to a potential novel mechanism by which CD151 regulates endothelial cell-matrix adhesion strengthening, i.e. CD151 might optimize the adhesive properties of integrin $\alpha 3 \beta 1$ by modulating glycosylation of the integrin.

\subsubsection{CD151 Strengthens the Connection of $\beta 1$ Integrin to Actin Cytoskeleton}

In focal adhesions, the anchorage of integrins to actin filaments is crucial for the proper mechanical strength of cell-matrix adhesion. To evaluate the roles of CD151 in the connection of $\beta 1$ integrins with actin cytoskeleton, we compared the amounts of $\beta 1$ integrin in low concentration of Triton X-100-soluble (cytoplasmic) and -insoluble (cytoskeleton associated) fractions, and found $\beta 1$ integrin is more soluble in CD151 KD cells than in MOCK cells. As shown in Figure 3-6, CD151 KD cells contain more detergent-soluble and less detergent-insoluble $\beta 1$ integrin than MOCK cells. This result suggests loss of CD151 disturbs the association of $\beta 1$ integrin with the detergent-insoluble actin cytoskeletal matrix and supports the idea that CD151 promotes the physical and functional linkage of $\beta 1$ integrins with actin cytoskeleton for strengthening of cell-matrix adhesion.

\subsubsection{Reinforcement of Cell-matrix Adhesion Partially Rescues the Defective Vascular Network Structures Formed by CD151-silenced Endothelial Cells}

After observing that silencing of CD151 expression impairs cell adhesion onto laminin 5 and cell traction on fibronectin and laminin 1, we then examined whether the reinforcement of cell-matrix adhesion can overcome the instability of endothelial network formed by CD151 KD cells during vascular morphogenesis. We added TS2/16 to the 3D capillary formation experiment. As shown in Figure 3-7 and Table 3-1, integrin activation by $\beta 1$ integrin-activating mAb TS2/16 stabilized the network structure of CD151 KD cells to the levels of untreated and control Ab-treated MOCK cells, suggesting that $\beta 1$ integrin activation is sufficient to maintain the endothelial network in which CD151 is silenced. Notably, TS2/16-stimulated MOCK cells form a more anastomosing and more stabilized endothelial network, suggesting $\beta 1$ integrin activation cannot fully rescue the defects of CD151 silencing or optimize the network maintenance to the MOCK level. These data also indicates that the impaired cell-matrix adhesion might not be the only phenotypes in endothelial cells caused by loss of CD151. 


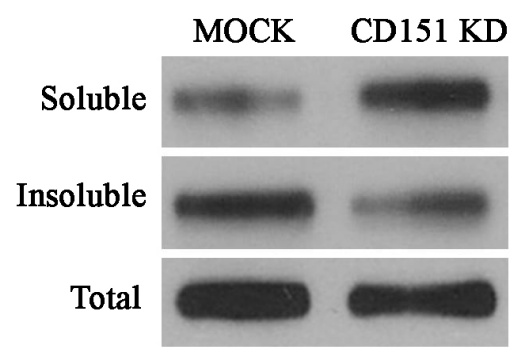

Blot: $\beta 1$ integrin

Figure 3-6. Loss of CD151 elevates detergent solubility of $\beta 1$ integrin.

HMEC transductants were lysed with $0.05 \%$ Triton X-100 in HEPES buffer. Soluble and insoluble fractions were obtained after ultracentrifugation, and were subjected to SDS-PAGE. $\beta 1$ integrin distributed in the fractions as well as whole cell lysates were then detected by immunoblotting using TS2/16 mAb. 


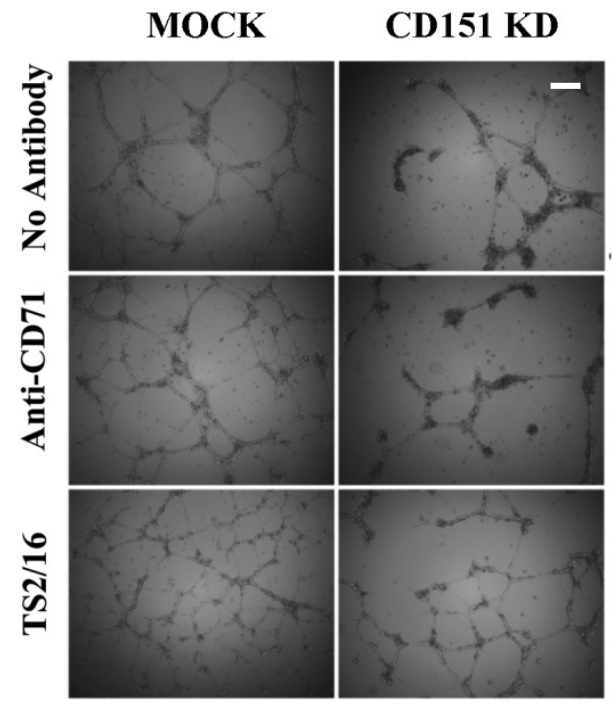

Figure 3-7. TS2/16 partially rescues the defects in maintenance of capillary structures in CD151-silenced endothelial cells.

HMEC transductants were incubated with $1 \mu \mathrm{g} / \mathrm{ml} \beta 1$ integrin activating mAb TS2/16 on ice for $30 \mathrm{~min}$, and were plated on Matrigel in the presence of TS2/16. The capillary-like structures were photographed and quantified. An isotype-matched mAb (anti-human CD71 antibody) serves as negative control. Bar $=250 \mu \mathrm{m}$.

Table 3-1. Quantitative results of Matrigel cable formation assay with TS2/16 treatment.

\begin{tabular}{llllll}
\hline Antibodies & \multicolumn{2}{l}{$\begin{array}{l}\text { Number of Circled Regions } \\
\text { per View of Field }\end{array}$} & & \multicolumn{2}{l}{$\begin{array}{l}\text { Total Cable Length per View } \\
\text { of Field }(\boldsymbol{\mu m})\end{array}$} \\
\cline { 2 - 3 } \cline { 6 - 7 } & MOCK & CD151 KD & & MOCK & CD151 KD \\
\hline $\mathrm{No} \mathrm{mAb}$ & $34.2 \pm 3.3$ & $6 \pm 1.4$ & & $13.0 \pm 0.6$ & $4.3 \pm 1.0$ \\
$\mathrm{CD} 71 \mathrm{mAb}$ & $34.5 \pm 3.5$ & $7.25 \pm 4.8$ & & $14.7 \pm 2.9$ & $5.6 \pm 3.7$ \\
$\mathrm{TS} 2 / 16$ & $74.2 \pm 6.8$ & $29.5 \pm 3.8$ & & $25.5 \pm 1.5$ & $11.0 \pm 1.4$ \\
\hline
\end{tabular}




\subsubsection{CD151 Regulates CD44 Expression in Endothelial Cells}

CD44 is encoded by an alternately spliced gene and contains tens of splicing variants. ${ }^{172}$ To determine the effect of loss of CD151 in total CD44 mRNA expression, we performed qRT-PCR used a pair of primers located at CD44 exons 1 and 2, which are expressed by all CD44 splicing transcripts. As shown in Figure 3-8C, the total mRNA level of CD44 was decreased, 1.0 fold, in CD151-silencing HMECs compared to non-silencing MOCK cells. Western blot and flow cytometry analysis confirmed both total cellular and cell surface expression of CD44 protein were significantly downregulated in CD151-silencing cells (Figure 3-8A and B). Hence we determined that loss of CD151 downregulates gene expression of CD44. Because CD44 binds to hyaluronan and connects with membrane-cytoskeleton linker ERM complex, ${ }^{173}$ CD151 likely regulates CD44-mediated but integrin-independent connection between matrix, plasma membrane, and cytoskeleton.

\subsection{Summary}

In order to fully understand the mechanisms by which CD151 regulates vascular morphogenesis and stability, we first investigated the roles of CD151 in endothelial cells and cell motility. We found that CD151 does not regulate endothelial cell death and cell motility and excluded the possibilities that CD151 promotes vascular stability by regulating endothelial cell death and cell motility.

We next demonstrated that CD151 plays important roles in endothelial cell-matrix adhesion. CD151 is needed for integrin $\alpha 3 \beta 1$-mediated initial establishment of cell adhesion on laminin 5, and integrin $\alpha 5 \beta 1$ - and $\alpha 6 \beta 1$-mediated cell adhesion strengthening on fibronectin and laminin 1. We provide evidence that CD151 is not required for the biophysical properties of $\alpha 3$ integrin- and/or CD9-containing TEM. We next demonstrated that CD151 modulate the glycosylation of $\alpha 3$ integrin and regulate the connection of $\beta 1$ integrin with cytoskeleton. In addition, reinforcing cell-matrix adhesion by activating $\beta 1$ integrin activity could rescue the defects in maintenance of vascular morphogenesis caused by loss of CD151 to some extents, evidencing that vascular stability is at least partially dependent on CD151-mediated endothelial cell-matrix adhesion. In addition, we found CD151 upregulates CD44 expression, suggesting a regulatory role of CD151 in integrin-independent cell-matrix adhesion.

In short, the data above indicate that endothelial cell-matrix adhesiveness but not cell death and cell motility become altered in the absence of CD151 and that vascular instability caused by CD151 removal can be at least partially attributed to the impaired endothelial cell-matrix adhesiveness. 
A

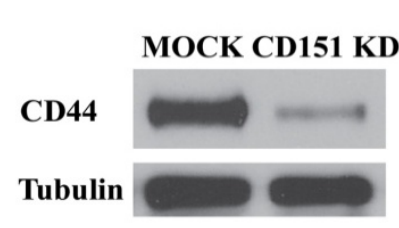

B

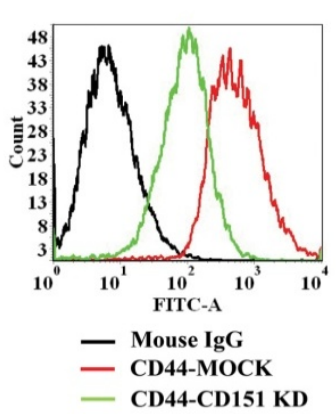

C

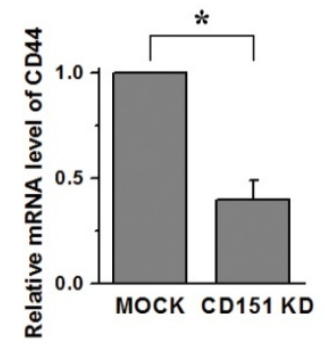

Figure 3-8. Loss of CD151 inhibits CD44 expression.

(A) Total cellular, (B) cell surface, and (C) mRNA levels of CD44 in HMEC transfectants were determined by western blot, flow cytometry and qRT-PCR, respectively. ${ }^{*} \mathrm{p}<0.01$ 


\section{CHAPTER 4. CD151 REINFORCES ENDOTHELIAL CELL-CELL ADHESION}

\subsection{Introduction}

Cell-cell adhesion is primarily mediated by cadherins, a family of calcium-dependent single pass transmembrane molecules with differentially tissue-specific expression pattern. ${ }^{120}$ At cell-cell junction sites, cadherins and $\alpha-, \beta$-, and $\gamma$-catenin, and $\mathrm{p} 120^{\mathrm{ctn}}$ physically and functionally associate into adherens junction complex to mediate homotypic intercellular interactions in the presence of calcium. ${ }^{120}$ Similar to integrins in focal adhesions, the cadherin/catenin junctional complex also associates with vinculin or $\alpha$-actinin to connect with cortical cytoskeleton. ${ }^{119,120}$ Within endothelium, the roles of VE-cadherin-mediated AJs in vascular morphogenesis and vascular barrier function have been well documented. ${ }^{12-14,174,175}$ In addition to VE-cadherin, endothelial cells also express other calcium-independent cell-cell adhesion molecules, such as occludin, claudins and JAM-1 as well as PECAM- 1 and ICAM-2. ${ }^{11,16,176}$

Because diminished endothelial cell-cell adhesion could lead to instability of newly formed vasculature, CD151 may sustain neovascularization by reinforcing cell-cell interactions. Hence, we used both in vitro and in vivo models to 1) assess the roles of CD151 in modulating endothelial cell-cell adhesiveness and maintaining the structural soundness of cell-cell contacts and 2) define the signaling steps by which CD151 stabilizes endothelial cell-cell adhesion.

\subsection{Materials and Methods}

\subsubsection{Materials}

Mouse anti-VE-cadherin mAb was from Santa Cruz Biotechnology (Santa Cruz, $\mathrm{CA}$ ). Mouse anti- $\alpha$ - and $\beta$-catenin mAbs were purchased from BD Biosciences (San Jose, CA). Mouse anti-p $120^{\text {ctn }}$ was from Sigma-Aldrich (St. Louis, MO). Protein-A \& -G Sepharose beads were purchased from GE Healthcare (Piscataway, MA). Rho kinase inhibitor Y27632 was purchased from Calbiochem (San Diego, CA). All other reagents were described in previous chapters. 


\subsubsection{Cell Aggregation Assay}

To determine the roles of CD151 in calcium-dependent and -independent cell-cell adhesion of endothelial cells, the hanging drop aggregation assay was performed as described. ${ }^{177}$ A single cell suspension with $2 \times 10^{4}$ cells in $30 \mu$ of complete cell culture media or of calcium-free media was suspended as hanging drop on the lid of a 24-well culture plate and allowed to aggregate overnight. Then the cells were subjected to shear force by passing them 10 times through a $200 \mu$ l-filter pipette tip. Cells were photographed, and 1) the number of single cells, and 2) the cells in clusters were counted using ImageJ software. Then the ratios of aggregated cells to total cells were calculated, and statistical analysis was performed. To evaluate the role of CD151 in crosstalking RhoA signaling and endothelial cell-cell adhesion, we applied $10 \mu \mathrm{M}$ Y27632 (a Rho kinase inhibitor, which will be discussed in Chapter 5) to the cell suspension and performd the hanging drop aggregation assay as described above.

\subsubsection{Analysis of Cellular Distribution Adherens Junctional Complex}

For the localization of VE-cadherin and $\beta$-catenin, HUVEC transductants were plated on glass bottom cell culture dishes and cultured for 4 days to confluence. The cell monolayer was then starved for 6 hours and processed for immunofluorescence in the presence of $2 \mathrm{mM} \mathrm{CaCl}_{2}$ using VE-cadherin and $\beta$-catenin mAbs. Distribution of VE-cadherin and $\beta$-catenin in CD151-silenced cells and MOCK cells was visualized under a confocal fluorescent microscope.

\subsubsection{Transmission Electron Microscopy (TEM)}

For TEM, 12 week-old male mice were perfused with $10 \mathrm{ml}$ of $0.1 \mathrm{M}$ sodium cacodylate buffer and fixed with $10 \mathrm{ml}$ of $2.5 \%$ glutaraldehyde in the sodium cacodylate buffer. The lung tissues were then excised and cut into approximately 1 X 1 X $1 \mathrm{~mm}^{3}$ cubes. The tissue samples were post-fixed in 1\% osmium tetroxide, dehydrated in dimethoxypropane, and embedded in epoxyresin LX-112 (Ladd Research, Williston, VT). The sections were stained with $0.3 \%$ potassium ferrocyanide in $2.0 \%$ osmium tetroxide and $4.0 \%$ uranyl acetate, and photographed under a transmission electron microscope.

\subsubsection{Analysis of Protein Association of Adherens Junctional Complex}

Protein associations within adherens junctional complex were analyzed by co-immunoprecipitation (co-IP) as described previously with modifications ${ }^{178}$ using 
VE-cadherin and $\beta$-catenin mAbs. In brief, post-confluent HMEC cells were lysed on ice for 15 min using co-IP lysis buffer (1\% Brij 97, 0.2\% Triton X-100, $50 \mathrm{mM}$ HEPES, pH $7.2,150 \mathrm{mM} \mathrm{NaCl}$ and $2 \mathrm{mM} \mathrm{CaCl} 2$ ) containing proteinase and phosphatase inhibitor cocktail $(10 \mu \mathrm{g} / \mathrm{ml}$ leupeptin, $10 \mu \mathrm{g} / \mathrm{ml}$ aprotinin, $2 \mathrm{mM}$ sodium orthovanadate, $2 \mathrm{mM}$ PMSF, $2 \mathrm{mM}$ sodium fluoride and $2 \mathrm{mM}$ sodium pyrophosphate). Insoluble fraction was removed by centrifugation at $13,000 \mathrm{rpm}$ for $10 \mathrm{~min}$. The amount of total protein in supernatant was quantified using MicroBCA protein assay kit (Pierce, Rockford, IL) and adjusted to equal. The lysates were pre-cleared with protein-A \& -G Sepharose beads 3 times with each clearance of 1 hour at $4^{\circ} \mathrm{C}$ and then incubated with specific antibody (1 $\mu \mathrm{g} \mathrm{Ab} / 500 \mu \mathrm{g}$ total protein) at $4^{\circ} \mathrm{C}$ for 1.5 hour to precipitate total cellular protein of interest, followed by another 3 hour-incubation with protein-A \& $-\mathrm{G}$ beads to bring down the precipitates. The beads were collected and washed three times in lysis buffer. The protein levels within the precipitates were then detected by immunoblotting.

\subsubsection{Detergent Solubility Assay for VE-cadherin}

The cytoskeletal association of VE-cadherin was analyzed by the detergent solubility assay. In brief, post-confluent HMEC transductants were solubilized with $0.03 \%$ or $0.15 \%$ Triton X-100 in HEPES buffer containing $2 \mathrm{mM} \mathrm{CaCl}_{2}$. Then detergent-soluble and -insoluble fractions were obtained as described in Section 3.2.7. VE-cadherin distributed in the fractions as well as total cellular VE-cadherin was detected by immunoblotting using VE-cadherin mAb.

\subsection{Results}

\subsubsection{CD151 Strengthens Calcium-dependent Cell-cell Adhesion in Endothelial Cells}

Endothelial cell-cell adhesion is critical for blood vessel formation and stability. Since CD151 promotes epithelial cell-cell adhesion, ${ }^{85,116,117}$ we predict that CD151-regulated endothelial cell-cell adhesion plays a role in CD151-dependent vasculature stability. Hence, we compared endothelial cell-cell adhesion between HMEC-MOCK and -CD151 KD cells using cell aggregation assay, a standard approach to quantify cell-cell adhesion, in the presence and absence of calcium. We observed that the loss of CD151 expression compromised calcium-dependent endothelial cell-cell adhesion (Figure 4-1). In the presence of calcium, approximately two-thirds of HMEC-MOCK cells (66.1\% of total) were aggregated in the hanging drops of cell suspension after the sheer force treatment. In contrast, a significantly less aggregation (41\% of total) was observed in CD151 KD cells. This result suggests CD151 strengthens 

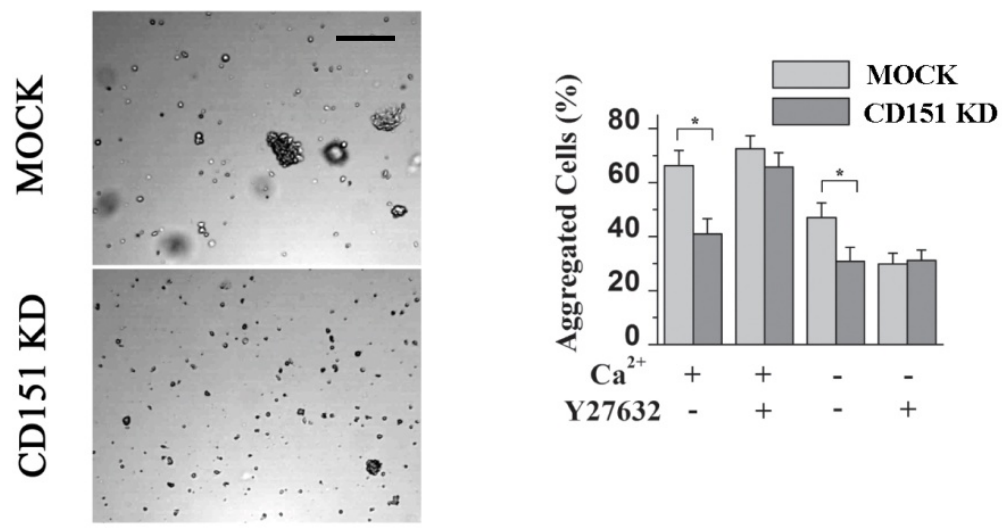

Figure 4-1. Loss of CD151 impairs endothelial cell-cell adhesiveness.

$2 \times 10^{4}$ cells were seeded into 30- $\mu$ l hanging drop cultures in absence or presence of $\mathrm{Ca}^{2+}$ and/or Rho kinase inhibitor Y27632 $(10 \mu \mathrm{M})$, which was used to determine the role of CD151 in crosstalking RhoA signaling and cell-cell adhesion in endothelial cells, to aggregate at $37^{\circ} \mathrm{C}$ overnight. After trituration by passing the cell cluster 10 times through a 200- $\mu$ l filter pipette tip, cell cluster was photographed, and the degree of dissociation of the aggregates was quantified using ImageJ analysis. Presented are representative images of cell aggregation in the presence of $\mathrm{Ca}^{2+}$ without Y27632 (left panel, bar $=100 \mu \mathrm{m}$ ) and the quantitative results (right panel). ${ }^{*} \mathrm{p}<0.01$. 
calcium-dependent cell-cell adhesion in endothelial cells.

\subsubsection{CD151 Stabilizes VE-cadherin-mediated Adherens Junctions}

The calcium-dependent cell-cell adhesion, i.e. adherens junctions, is mediated by VE-cadherin and its associated catenins. ${ }^{120}$ In endothelial cells, CD151 is colocalized with VE-cadherin at cell-cell contacts. ${ }^{80}$ Since VE-cadherin is essential for vascular morphogenesis ${ }^{12-15}$ and barrier function, ${ }^{10,11,14}$ we next determined whether CD151 promotes endothelial cell-cell adhesion by stabilizing adherens junctions. As shown in Figure 4-2, VE-cadherin and $\beta$-catenin were uniformly and continuously localized at cell-cell contacts in HUVEC-MOCK monolayer, indicating the structural soundness of endothelial adherens junctions. In HUVEC-CD151 KD cells, VE-cadherin and $\beta$-catenin distribution was irregular and discontinuous at the cell-cell contacts and also found in cytoplasma due to the loss of structural integrity in endothelial adherens junction. These results suggest CD151 plays important roles in organization of AJs, thereby providing a mechanism by which CD151 regulates to vascular stability.

To validate the in vitro observation, we examined the ultrastructure of pulmonary endothelium, which are identified by the existence of red blood cells within blood vessels, in CD151-null mice using TEM, a well-established technology to investigate cell-cell junctions. In transmission electron micrographs, endothelial cell-cell adhesion, particularly adherens junctions, is visible as narrow bands of electron-dense material flanking the adjacent cells. ${ }^{179}$ As shown in Figure 4-3, the WT mouse lung tissue shows nicely organized cell-cell junction structure in endothelium, as indicated by the continuous and closely contacted electron-dense material between endothelial cells. In contrast, many endothelial cell junctions become loose, and/or less electronically dense in CD151-null mice under electron microscopy. Two adjacent plasma membranes in endothelial cell junctions tend to dissociate from each other and form open gaps between them when CD151 is ablated. This phenotype supports the decreased cell-cell adhesion and increased permeability as observed above.

\subsubsection{CD151 Is Not Required for the Assembly of Adherens Junctional Complexes in Endothelial Cells}

It was reported that integrin $\alpha 3 \beta 1$ and CD151 enhance cell-cell adhesion by stabilizing cadherin-catenin complex in epithelial cells. ${ }^{116}$ To explore the in-depth mechanism by which CD151 strengthens endothelial cell-cell junctions, we further examined whether CD151 enhances cell-cell adhesion by stabilizing VE-cadherin junctional complex and/or the connection of this complex to actin cytoskeleton. As 


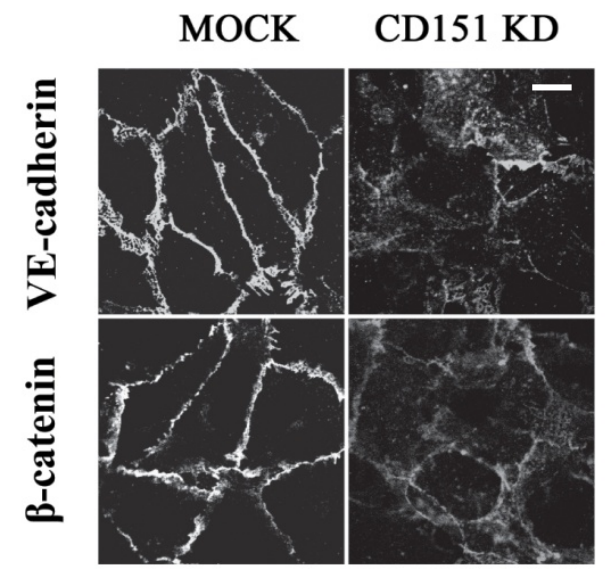

Figure 4-2. Adherens junctional complexes are mis-localized in CD151-silenced endothelial cell monolayer.

HUVEC transductants were cultured for 4 days to confluence. After 6-hour starvation, HUVEC monolayers were fixed, permeablized and stained with specific antibodies. Then the distributions of VE-cadherin and $\beta$-catenin in the monolayers were visualized under a confocal microscope. Bar $=10 \mu \mathrm{m}$.

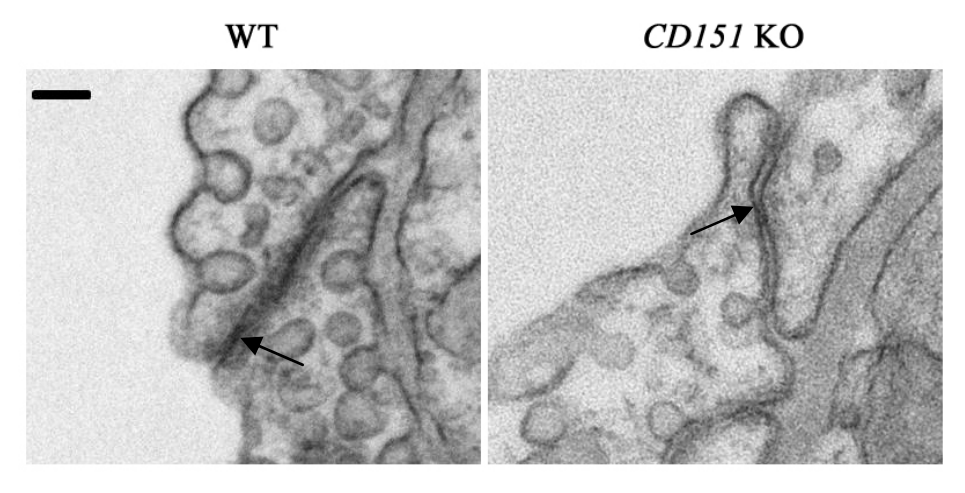

Figure 4-3. Abnormal endothelial cell-cell adhesion in lung vessels of CD151 KO mice as seen in electron micrographs.

CD151 KO and littermate WT mice were perfused and fixed with $2.5 \%$ glutaraldehyde. Then mouse lung tissues were excised and processed for TEM. Bar $=100 \mathrm{~nm}$. 
revealed in co-immunoprecipitation, the levels of $\alpha$-, $\beta$-catenin and $\mathrm{p} 120^{\mathrm{ctn}}$ associated with VE-cadherin and the levels of VE-cadherin, $\alpha$-catenin and $\mathrm{p} 120^{\mathrm{ctn}}$ associated with $\beta$-catenin remained unchanged in HMEC-MOCK and -CD151 KD cells (Figure 4-4), suggesting that CD151 is not required for and also probably does not modulate the assembly of VE-cadherin junctional complex.

\subsubsection{CD151 Reinforces the Cytoskeletal Connection of VE-cadherin}

As revealed in the detergent solubility assay (Figure 4-5), more VE-cadherin was solubilized by low concentration $(0.15 \%$ or $0.03 \%)$ of Triton X-100 and found in the soluble fractions from CD151 KD cells than those from MOCK cells, while the levels of total cellular VE-cadherin remained the same in both MOCK and CD151 KD cells, suggesting loss of CD151 elevates endothelial cellular solubility. The high solubility indicates that loss of CD151 resulted in disconnection between VE-cadherin and the detergent-insoluble actin cytoskeleton matrix. Thus we demonstrated that CD151 regulates endothelial adherens junctions through enforcing the anchorage of actin filament to the junctional complex.

\subsubsection{CD151 Regulates VE-cadherin-independent Cell-cell Adhesiveness in Endothelial Cells}

Finally, from cell aggregation analysis (Figure 4-1), we observed that CD151 silencing markedly reduced endothelial cell-cell adhesion (MOCK 47\% vs. CD151 KD $30.8 \%$ ) even in calcium-depleted medium, strongly suggesting that CD151 also modulate the cell-cell adhesion mediated by calcium-independent, non-cadherin adhesion molecules, such as PECAM-1 and ICAM-2, in endothelial cells.

\subsection{Summary}

In this chapter, we focus on the roles of CD151 in endothelial cell-cell adhesion. We observed loss of CD151 attenuates endothelial cell-cell adhesiveness. This is in particular applied to adherens junctions since CD151 silencing disturbs the enriched

distribution VE-cadherin and $\beta$-catenin at cell-cell contact sites. Furthermore, CD151 is not required for the assembly of the junctional complex but rather important for the anchorage between AJ complex and cortical cytoskeleton. 


\section{IP: VE-cadherin $\quad \beta$-catenin}

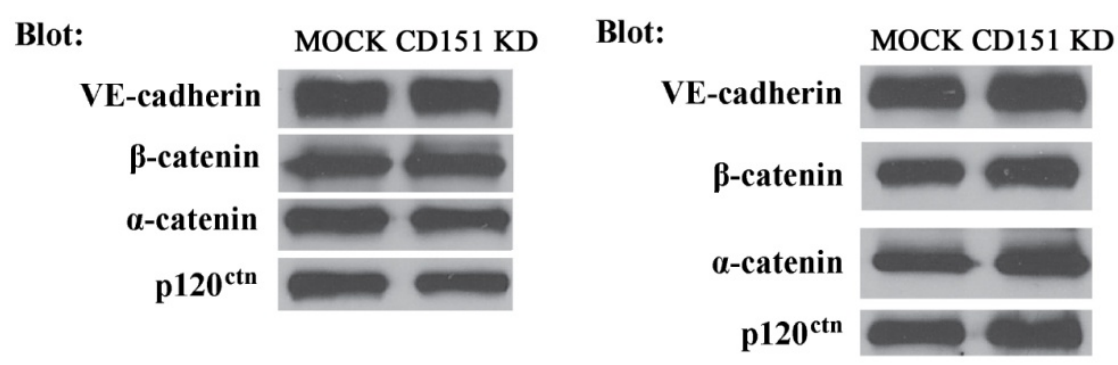

Figure 4-4. The protein association in adherens junctional complex is not affected by loss of CD151.

HMEC-MOCK or -CD151 cells were lysed in co-IP buffer. Then the indicated proteins were immunoprecipitated, followed by immunoblotting with specific antibodies. 


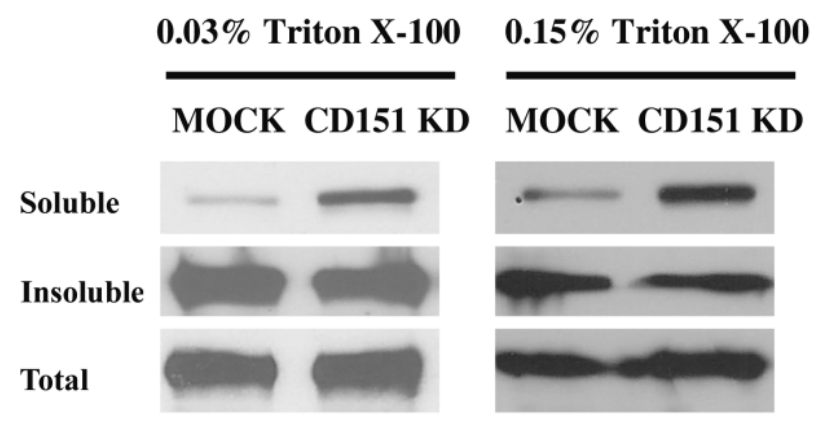

Blot: VE-cadherin

Figure 4-5. Detergent solubility assay of VE-cadherin.

HMEC-MOCK or -CD151 KD cells were solubilized with $0.03 \%$ or $0.15 \%$ Triton X-100 in HEPES. After ultracentrifugation, supernatants were used as soluble fractions, and pellets were solubilized in $1 \mathrm{X}$ SDS sample buffer and used as insoluble fractions. The fractions were then subjected to SDS-PAGE, followed by immunoblotting with anti-VE-cadherin mAb. Total cellular VE-cadherin was from 1 X SDS sample buffer extracted whole cell lysates. 


\section{CHAPTER 5. CD151 MODULATES SMALL GTPASES RAC1 AND RHOA SIGNALINGS AND RESTRAINS ENDOTHELIAL CYTOSKELTAL TENSION}

\subsection{Introduction}

Rho GTPases are a family of GTP-binding proteins, of which RhoA, Rac1 and Cdc42 are best understood. Active RhoA activates its downstream signal molecule Rho kinase and promotes serine- and threonine-phoralyation of myosin light chain (MLC) which in turn induces stress fiber formation and cytoskeletal tension (Figure 5-1). ${ }^{17}$ Previous studies have demonstrated that, RhoA-dependent cytoskeletal organization is crucial in the maintenance of endothelium stability. ${ }^{19-21}$ Excess RhoA activity disturbs the barrier function of angiogenic vessels and selectively induces regression of vasculature, whereas suppression of RhoA signaling promotes angiogenesis and vessel stability. ${ }^{19-21}$ Other small GTPases, such as Rac1, are also important in angiogenesis because Rac1-dependent cytoskeleton reorganizations could largely promote endothelial cell-cell junctions and maintain vascular barrier function. ${ }^{22,23}$ In addition, antagonism constantly exists between Rac1 and RhoA signalings, as illustrated by Bar-Sagi pathway. ${ }^{180}$

Three classes of regulatory proteins are involved in direct regulation of small GTPases activity by controlling the loading and hydrolysis of GTP: guanine nucleotide exchange factors (GEFs), GTPase-activating proteins (GAPs) and the guanine nucleotide dissociation inhibitor (GDIs), and each consists of tens of members with different specificities to Rho family. ${ }^{181-183}$ Among these regulators, p190Rho ${ }^{\text {GAP }}$, which can be avtivated by integrin signaling, ${ }^{184-186}$ and $\mathrm{p} 115 \mathrm{Rho}{ }^{\mathrm{GEF}}$, which can be activated by G-protein coupled receptors (GPCRs), ${ }^{187,188}$ are of particular importance in regulating RhoA signaling (Figure 5-1). In addition, cyclic AMP (cAMP) directly or indirectly controls small GTPase activites through protein kinase A (PKA)-mediated phosphorylation. For example, PKA suppresses RhoA activities via direct phosphorylation of RhoA, ${ }^{189,190}$ whereas Rac 1 activity can be induced by PKA-mediated phosphorylation/activation of Tiam1, a Rac1-specific GEF. ${ }^{191,192}$

Previous studies suggest that CD151 regulates Rho GTPase activities in tumor cells. ${ }^{85,112,117}$ We predict that vascular endothelial CD151 also regulates RhoA and Rac1 signalings. Therefore, we performed signal transduction studies to investigate the roles of CD151 in RhoA and Rac1 activation and their upstream and downstream signaling events. We also used the RhoA signaling inhibitors and retrovirus-based Rac1 overexpression system in the in vitro vascular morphogenesis assay to determine if CD151 regulates vascular stability via RhoA and Rac1. 


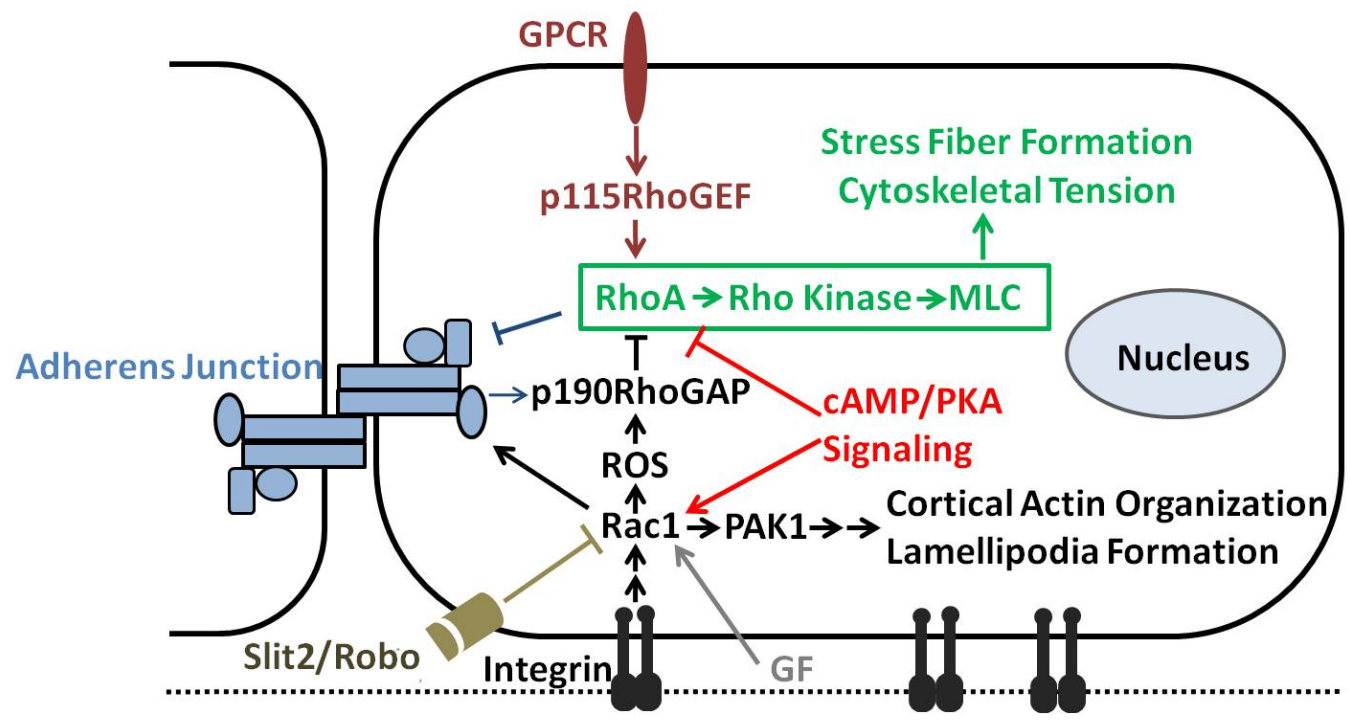

Figure 5-1. A diagram showing RhoA and Rac1 signalings and the upstream signals. Small GTPase RhoA and Rac1 are constantly antagonisic. RhoA, in its GTP-loaded form, triggers the downstream signaling cascade including activation of Rho kinase and phosphorylation of MLC, which in turn induces the assembly of actomyosin and formation of contracile actin stress fibers. Whereas Rac1, in response to GF or integrin signaling, activates PAK1 and leads to cortical actin meshwork and lamellipodia formations. The establishment of cortical actin cytoskeleton promotes the assembly of adherens junctional complexes, which recruit $\mathrm{p} 190 \mathrm{Rho}{ }^{\mathrm{GAP}}$ to the junctional sites and subsequently suppress RhoA activity to maintain the connection of adherens junctional complex with cortical actin filament. In addition, the antagonism between Rac1 and RhoA signalings could be mediated by the signaling pathway involving release of ROS and activations of eNOS and p190Rho ${ }^{\mathrm{GAP}}$. The cAMP/PKA signaling pathway promotes Rac1 activity and suppresss RhoA activity via phosphorylation, and thus provides another mechanism to maintain the antagonism between Rac1 and RhoA signalings. Other signals controlling RhoA activation also exist, such as the RhoA-specific p115Rho ${ }^{\mathrm{GEF}}$. cAMP, cyclic adenosine monophosphate; eNOS, endothelial nitric oxide synthase; GAP, GTPase-activating protein; GEF, guanine nucleotide exchange factor; GF, growth factor; GPCR, G-protein coupled receptor; MLC, myosin light chain; PAK1, p21-activating kinase 1; PKA, protein kinase A; Robo, Roundabout; ROS, reactive oxygen species. 


\subsection{Materials and Methods}

\subsubsection{Materials}

Rabbit anti-diphospho-myosin light chain (2P-MLC) II, anti-MLC II and anti-p115Rho ${ }^{\text {GEF }}$ polyclonal antibodies were obtained from Cell Signaling Technology (Beverly, MA). Mouse anti-phospho-tyrosine (PY99) monoclonal antibodies were from Santa Cruz Biotechnology (Santa Cruz, CA). Mouse anti-RhoA, anti-Rac1 and anti-p190Rho ${ }^{\text {GAP }}$ mAbs were purchased from BD Biosciences (San Jose, CA). Glutathione-conjugated Sepharose 4B beads were purchased from GE Healthcare (Piscataway, MA). Cell-permeable C3 transferase was purchased from Cytoskeleton Corp. (Denver, CO). Rho kinase inhibitor Y27632 and Adenosine-8-bromo-3', 5'- cyclic monophosphate (8-Br-cAMP) were purchased from Calbiochem (San Diego, CA). Blebbistatin and ML7 were obtained from TRC (Toronto, Canada). All other reagents were described in Chapters 2-4.

\subsubsection{Analysis of Stress Fiber Formation}

Stress fiber formation in HMEC transductants was detected by staining with Alexa Fluro 594-conjugated phalloidin.

\subsubsection{Glutathione S-transferase (GST) Pull-down Assays}

The activation of RhoA, Rac1, p115Rho ${ }^{\text {GEF }}$ or p190Rho ${ }^{\text {GAP }}$ was detected by the GST pull-down method using recombinant proteins: GST-Rhotekin Rho-binding domain (GST-TRBD) which binds to RhoAGTP, GST-p21-activated kinase 1 (PAK1) Rac1-binding domain (GST-PRBD) which binds to Rac1GTP, GST-RhoA nucleotide-empty mutant RhoA (G17A) which binds to active p115Rho ${ }^{\mathrm{GEF}}$, and GST-RhoA constitutively active mutant RhoA (Q63L) which binds to active p190Rho ${ }^{\text {GAP }}$ as described previously. ${ }^{28,193}$ To produce GST fusion proteins, $10 \mu \mathrm{l}$. coli transformed by GST fusion constructs was added into $3 \mathrm{ml} \mathrm{LB}$ media containing $100 \mu \mathrm{g} / \mathrm{ml}$ ampicillin and grew for 3 hour at $37^{\circ} \mathrm{C}$. Then the bacterial media was pipetted into $250 \mathrm{ml}$ $\mathrm{LB} /$ ampicillin and grew at $37^{\circ} \mathrm{C}$ with agitation until the absorbance at $600 \mathrm{~nm}$ was 1.0 . Then IPTG was added at $0.1 \mathrm{mM}$ final concentration into bacteria culture followed by further 2 hour-incubation at $28^{\circ} \mathrm{C}$ to induce fusion protein expression. Bacteria culture was spilt into $50 \mathrm{ml}$ aliquots and centrifuged for $30 \mathrm{~min}$ at 3,000 rpm. To generate fusion protein-bound beads, pellet bacteria from $50 \mathrm{ml}$ bacteria culture was resuspended in $2 \mathrm{ml}$ PBS and lysed by adding $80 \mu \mathrm{l}$ of $100 \mathrm{mg} / \mathrm{ml}$ lysozyme, $20 \mu \mathrm{l}$ of $1 \mathrm{M}$ DTT and $40 \mu \mathrm{l}$ of 
0.1 M proteinase inhibitor PMSF for $15 \mathrm{~min}$ on ice. Then $225 \mu \mathrm{l}$ of $10 \%$ Triton X-100, 50 $\mu l$ of $1 \mathrm{mg} / \mathrm{ml}$ DNase and $25 \mu \mathrm{l}$ of $1 \mathrm{M} \mathrm{MgCl}_{2}$ were added into bacteria lysate. After another 15 min-incubation on ice, the bacteria debris was removed by centrifugation at $10,000 \mathrm{rpm}$ for $10 \mathrm{~min}$ at $4^{\circ} \mathrm{C}$. Supernant containing GST fusion protein was incubated with $250 \mu \mathrm{l}$ glutathione-sepharose $4 \mathrm{~B}$ beads for $30 \mathrm{~min}$ at $4^{\circ} \mathrm{C}$. After 3 washes, the fusion protein-bound beads were then resuspended in PBS containing $10 \mathrm{mM}$ DTT to a $50 \%$ slurry, aliquoted and stored at $-80^{\circ} \mathrm{C}$ until needed. Prior to pulldown experiment, HMECs $\left(2.5 \times 10^{6}\right.$ cells $)$ were seeded in $10-\mathrm{cm}$ cell culture dish precoated with diluted Matrigel, and cultured at $37^{\circ} \mathrm{C}$ overnight. On the second day, cells were lysed in $1.5 \mathrm{ml}$ modified RIPA buffer (50 mM Tris, pH7.2, $500 \mathrm{mM} \mathrm{NaCl}, 1 \%$ Triton X-100, 0.5\% sodium deoxycholate, $0.1 \% \mathrm{SDS}, 10 \mathrm{mM} \mathrm{MgCl}_{2}$ ) containing protease and phosphatase inhibitor cocktail. Insoluble fraction was removed by centrifugation, and supernatant was incubated with $30 \mu \mathrm{l}$ of $50 \%$ GST fusion protein-bound bead slurry for $30 \mathrm{~min}$ at $4^{\circ} \mathrm{C}$. Then the beads were washed by washing buffer $(50 \mathrm{mM}$ Tris $\mathrm{pH} 7.2,1 \%$ Triton $\mathrm{X}-100$, $\left.150 \mathrm{mM} \mathrm{NaCl}, 10 \mathrm{mM} \mathrm{MgCl}_{2}\right) 3$ times. The precipitated proteins were eluted by Laemmli buffer and subjected to SDS-PAGE and immunoblotting using appropriate mAbs.

\subsubsection{Detection of Rho Kinase Activity}

The Rho kinase activity was measured in ELISA using a commercial available Rho kinase activity Kit (MBL International, Woburn, MA) according to the manufacture's instruction. In brief, HMEC cell lysates were prepared as in the pulldown experiments. $10 \mu \mathrm{l}$ cell lysate diluted with $90 \mu \mathrm{l}$ kinase reaction buffer (ATP plus), in triplicate, was added into 96-well plate precoated with a substrate corresponding to recombinant the C-terminus of MBS (myosin-binding subunit of myosin phosphatase), in which a threonine residue (Thr696) can be phosphorylated by Rho kinase. After 30-min incubation at $30^{\circ} \mathrm{C}$ to allow phosphorylation at the threonine-696, the wells were washed and incubated with $100 \mu$ detection solution containing HRP-conjugated AF20, an anti-phospho-MBS threonine-696 specific antibody, to bind the phosphorylated substrate with AF20. The bound HRP-conjugated AF20 catalyzed the conversion of the chromogenic substrate tetra-methlybenzidine (TMB) from a colorless solution to a blue solution, which was then converted to yellow after the addition of $100 \mu$ of $0.5 \mathrm{~N} \mathrm{H}_{2} \mathrm{SO}_{4}$ for stopping the reaction. The yellow color was then quantified by spectrophometry at the wavelength of $450 \mathrm{~nm}$. Purified recombinant Rho kinase-II positive control $(1 \mathrm{mU} / \mu \mathrm{l}$ stock) at various doses was used to calculate the enzyme catalytic domain (standard curve). The relative enzymatic activities from different HMEC transfectants were calculated using the standard curve, and compared statistically. In addition, the activity of purified recombinant Rho kinase-II (10 mU/reaction) in the presence of $10 \mu \mathrm{M}$ Y 27632 were measured by this assay in order to determine the inhibitory effect of Y27632 on Rho 
kinase.

\subsubsection{Analysis of MLC Phosphorylation}

HMEC cell lysates were prepared as in the GST pull-down assay and processed for immunoblotting to determine the levels of dual-phosphorylated MLC (2P-MLC) as well as total MLC using the specific antibodies against 2P-MLC and MLC.

\subsubsection{RhoA Signaling Inhibitor Treatment}

HMEC transfectants or MLECs were plated on Matrigel as described in Section 2.2.6. 3 hours later, C3 transferase $(2.5 \mu \mathrm{g} / \mathrm{ml})$, Y27632 $(10 \mu \mathrm{M})$ or Blebbistatin $(5 \mu \mathrm{M})$, specific inhibitor for RhoA, Rho kinase, and myosin II, respectively, was applied to cells. The cable network structures were monitored 18 hour later. ML7, a specific inhibitor against myosin light chain kinase (MLCK), served as negative control.

\subsubsection{Analysis of Upstream Signaling of RhoA and Rac1}

The tyrosine phosphorylation of p190Rho ${ }^{\text {GAP }}$ was analyzed by IP. Total cellular p190Rho ${ }^{\text {GAP }}$ in HMEC transductants was immunoprecipitated with a p190Rho ${ }^{\text {GAP }}$ mAb, and subjected to SDS-PAGE and immunoblotting. The level of tyrosine phosphorylation in the precipitated p190Rho ${ }^{\mathrm{GAP}}$ was detected by an anti-phospho-tyrosine mAb (PY99). After stripping, total input of p190Rho ${ }^{\text {GAP }}$ was then determined by p190Rho $^{\text {GAP }}$ mAb.

For fast-cycling mutant Rac1 (F28L) retroviral transduction, HMEC-MOCK or -CD151 KD cells were cultured at $37^{\circ} \mathrm{C}$ overnight and then incubated with GFP-Rac1 F28L virus in the presence of $5 \mu \mathrm{g} / \mathrm{ml}$ polybrene. On the second day, virus-containing medium was replaced by fresh medium. Rac1 F28L expressing cells were determined by flow cytometry and then enriched by GFP sorting. The Rac1 F28L-expressing cells were then used for the 3D capillary formation on Matrigel as described in Section 2.2.6. Various concentrations of 8-Br-cAMP were also used in the 3D capillary formation to explore the upstream signaling pathway.

The expression levels of Slit2 in HMEC transductants were measured by qRT-PCR as described for CD44 in Section 3.2.9. A 73 nt amplicon of human Slit2 gene was obtained from qRT-PCR using the primer pair "GTGTTCGTGCCAGCTATGAD" and "CCATCATTGATTGTCTCCACAC" for the forward and reverse primer, respectively. And the mRNA expression levels of Slits were calculated, normalized by 
setting the relative Slit2 mRNA levels in MOCK cells as 1, and compared.

\subsection{Results}

\subsubsection{CD151 Restrains Stress Fiber Formation in Endothelial Cells}

The altered endothelial cell adhesiveness drove us to analyze the effect of CD151 removal on cytoskeleton organization. CD151-silenced endothelial cells typically exhibit fibroblast-like morphology with long and thick F-actin bundles which transverse from one end to the other end of a cell (Figure 5-2), indicating that CD151 expression is needed for inhibiting robust formation of stress fibers. In contrast, CD151-expressing endothelial cells often display relatively thin, short, and intersected F-actin filaments which frequently form cortical meshwork, implying the existence of lamellipodia (Figure $5-2)$.

\subsubsection{CD151 Balances the Activations of Rac1 and RhoA Signalings}

RhoA-Rho kinase-myosin signaling cascade is primarily responsible for stress fiber formation and cell retraction, ${ }^{181}$ while the activation of Rac1 leads to the formation of cortical actin meshwork and lamellipodia. Based on the results from time-lapse microscopy and IF, we also predict that CD151 silencing enhances cell tension or contractility. By comparing the levels of active RhoA (GTP-bound RhoA) between HMEC-CD151 KD and -MOCK cells, we found that loss of CD151 results in significant upregulation of RhoA activity (Figure 5-3A, left panel). The quantitative data (Figure 5-3A, right panel) suggest that the ratio of active RhoA to total RhoA in CD151 KD cells is higher $(\sim 1$ fold $)$ than that in MOCK cells. This result underlines that CD151 is needed for restraining RhoA activity in endothelial cells. To substantiate this observation, we also measured and compared the activities of RhoA downstream such as Rho kinase activity and phosphorylation levels of MLC in HMEC-MOCK and -CD151 KD transfectants. Rho kinase activity is approximately 1-fold higher in CD151 KD cells than MOCK cells (Figure 5-3B), in accordance with elevated RhoA activation. The dual-phosphorylation of MLC at Thr18 and Ser19 (2P-MLC), which is the active form of MLC and essential for assembly of actomyosin and stress fiber formation, was also markedly increased (Figure 5-3C).

In contrast, the level of active, GTP-loaded Rac1 is reduced in HMEC-CD151 KD cells compared to -MOCK cells (Figure 5-3D). Hence, CD151 silencing deregulates the activities of Rho small GTPases and leads to the upregulation of RhoA and 


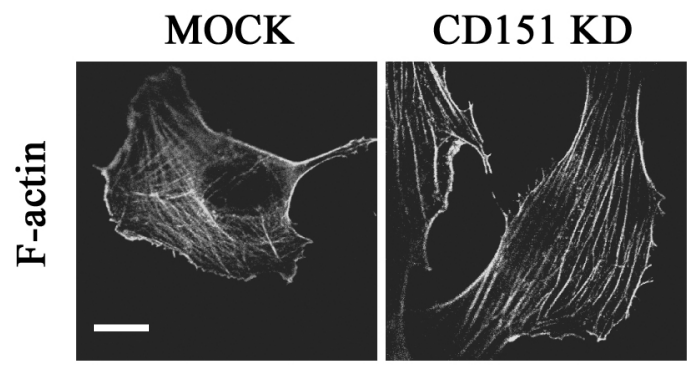

Figure 5-2. Loss of CD151 expression results in increased stress fiber formation. HMEC transductants were stained with Alex Fluor 488-conjugated phalloidin after spreading overnight. F-actin staining was then visualized with a confocal microscope. Bar $=10 \mu \mathrm{m}$. 
A

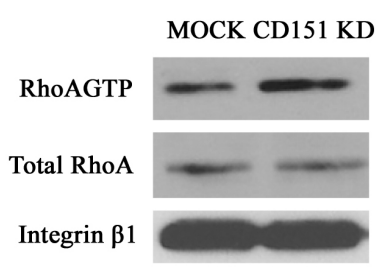

B

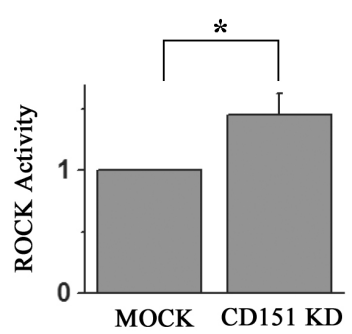

D

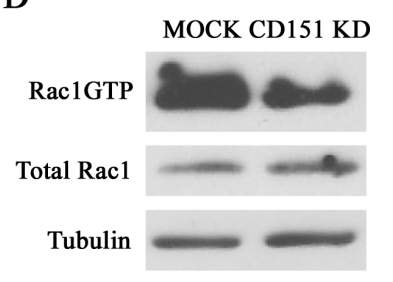

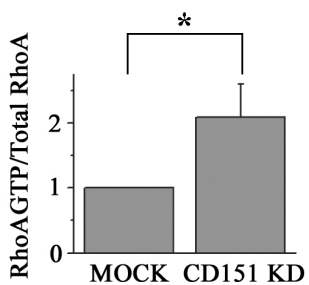

C
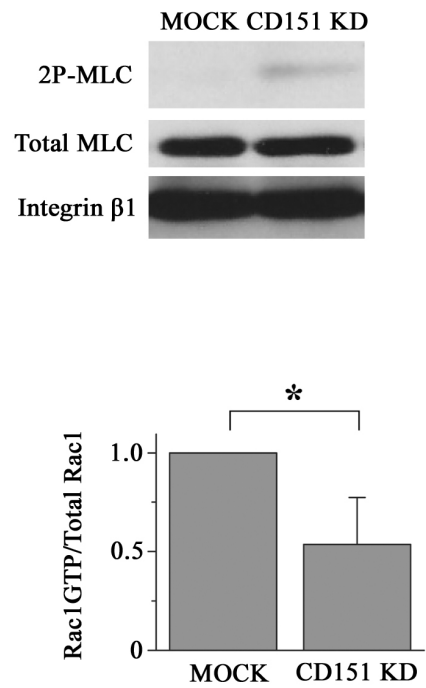

Figure 5-3. Loss of CD151 expression results in upregulation of RhoA-Rho kinase-myosin signaling and downregulation of Rac1 signaling.

(A) HMEC transductants were seeded in diluted Matrigel-coated dish and cultured overnight. Cell lysates were incubated with GST-TRBD beads to pull down GTP-bound RhoA. RhoA levels from the pulled down precipitates and in whole cell lysates were then determined by SDS-PAGE and immunoblotting using anti-human Rho mAb. The blot (left panel) is representative of four independent experiments. RhoAGTP/total RhoA ratio (right panel) was calculated from the density of the blots and normalized to MOCK group. ${ }^{*} \mathrm{p}<0.01$. (B) Cellular Rho kinase enzymatic activities were measured using a commercially available immunoassay kit. Data from 4 independent experiments were normalized to MOCK group. ${ }^{*} \mathrm{p}<0.01$. (C) HMEC cells were seeded as in RhoA pulldown assay and lysed in RIPA buffer for $20 \mathrm{~min}$ at $4^{\circ} \mathrm{C}$. After centrifugation, cell lysates were obtained and processed for SDS-PAGE and immunoblotting to detect total MLC and 2P-MLC. (D) The GTP-bound Rac1 was precipitated from HMEC cell lysates using GST-PRBD beads. Levels of Rac1GTP and total cellular Rac1 were determined by immunoblotting using Rac1 mAb (left panel). Rac1GTP/total Rac1 ratio was then calculated, normalized and compared between MOCK and CD151 KD cells (right panel). $* \mathrm{p}<0.01$. 
downregulation of Rac1 signaling, likely reflecting the antagonism between RhoA and Rac1 described earlier. ${ }^{180}$

\subsubsection{RhoA Signaling Inhibitors Restore the Unstabilization of Vascular Network Structures Caused by Loss of CD151}

The RhoA-Rho kinase-myosin signaling can be selectively blocked by the inhibitors such as C3 transferase, Y27632, and Blebbistatin, specific inhibitor for RhoA, Rho kinase, and myosin light chain assembly, respectively. ${ }^{181,194,195}$ To determine if RhoA signaling is needed for CD151-dependent maintenance of vascular stability, we applied these specific inhibitors in the in vitro vascular cable network formation assay on Matrigel. As shown in Figure 5-4A, all RhoA signaling inhibitors stabilized the endothelial network structures formed by CD151 KD cells, and MOCK and CD151 KD endothelial cells exhibited similar levels of morphogenesis. Consistently, Rho kinase inhibitor Y27632 also overrode the difference in vascular morphogenesis of primary lung endothelial cells isolated from WT and CD151-null mice (Figure 5-4B). These results underscore that sustained RhoA signaling is necessary for CD151-mediated maintenance of vascular stability. As negative control, ML-7, a specific inhibitor of myosin light chain kinase (MLCK), exerted no significant influence on the formation and maintenance of endothelial networks (Figure 5-4A). Since MLCK is exclusively involved in $\mathrm{Ca}^{2+}$-induced muscle cell contraction, ML-7 should not affect RhoA signaling in endothelial cells as predicted. In addition, to validate the rescuing effect, we show that Y27632 at $(10 \mu \mathrm{M})$ is capable of inhibiting the activity of purified Rho kinase II to a significant low level (Figure 5-5), and thus confirmed the biochemical property of Y27632 to inhibit Rho kinase activity in our system.

In addition, the impaired cell-cell adhesiveness of CD151-silenced endothelial cells was also reversed by Y27632 in the presence as well as absence of $\mathrm{Ca}^{2+}$ (Figure 4-1), indicating that RhoA signaling is essential for CD151-reinforced endothelial cell-cell adhesiveness.

\subsubsection{Elevation in Intracellular cAMP but Not Active Rac1 Can Rescue Unstabilized Vascular Network Structures Formed in CD151 KD Cells}

As a key signaling molecule important in many biological processes, cAMP has a well-established protective effect on endothelial barrier properties. ${ }^{196-200}$ CAMP, through activation of PKA-dependent phosphorylation on Rho GTPases, could enhance vascular barrier by inducing Rac1-mediated cytoskeletal reorganization and/or inhibiting RhoA activation. ${ }^{196-200}$ To investigate the role of cAMP signaling in CD151-mediated 
A

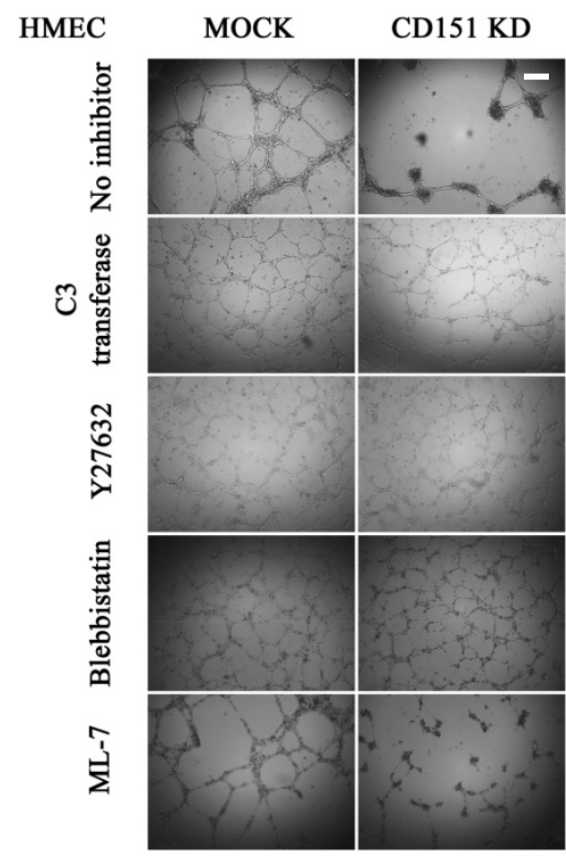

B

MLEC

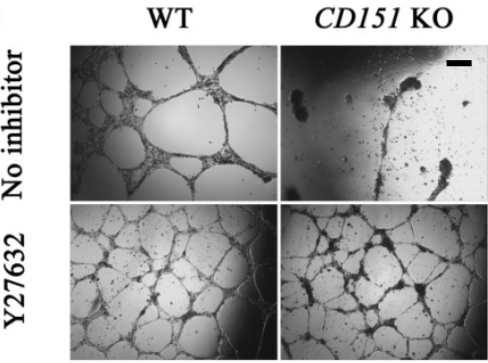

Figure 5-4. Rho signaling inhibitors restore the defective vascular network structures caused by loss of CD151.

For the in vitro cable network formation assay, various inhibitors (C3 transferase, 2.5 $\mu \mathrm{g} / \mathrm{ml}$; Y27632, $10 \mu \mathrm{M}$; Blebbistatin, $5 \mu \mathrm{M}$; and ML-7, $5 \mu \mathrm{M}$ ) were added at 4 hour after HMEC transductants (A) or MLECs (B) were plated on Matrigel. The capillary-like structures were photographed 14 hour later. Bar $=250 \mu \mathrm{m}$. 


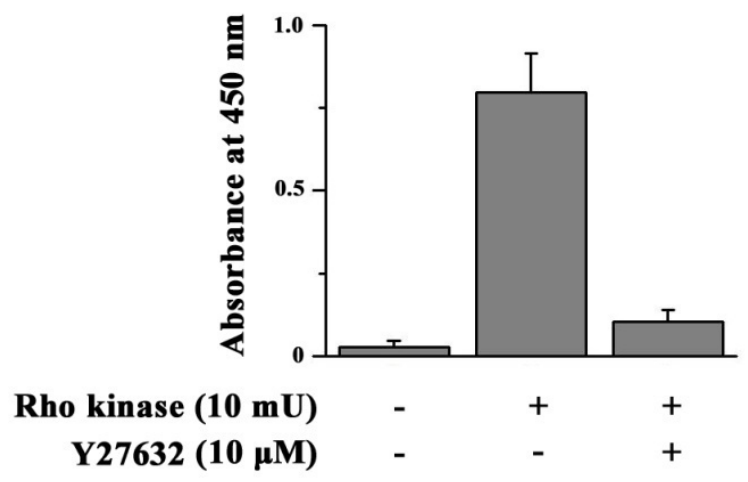

Figure 5-5. The inhibition effect of Y27632 on Rho kinase activity.

The activity of purified Rho kinase II $(10 \mathrm{mU})$ was determined in the presence and absence of $10 \mu \mathrm{M}$ Y27632 using the Rho kinase activity kit. 
stabilization of vascular network structures, we elevated intracellular cAMP by applying a long-acting cAMP derivative, 8-Br-cAMP, to the HMEC tranductants. As shown in Figure 5-6A, elevation in intracellular cAMP by 8 -Br-cAMP $(500 \mu \mathrm{M})$ could completely rescue the unstabilized vascular structures formed by CD151 KD cells. Moreover, the rescuing effect by the cAMP derivative exhibits a dose-dependent manner (Figure 5-6B), suggesting a close functional relationship between cAMP and CD151 in vascular stabilization. These results also provide evidence that cAMP signaling might be the upstream signal of CD151-mediated restraint on RhoA in a manner dependent or independent on Rac1.

cAMP/PKA signaling could regulate vascular stability through Rac1-dependent RhoA inhibition. To further understand the role of Rac1 signaling in CD151-mediated vascular stabilization, we next upregulated Rac 1 activity in endothelial cells by using Rac1 F28L mutant, which fast cycles between GTP- and GDP-loaded forms. ${ }^{201}$ Overexpression of Rac1 F28L mutant in CD151 KD endothelial cells cannot rescue the instability of vascular network structures (Figure 5-6C), indicating Rac1 signaling is not required for CD151-mediated vascular stabilization. Together, our results suggest that CD151 regulates vascular stability through RhoA but not Rac1 and also indicate cAMP signaling directly modulates RhoA activity by PKA-dependent protein phosphorylation.

\subsubsection{CD151-mediated Restraint on RhoA Is Not Dependent on P190Rho ${ }^{\text {GAP }}$ and P115Rho ${ }^{\text {GEF }}$}

As illustrated by Bar-Sagi pathway, ${ }^{180}$ antagonism constantly exists between Rac 1 and RhoA signalings. It was reported that $\mathrm{p} 190 \mathrm{Rho}{ }^{\mathrm{GAP}}$, a RhoA-specific GAP and major regulator of RhoA, coordinates such antagonism by anchoring to adherens junctions. ${ }^{30}$ We next determined if CD151 confines RhoA activity in a p190Rho ${ }^{\text {GAP }}$-dependent manner. Given the fact that active $\mathrm{p} 190 \mathrm{Rho}^{\mathrm{GAP}}$ is phosphorylated at tyrosine residues, we examined the tyrosine phosphorylation levels of p190Rho ${ }^{\mathrm{GAP}}$ in HMEC transfectants and found that the level of tyrosine phosphorylated p190Rho ${ }^{\mathrm{GAP}}$ in CD151 KD cells is comparable to that in MOCK cells (Figure 5-7A). Additionally, we performed a pulldown assay using RhoA Q63L, a constitutively active RhoA mutant which selectively binds the activated, RhoA-specific GAPs, ${ }^{27,202}$ to directly detect the level of active p190Rho ${ }^{\mathrm{GAP}}$. As shown in Figure 5-7B, the amount of p190Rho ${ }^{\text {GAP }}$ coprecipitated with RhoA Q63L remains unchanged in CD151 KD cells compared to MOCK cells. Collectively, p190Rho ${ }^{\mathrm{GAP}}$ activation is not affected by CD151 silencing, and p190Rho ${ }^{\mathrm{GAP}}$ appears not to be crucial for CD151-dependent restraint of RhoA signaling.

Another major upstream regulator for RhoA is $\mathrm{p} 115 \mathrm{Rho}{ }^{\mathrm{GEF}}$, a specific guanine nucleotide exchange factor for RhoA. ${ }^{203}$ We compared MOCK and CD151 KD 
A

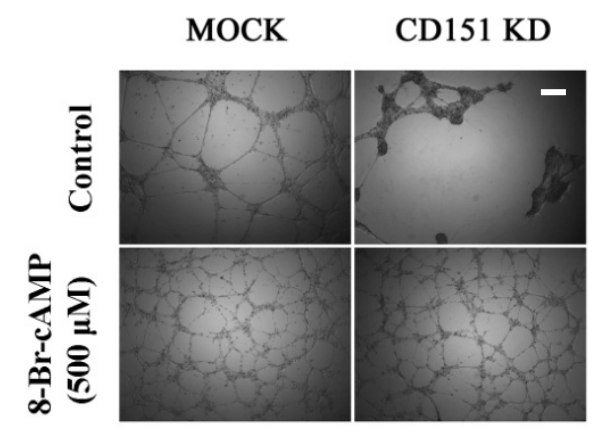

B

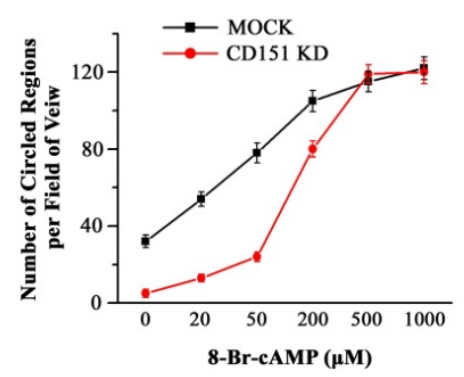

C

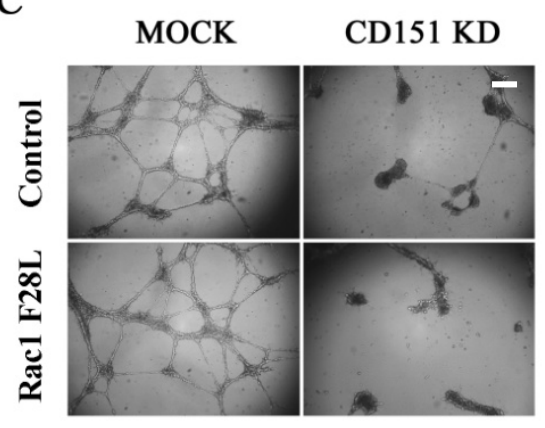

Figure 5-6. Elevation in intracellular cAMP but not active Rac1 rescues the defective vascular network structures caused by loss of CD151.

(A-B) 8-Br-cAMP at various concentrations was applied in HMEC transductants for cable network formation assay. The network structures were photographed at $18 \mathrm{~h}$, and the number of cable cord-enclosed regions was counted from the photographs. Presented are (A) representative images of the cable network structures with treatment of $500 \mu \mathrm{M}$ 8-Br-cAMP, and (B) quantitative data of the network structures in the presence of various concentrations of 8-Br-cAMP. (C) Expressing a fast-cycling Rac1 (F28L) mutant cannot rescue CD151-silencing derived vascular instability. Rac1 F28L with GFP fusion was overexpressed in HMEC transductants by a retroviral construct. Cells were undergone GFP sorting to separate GFP-Rac1 F28L positive and negative cells. Sorted cells were then subjected to the cable network formation assay and photographed at $18 \mathrm{~h}$. Bar $=250$ $\mu \mathrm{m}$. 
A

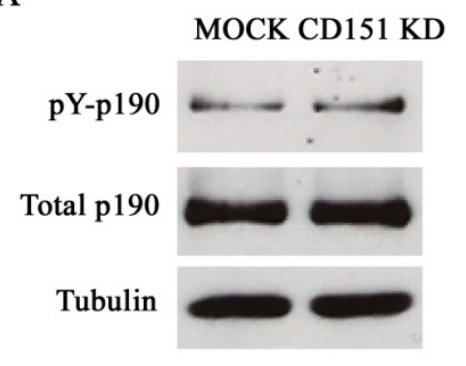

C

MOCK CD151 KD

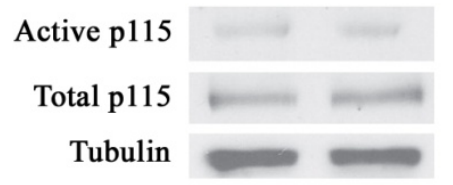

B

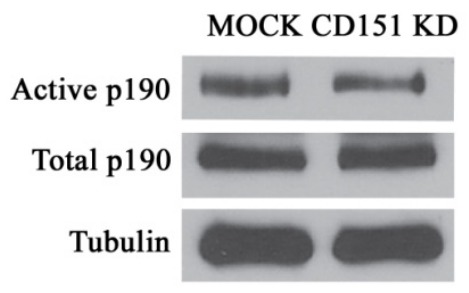

D

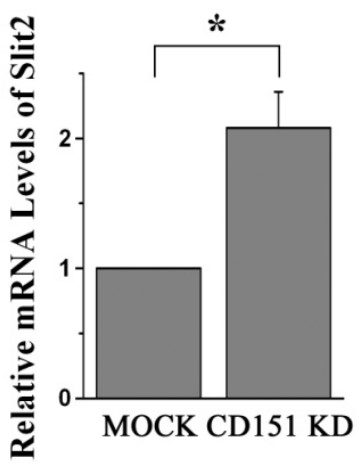

Figure 5-7. Upstream signaling studies.

(A-C) CD151 does not affect p190Rho ${ }^{\mathrm{GAP}}$ and p115Rho ${ }^{\mathrm{GEF}}$ activation. (A) Cellular p190Rho ${ }^{\text {GAP }}$ were IPed with anti-p190Rho ${ }^{\text {GAP }} \mathrm{mAb}$, and the amount of phosphorylated p190Rho ${ }^{\text {GAP }}$ at tyrosine was determined by immunoblotting with anti phospho-tyrosine mAb PY20. After stripping, total precipitated p190 was detected with anti-p190Rho ${ }^{\text {GAP }}$ mAb. (B-C) The active form of p190Rho ${ }^{\mathrm{GAP}}$ (B) or p115Rho ${ }^{\mathrm{GEF}}$ (C) was detected by GST pull down using GST-fused constitutively active RhoA mutant (Q63L) and GST-fused nucleotide-empty RhoA mutant (G17A), respectively. (D) Loss of CD151 upregulates Slit2 expression. The levels of mRNA expression of Slit2 gene were determined by qRT-PCR. * $\mathrm{p}<0.01$. 
endothelial cells for the levels of active p115Rho ${ }^{\mathrm{GEF}}$ using a GST fusion of RhoA G17A mutant, an empty-nucleotide mutant that cannot bind to GTP\&GDP and specifically interacts active p115Rho ${ }^{\text {GEF }}{ }^{202}$ As shown in Figure 5-7C, the amount of the RhoA G17A-bound p115Rho ${ }^{\mathrm{GEF}}$, which represents active pool of p115Rho ${ }^{\mathrm{GEF}}$, in MOCK cells was virtually the same as that in CD151 KD cells, indicating that $\mathrm{p} 115 \mathrm{Rho}{ }^{\mathrm{GEF}}$ is unlikely to be the upstream signal in CD151-induced containment of RhoA signaling.

\subsubsection{CD151 Downregulates Slit2 Expression}

Although CD151 promotes Rac1 and Cdc42 signaling, ${ }^{74,85,112}$ the mechanism remains unknown. Slit2 is the activating ligand of Roundabout proteins, which maintain vascular integrity, and inhibits Rac1 and Cdc42. ${ }^{204,205}$ We assessed the mRNA levels of Slit2 in HMEC transfectants using qRT-PCR and found that the transcript level of Slit2 in CD151 KD cells was increased $\sim 1$ fold compared to that in MOCK cells (Figure 5-7D). Upon CD151 silencing, the upregulated Slit2 gene expression correlates well with the downregulated Rac1 signaling.

\subsection{Summary}

We have demonstrated the role of CD151 in RhoA signaling-mediated cytoskeletal tension and its contribution to vascular stability. CD151 could prevent stress fiber formation by inhibiting RhoA-Rho kinase-MLC signaling, leading to lower cell contractility and tension. Specific inhibitors of RhoA signaling could completely rescue the phenotypic changes in vascular stability caused by loss of CD151. These results suggest that CD151 contributes to vascular stability via its negative regulation in RhoA signaling-mediated stress fiber formation and cellular contractility. In addition, RhoA signaling inhibitor restores endothelial cell-cell adhesiveness, revealing that CD151 may regulate cell-cell adhesion through a RhoA-dependent mechanism.

We next observed downregulation in Rac1 activity and upregulation in gene expression of Slit2 in CD151-silenced HMECs. Moreover, we found elevation in intracellular cAMP could rescue the unstabilized vascular network structures in CD151 endothelial cells. We next excluded the possibility that Rac1 is involved in CD151-mediated stabilization of vasculature, since elevating active Rac1 levels by a fast-cycling Rac1 mutant cannot rescue the deficient vascular network structures. We also provided evidence that the regulation of CD151 on RhoA signaling is p190Rho ${ }^{\text {GAP }}$ - and p115Rho ${ }^{\mathrm{GEF}}$-independent. Together, cAMP signaling but not Rac1, p190Rho ${ }^{\mathrm{GAP}}$ or p115Rho ${ }^{\mathrm{GEF}}$ is the upstream signals of CD151-dependent restraint of RhoA activation. 


\section{CHAPTER 6. DISCUSSION}

\subsection{Introduction}

It has been well established that CD151 is a tumor metastasis promoter. Thus far, it is relatively clear that, through the stable physical association with and functional connection to laminin-binding integrins, CD151 promotes tumor progression by upregulating cell motility, cell adhesion, proteolytic activity of MMPs, and metastasis-related signaling pathways. CD151 was also shown to promote pathological angiogenesis. ${ }^{74}$ How CD151 regulates pathological angiogenesis, however, remains largely unknown.

In the present study, we aimed to define the mechanisms by which CD151 regulates vascular morphogenesis, an essential process involved in vasculogenesis and angiogenesis. In Chapter 2, we characterized a critical role of CD151 in maintaining vascular stability both in vitro and in vivo. In Chapters 3 to 5, we examined the molecular, cellular, and signaling mechanisms by which CD151 stabilizes vascular stability. Our current understanding favors a novel model in which CD151 coordinates multiple biological processes of endothelial cells including cell-matrix adhesion, cell-cell adhesion, and cytoskeletal tension by strengthening the membrane connections of actin cytoskeleton (Figure 6-1). In this chapter, we will discuss this model in detail and define its biological significance.

\subsection{Tetraspanin CD151 Promotes Vascular Stability}

Tetraspanin CD151 is widely expressed in epithelial, smooth muscle, and endothelial cells as well as platelets and megakaryocytes. ${ }^{51,54,68}$ The expression level of CD151 is particularly high in endothelial cells and platelets. Lines of evidence support that CD151 plays important roles in angiogenesis under selective pathologic conditions in vitro and in vivo. ${ }^{73,74,76,89}$ In this study, we found that loss of CD151 expression disrupts vascular stability and results in aberrant vascular permeability. In the absence of CD151, endothelial cells can initiate the formation of capillary network structures on Matrigel but cannot sustain the vascular structures in vitro (Figure 2-4 and supplementary videos). Consistently, ablation of CD151 in mouse causes elevated microvascular permeability under inflammatory condition (Figure 2-5).

Vascular morphogenesis is the process involving the assembly of endothelial cells into capillary structure during vasculogenesis and angiogenesis. ${ }^{1}$ During vascular morphogenesis, numerous factors regulate endothelial cell proliferation, migration, and 
In the absence of CD151

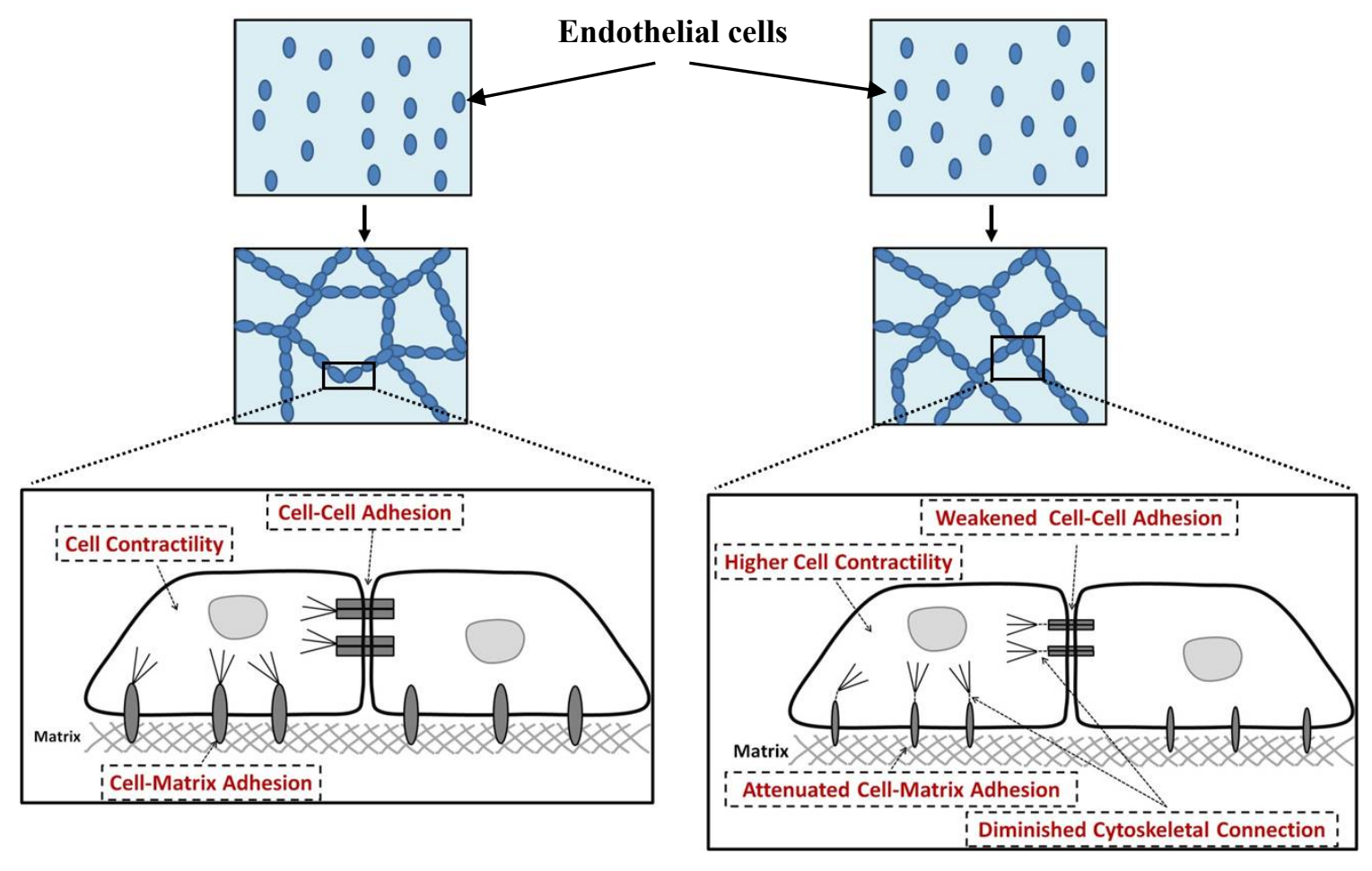

Figure 6-1. A working model for CD151 in stabilizing vascular structures.

(A) After the endothelial network structures are formed, CD151 could balance endothelial cell-matrix adhesion, cell-cell adhesion and cytoskeletal traction and thus stailizes the vasculatures. (B) In the absence of CD151, the endothelial network structures can be formed but not maintained properly. The CD151-negative vasculature exhibits weakened endothelial cell-matrix and cell-cell adhesions and elevated cellular contractility. CD151 might promote cell adhesion through strengthening the connection of actin cytoskeleton to the cell adhesive machinery. 
adhesion, vessel wall assembly, and endothelium structural integrity and stability. ${ }^{1,14,206}$ Therefore, vascular morphogenesis and the stabilization of newly formed vasculatures depend on 1) proper migration and proliferation of endothelial cells and 2) the coordination of cell-cell adhesion, cell-matrix adhesion, and basement membrane assembly. $^{207}$

As demonstrated in live imaging, endothelial cells underwent cell attachment and spreading, active migration and retraction, and dynamic interaction among cells and with surrounding matrix during vascular morphogenesis. Since loss of CD151 disrupts the maintenance of newly formed vascular structure after vascular morphogenesis, CD151 is likely involved in regulating any of cellular processes described above. Our data suggest that loss of CD151 does not markedly affect endothelial cell death and motility but results in impaired cell-matrix adhesiveness, upregulation of RhoA signaling, and aberrant endothelial adherens junctions (diminished cell-cell adhesiveness). It can be envisioned that, CD151 balances cell-matrix adhesiveness, cell-cell adhesion, and cell contraction in endothelium during blood vessel formation and therefore stabilizes the newly formed vasculature. In the absence of CD151, endothelial cells tend to fall off from the underlying matrix due to the impaired cell-matrix interaction, increased intrinsic tension from upregulated RhoA signaling, and diminished cell-cell junctions of the newly formed vasculature. Consequently, CD151-negatively vessels are prone to be unstable and disrupted.

\subsection{CD151 Regulates Multiple Endothelial Cell Events}

CD151 forms stable association with laminin-binding integrins $\alpha 3 \beta 1, \alpha 6 \beta 1, \alpha 6 \beta 4$, and $\alpha 7 \beta 1{ }^{61,71,76}$ It has been well established that CD151, together with the integrins, is involved in a variety of cellular processes including neurite outgrowth and platelet aggregation as well as cell morphogenesis, cell-matrix adhesion strengthening, cell-cell adhesion, and cell migration in epithelial and tumor cells. ${ }^{72,76,79,84,85,91}$ The engineering of endothelial cells without or with diminished CD151 expression has permitted us to investigate the roles of CD151 in endothelial physiology and pathophysiology. Using this experimental system, we not only observed similar mechanism by which CD151 regulates endothelial cell behaviors but also elaborated the general understanding of CD151 biological functions and vascular stability.

\subsubsection{CD151 Does Not Affect Endothelial Cell Death and Cell Motility}

CD151 has not been implicated to modulate cell survival and proliferation or cause apoptosis or necrosis. Indeed, in the cell death assay, we found loss of CD151 does 
not affect endothelial cell viability on Matrigel for as long as 54 hour (Figure 3-1). In addition, earlier observation has shown CD151 ablation has no impact on endothelial cell proliferation. $^{74}$ Thus, we can exclude the possibility that CD151 promotes vascular stability by regulating cell death and proliferation.

Previous studies showed that CD151 enhances tumor invasion and metastasis through regulating integrin-dependent cell migration. ${ }^{72,79,101,102}$ However, we found that CD151 silencing does not significantly alter the hapotactic and chemotactic cell movement in Transwell cell migration assay as well as random cell movement in wound healing assay of microvascular endothelial cells (Figure 3-2). In contrast to a myriad of evidence supporting a regulatory role of CD151 in tumor cell motility, no effect of CD151 on endothelial cell motility suggests that CD151-regulated machinery of cell migration is distinct from cells to cells or CD151-regulated signaling step for cell migration is tissue specific.

\subsubsection{CD151 Optimizes Endothelial Cell-matrix Adhesion}

Cellular interactions with extracellular matrix are essential in angiogenesis. The formation of vasculatures requires the adhesion of endothelial cells to basement membranes. ${ }^{4,207}$ CD151-associated laminin-binding integrins $\alpha 3 \beta 1, \alpha 6 \beta 1$, and $\alpha 6 \beta 4$ play pivotal roles in cell-basement membrane adhesion, ${ }^{140,208,209}$ and CD151 is highly involved in regulating cell-matrix adhesion in epithelial or tumor cells. ${ }^{79,81-83,91}$ We demonstrated in this study that CD151 also strengthens integrin-dependent cell-matrix adhesion in endothelial cells.

First, the reduction or loss of CD151 expression impairs the adhesion of endothelial cells onto laminin 5, the ligand of integrins $\alpha 3 \beta 1$ and $\alpha 6 \beta 4$, in the short-term cell-matrix protein adhesion assay (Figure 3-3A), indicating that CD151 is required for the optimal cell adhesion dependent on integrins $\alpha 3 \beta 1$ and $\alpha 6 \beta 4$. However, CD151 is not needed for the total cell surface expression levels or basal surface distribution of integrin $\alpha 3 \beta 1$ as well as tetraspanin CD9 in endothelial cells (Table 2-1 and Figure 3-4). A recent study demonstrated that CD151 determines the glycosylation of $\alpha 3$ integrin. ${ }^{62} \mathrm{We}$ observed consistant results, as reported in that study, which suggests that loss of CD151 attenuates the glycosylation of $\alpha 3$ integrin subunit in endothelial cells (Figure 3-5). It may be the mechanism by which CD151 strengthens integrin $\alpha 3 \beta 1$-mediated cell-matrix adhesion. In addition, we also observed CD151 strengthens integrins $\alpha 6 \beta 1$ and $\alpha 5 \beta 1$-dependent cell adhesion on fibronectin and laminin 1, respectively (Figure 3-3B). Moreover we found CD151 reinforces the connection of $\beta 1$ integrins to actin cytoskeleton (Figure 3-6). Thus, CD151 likely potentiates the adhesion ability of $\beta 1$ integrins by enhancing the connection of actin cytoskeleton to these integrins. 
Furthermore, CD151 upregulates the expression levels of cell-matrix protein CD44 (Figures 3-3 and 3-8), underlining that CD151 as a global regulator of both integrin-dependent and -independent cell-matrix adhesions in endothelium. Further studies should be directed to identify 1) the protein association of $\beta 1$ integrin subunit with actin network and 2) the mechanism by which CD151 upregulates other cell-matrix adhesion proteins, to depict a complete picture of CD151-mediated strengthening of endothelial cell-matrix adhesion.

The role of CD151 in endothelial cell-matrix adhesion, which is consistent with early observations made from other cell types and from the skin and kidney abnormalities caused by human CD151 mutant, ${ }^{74,79,127}$ helps explain the contributions of CD151 to the endothelial stability of newly formed vessels in angiogenesis. Because endothelial cell-matrix adhesion becomes diminished in the absence of CD151, endothelial cells are likely to be relatively easier to detach from the underlying matrix due to 1) the constant tension from tubulogenesis and branching morphogenesis and 2) shear stress from blood influx, resulting in the disruption of vasculature. Because CD151 strengthens cell adhesion mediated by integrin $\alpha 6 \beta 1$ and potentiates the ligand-binding activity of integrin $\alpha 3 \beta 1$ by stabilizing the activated conformation of this integrin, ${ }^{83,91}$ we activated $\beta 1$ integrins $^{210}$ and rescued the unstable endothelial network structure formed by CD151 KD cells (Figure 3-7), supporting the idea that CD151-dependent vascular stability is at least partially based on its regulation on integrin-mediated cell-matrix adhesion and also suggesting that CD151 is required for the full activation of $\beta 1$ integrins. Despite of being able to fully rescue the defect in endothelial network maintenance caused by CD151 silencing, the activation of $\beta 1$ integrins cannot stabilize the network structure of CD151-silenced group to the level of MOCK, suggesting that CD151 is needed not only for the optimal function of $\beta 1$ integrins but also for other endothelial cellular events required for stabilization of vascular structure in a $\beta 1$ integrin-independent manner.

\subsubsection{CD151 Reinforces Endothelial Cell-cell Adhesion}

Endothelial cell-cell adhesion is critical for blood vessel formation and stability. Molecules responsible for endothelial cell-cell adhesion can be classified into two major groups: $\mathrm{Ca}^{2+}$-dependent cadherins such as VE-cadherin and $\mathrm{Ca}^{2+}$-independent Ig superfamily proteins such as PECAM-1/CD31. VE-cadherin physically and functionally associates with catenins and form adherens junctions. ${ }^{10-12} \mathrm{VE}$-cadherin is essential for the morphogenesis of vessel-like structure in vitro as well as vascular morphogenesis in

vivo. ${ }^{12-14}$ Interestingly, integrin $\alpha 3 \beta 1$ in epithelial cells modulates epithelial cell-cell adhesion and the adhesion-associated cytoskeletal organization possibly through its interaction with E-cadherin junctional complex. Integrin $\alpha 3 \beta 1$ coordinates the signaling from E-cadherin- $\beta$-catenin complex and other pathways such as TGF $\beta 1 /$ Smad pathway 
and is therefore important for establishing and maintaining epithelial adherens junctions. ${ }^{211,212} \mathrm{CD} 151$, in association with integrin $\alpha 3 \beta 1$, was reported to promote epithelial cell-cell adhesion. ${ }^{85,116}$ The role of integrin $\alpha 3 \beta 1-\mathrm{CD} 151$ complex in endothelial cell-cell adhesion remains unknown.

Because diminished endothelial cell-cell adhesion could lead to the instability of newly formed vasculature, CD151 may sustain neovascularization by reinforcing cell-cell interactions. Indeed, we found CD151 promotes $\mathrm{Ca}^{2+}$-dependent endothelial cell-cell adhesiveness through stabilizing endothelial adherens junctions and subsequently maintains vascular stability and barrier function (Figures 4-1 and 4-2).

Next, we defined the signaling mechanism by which CD151 stabilizes endothelial adherens junctions. In epithelium, integrin $\alpha 3 \beta 1-\mathrm{CD} 151$ complex physically associates with E-cadherin junctional complex and stabilizes it. ${ }^{116}$ In endothelium, we demonstrated CD151 is not required for assembly of the junctional complex consisting of VE-cadherin and catenins (Figure 4-4). In contrast, we found that CD151 is likely to be essential for the anchorage of actin filaments from the sub-cortical cytoskeleton meshwork to VE-cadherin junctional complexes at cell-cell contact sites (Figure 4-5), thereby modulating the structural soundness of cell-cell contacts.

In addition, based on the observations from cell aggregation assay, we found that the reduction or loss of $\mathrm{CD} 151$ expression also altered $\mathrm{Ca}^{2+}$-independent cell-cell contacts (Figure 4-1), suggesting that CD151 is also of importance in the functions of other types of cell-cell adhesive molecules such as PECAM-1 and ICAM-2 in vasculature. Previous studies revealed that CD151 regulates the heterotypic cell interactions between leukocyte and endothelial cell in an ICAM-1- and VCAM-1-dependent manner. ${ }^{118}$ CD151 facilitates ICAM-1 and VCAM-1 functions through compartmentalization of these molecules in endothelial cells. ${ }^{118}$ Of note, ICAM-1 and VCAM-1 associates with ERM proteins and form transmembrane complex to anchor cortical cytoskeleton, similar to CD44-mediated cell-matrix adhesion. ${ }^{159-162}$ Thus, we predict that CD151 is involved in modulating PECAM-1-mediated homophilic adhesion between endothelial cells by reorganizing membrane microdomains and reinforcing membrane-cytoskeleton connection.

\subsubsection{CD151 Modulates RhoA Signaling-mediated Endothelial Cytoskeletal Tension}

The activation of RhoA-Rho kinase-MLC signaling axis induces stress fiber formation and cytoskeletal tension (Figure 5-1) ${ }^{17}$ Previous studies have demonstrated that RhoA signaling-mediated cytoskeletal reorganization is crucial for the maintenance of endothelial stability. ${ }^{19-21}$ Excessive RhoA activity disrupts the integrity of blood 
vessels in angiogenesis and selectively induces the regression of vasculatures, whereas the suppression on RhoA signaling promotes angiogenesis and vessel stability. ${ }^{19-21}$ In murine keratinocytes, integrin $\alpha 3$ deletion was found to result in the increase in stress fiber formation. ${ }^{213}$ Moreover, CD151 has also been implicated to regulate RhoA activity in epidermal carcinoma cells. ${ }^{117}$ In endothelial cells, CD151 effects RhoA signaling as it does in epithelial counterpart. Loss of CD151 results in excessive activation of RhoA-Rho kinase-myosin signaling and robust formation of stress fibers (Figures 5-2 and 5-3), leading to the elevated traction of cellular actin cytoskeleton. Our observation from CD151-silencing endothelial cells supports the idea that CD151 stabilizes newly formed blood vessels through suppressing RhoA signaling and stress fiber formation. Hence, due to constantly high intracellular tension, CD151-ablated or -silenced vasculature may undergo regression in an accelerated fashion with minimal effect on initial formation, thereby leading to the disruption of capillary structures. Suppressions of RhoA signaling axis by specific inhibitors C3 transferase, Y27632 and Blebbistatin in CD151-ablated or -silenced endothelial cells completely block the collapse of endothelial cable network and maintain the stability of capillary structures (Figure 5-4). Of note, the inhibitors might have completely blocked the activities of RhoA signaling pathway, i.e., not only the excessive portions from the loss of CD151 but also the endogenous activities responsible for its physiological roles. Such inhibitions helped the endothelial cells to better sustain the capillary structures, further supporting that RhoA signaling promotes the regression of vasculatures. Together, these findings indicate that CD151 promotes vascular stability in a manner dependent on RhoA-mediated cytoskeletal tension.

Several observations have implicated that tetraspanins are important regulators of small GTPase RhoA. ${ }^{117,214,215}$ However, the signaling cascade linking tetraspanins to RhoA is still unclear. As shown in the diagram in Figure 5-1, many signal molecules, such as p115 Rho ${ }^{\mathrm{GEF}}$, p190Rho ${ }^{\mathrm{GAP}}$ and the cAMP-dependent protein kinase A (PKA), function as direct upstream regulator of RhoA. We analyzed these signal pathways to reveal the upstream signal of CD151-dependent RhoA regulation.

P115Rho ${ }^{\text {GEF }}$ is a RhoA-specific GEF, which catalyzes the exchange of GDP for GTP in RhoA and thus specifically activates RhoA signaling pathway. ${ }^{203}$ CD151 silencing does not change the level of active p115Rho ${ }^{\mathrm{GEF}}$ (Figure 5-7C), indicating that CD151 is not required for p115Rho ${ }^{\mathrm{GEF}}$ activation. Thus, the upregulation of RhoA signaling upon CD151 silencing is independent of $\mathrm{p} 115 \mathrm{Rho}{ }^{\mathrm{GEF}}$. Because $\mathrm{p} 115 \mathrm{Rho}{ }^{\mathrm{GEF}}$ is typically activated by GPCRs when GPCRs bind to their ligands such as hormones and chemokines, ${ }^{187,188}$ the reduction in or loss of CD151 expression appears not to fall into this category. Thus, our observation of the unaltered p115Rho ${ }^{\mathrm{GEF}}$ activation upon CD151 silencing is consistent with such a criterion.

Among the regulators specific to RhoA, p190Rho ${ }^{\mathrm{GAP}}$ is the most extensively 
studied one. P190Rho ${ }^{\text {GAP }}$ terminates GTPase activity of RhoA by hydrolysis of GTP to GDP. ${ }^{30}$ RhoA can be suppressed by $\mathrm{p} 190 \mathrm{Rho}^{\mathrm{GAP}}$ in an integrin-dependent manner.

Integrin-initiated signaling during cell attachment and spreading activates $\mathrm{p} 190 \mathrm{Rho}{ }^{\mathrm{GAP}}$ by inducing the translocation of $\mathrm{p} 190 \mathrm{Rho}{ }^{\mathrm{GAP}}$ to the plasma membrane and phosphorylation of p190Rho ${ }^{\text {GAP }}$ (Figure 5-1). ${ }^{184-186}$ A similar signaling mechanism may contribute to CD151-dependent restraint of RhoA because of the functional overlapping between CD151 and integrins. However, we found that the diminution or loss of CD151 expression does not change the p190Rho ${ }^{\text {GAP }}$ activation, i.e., neither level of its tyrosine phosphorylation nor level of the p190Rho ${ }^{\text {GAP }}$ proteins that bind to the constitutively active RhoA (Figure 5-7A and B), indicating that CD151 is not required for p190Rho ${ }^{\text {GAP }}$ activation. This finding also reveals that CD151-mediated restraint of RhoA or CD151 silencing-induced upregulation of RhoA is independent of p190Rho ${ }^{\mathrm{GAP}}$.

CAMP/PKA signaling-mediated phosphorylation provides another type of regulatory mechanism for RhoA. ${ }^{189,190}$ Since we observed that elevation of intracellular cAMP could rescue the unstabilized vascular structures formed by CD151-silenced endothelial cells in a dose-dependent manner (Figure 5-6A and B), we therefore provide evidence that cAMP signaling is intimately involved in CD151-dependent vascular stabilization. Together with the finding that CD151 restrains RhoA activation and the fact that the protective effect of cAMP on endothelial barrier properties is at least partially dependent on Rho ${ }^{196-200}$, we conclude that cAMP/PKA signaling is very likely the direct upstream signal of CD151-dependent RhoA restraint. Our findings favor a novel signaling transduction model (Figure 6-2) in which CD151/integrin complexes-dependent signaling trigger production of cAMP and the subsequent activation of PKA, which in turn phosphorylates RhoA and thus suppresses RhoA activity. As described previously, de-activation of RhoA by PKA might be dependent on the diminshed RhoA association with Rho kinase and enhanced interaction of RhoA with RhoGDI and translocation of membrane-localized RhoA to the cytosol. ${ }^{189,190,199}$ Thus, the phosphorylated RhoA cannot activate Rho kinase and MLC and induce stress fiber formation. In the absence of CD151, insufficient cAMP production leads to deficiency in RhoA phosphorylation and excessive activation of RhoA signaling.

\subsubsection{CD151 Might Be an Important Regulator of Cortical Cytoskeletal Organization}

Our results imply that CD151 restrains RhoA activation and stress fiber formation and sustains Rac1 activity (Figure 5-3D). Together with previous observations from tumor cells and CD151-ablated endothelial cells, ${ }^{12,117,214,215}$ these findings suggest an important role of CD151 in Rho small GTPase-mediated cytoskeletal reorganization during cell-matrix and cell-cell adhesions. In addition, the diminution or loss of CD151 expression significantly weakens the associations of $\beta 1$ integrins and VE-cadherin with 


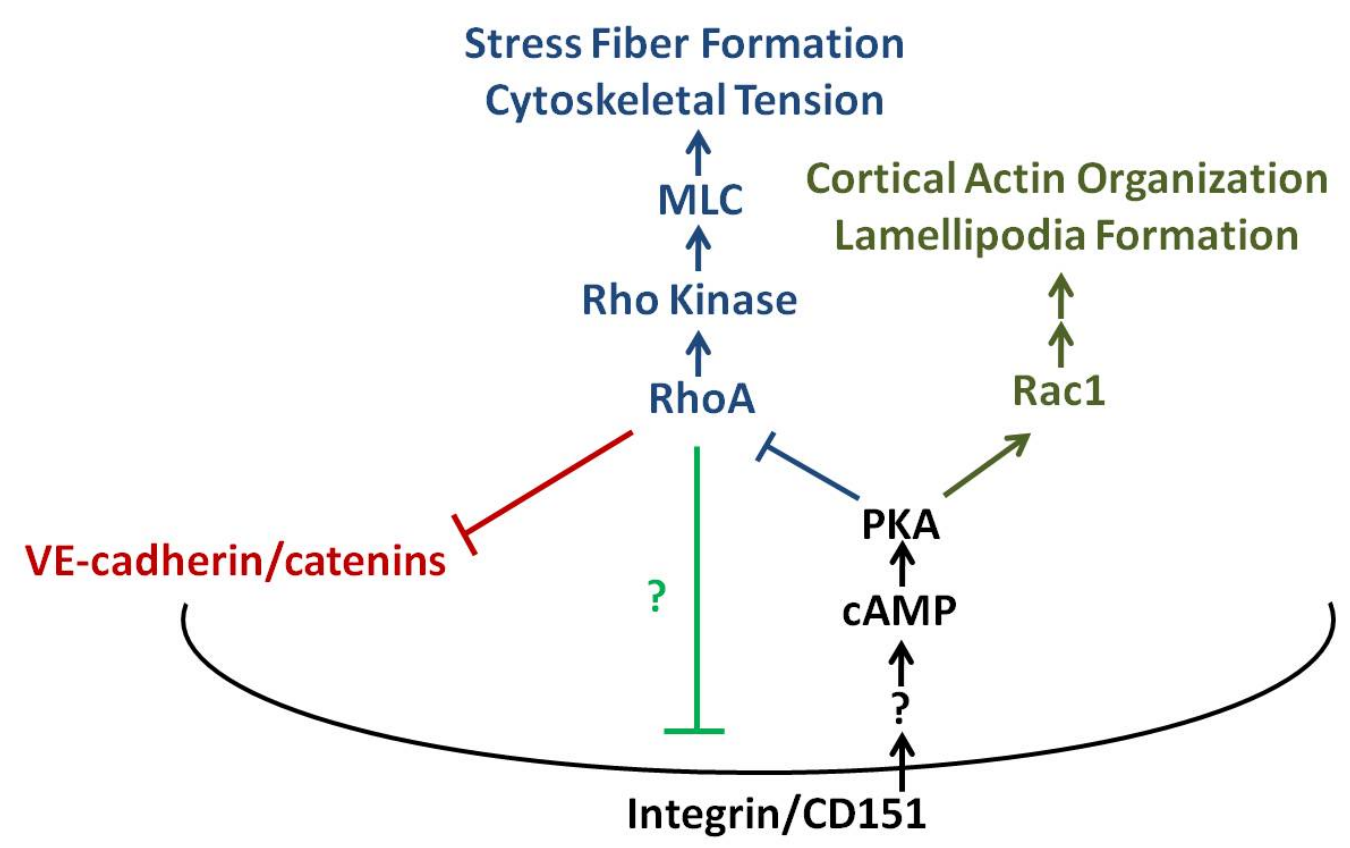

Figure 6-2. The signaling of integrin/CD151 complexes in endothelial cells.

CD151 binds to integrins and activates cAMP/PKA signaling, by which CD151 restrains RhoA signaling and promotes Rac1 signaling. Therefore CD151 suppresses RhoA-mediated cytoskeletal orgranizations and lowers cytoskeletal tension. CD151-dependent restraint on RhoA and activation of Rac1, on the other hand, stabilize cortical actin meshwork and reinforce the connection of the membrane adhesive proteins, such as VE-cadherin and integrins, to actin filments, causing globally strengthening of endothelial cell adhesiveness and subsequently leading to vascular stability. 
detergent-insoluble cytoskeleton (Figures 3-6 and 4-5), strongly suggesting that CD151 strengthens the connection of the cell adhesion proteins in the plasma membrane to cytoskeletal network, possibly via RhoA (Figure 6-2).

Moreover, we found that CD151 silencing reduced the expression of CD44, a cell-matrix adhesion molecule. Studies from elsewhere indicate that CD151 promotes endothelial VCAM-1- and ICAM-1-mediated heterotypic cell-cell adhesion. ${ }^{118}$ Given the facts that CD44, VCAM-1, and ICAM-1 bind to actin cytoskeleton through ERM proteins, it is predictable that CD151 indirectly modulates ERM-mediated anchorage of cytoskeleton to the plasma membrane. Collectively, CD151 likely regulates the reorganization of cortical actin cytoskeleton, especially the membrane linkage of cytoskeleton. A logical explanation for the global effect of CD151 on cortical cytoskeleton organization is that CD151 expression may directly or indirectly (through RhoA?) alter the biophysical properties of the plasma membrane. As CD151 was shown to compartmentalize membrane proteins in the plasma membrane, ${ }^{44,63,79,216}$ CD151-dependent interaction between membrane and cytoskeleton may thus affect the order, curvature, and fluidity of the plasma membrane.

\subsection{CD151 Coordinates Cell Adhesions and Actin Cytoskeletal Tension}

\subsubsection{CD151 Strengthens Cell-cell and Cell-matrix Adhesions and Regulates the Crosstalk between Them}

Cellular interactions among cells and with the surrounding matrix are fundamentally important for tissue morphogenesis. The organisms assemble cells and extracellular matrix into three-dimensional structures to form tissues. ${ }^{179}$ Therefore it is believed that the establishments of adhesion structures could be central events for tissue morphogenesis. ${ }^{179}$ Also, the maintenance of the established cell adhesion structures is obviously critical for tissue structural stability. From the structure biology point of view, desmosomes and hemidesmosomes share very similar architecture. ${ }^{217}$ Moreover, the adhesion complexes at cell-cell and cell-matrix contacts consist of the same components such as vinculin, $\alpha$-actinin and talin, which are localized at the cytoplasmic face of both cell-matrix and cell-cell adhesions and directly linked to actin cytoskeleton. ${ }^{218}$ On the other hand, cells have also developed a regulatory mechanism through which a single molecule can be used to control both cell-cell adhesion and cell-matrix adhesion. A well-known example is $\beta$-catenin. This protein has two distinct roles: one as a constitutient of adherens junctions and another as a signaling transducer in Wnt signaling

pathway which crosstalks with integrin signaling. ${ }^{219,220}$ It can be envisioned that cell-cell adhesion and cell-matrix adhesion are coordinated events during and after development. 
CD151 distributes at both basal and lateral cell surfaces where it colocalizes with cadherins and integrins, the two major adhesion molecules engaging cell-cell and cell-matrix adhesions, respectively. In CD151-silenced endothelial cells, the connections of actin cytoskeleton to both cell-cell and cell-matrix adhesion complexes become less rigorous (Figures 3-6 and 4-5), suggesting that CD151 very likely coordinates cell-cell and cell-matrix interactions through the reorganization of actin cytoskeleton. CD151 mediates the crosstalk of these two processes probably by regulating the functions of common components in cell-cell and cell-matrix adhesions such as the cytoskeletal "crosslinkers" vinculin, $\alpha$-actinin, and talin in a RhoA-dependent manner. It remains to be determined in future studies.

\subsubsection{CD151 Regulates Cell-cell Adhesion and Cytoskeletal Tension}

Cell-cell adhesion and cell contraction are closely interconnected. ${ }^{29}$ Cadherin and Rho family GTPase signalings are also mutually regulated. ${ }^{25-28}$ For example, RhoA-dependent cytoskeletal tension promotes the disassembly of cell-cell adhesion via myosin II-mediated actin reorganization. ${ }^{29}$ Expression of constitutively activated RhoA disrupts adherens junctions, where the inhibitors of RhoA signaling reinforce cell-cell adhesions. ${ }^{26}$ On the other hands, the adherens junctional complex, which consists of cadherin, $\beta$-catenin, and $120^{\mathrm{ctn}}$, inhibits RhoA signaling. ${ }^{30}$ Our observations and previous findings in cancer cells underscore the interplay between cell-cell adhesion and cell contraction because the Rho kinase inhibitor Y27632 could restore the dislocalized AJs, caused by loss of CD151, at cell-cell contacts ${ }^{117}$ and result in fully recovered $\mathrm{Ca}^{2+}$-dependent cell-cell adhesiveness in CD151-silenced cells (Figure 4-1). Hence, CD151 is likely involved in the signaling crosstalk between cell contraction/retraction and cell-cell adhesion via upregulating RhoA activity (Figure 6-2).

\subsubsection{CD151 Balances RhoA and Rac1 Signalings}

Recent publications reveal apparently conflicting roles of CD151 in regulating Rac1 activity in different experimental systems. The observations from CD151 knockout mouse and overexpression cells indicated that CD151 promotes Rac1 activity, ${ }^{74,85}$ whereas CD151 silencing in carcinoma cells does not alter Rac1 signaling. ${ }^{117}$ We have shown that CD151 is needed for maintaining Rac1 activity in endothelial cells. The diminution or loss of CD151 expression results in attenuated Rac1 activity and elevated RhoA activity (Figure 5-3), which are in consistence with the antagonism between Rac1 and RhoA signalings as described in the Bar-Sagi pathway. ${ }^{180}$ Moreover, cAMP signaling stabilizes vascular structure by either inducing Rac1 activity, inhibiting RhoA activation, 
or both, ${ }^{196-200}$ and we indeed observed that elevated level of intracellular cAMP rescues the phenotypes of CD151-silenced endothelial cells (Figure 5-6A and B). These results provide evidence that cAMP signaling might be the upstream signal of CD151-mediated restraint on RhoA in a manner dependent or independent on Rac1. However, based on the observation from Rac1 fast-cycling mutant (Figure 5-6C), we are able to exclude the possibility that Rac1 may play a role in CD151-dependent restraint of RhoA activity and maintenance of endothelial stability. Since elevated level of active Rac1 in CD151-silenced endothelial cells cannot rescue the deficiency in endothelial stability caused by the loss of CD151, CD151-dependent maintenances of endothelial stability in vascular morphogenesis and Rac1 activity are likely to be independent with each other. Most likely, as summarized in Figure 6-2, cAMP/PKA signaling serves as upstream signal which differentially modulates both RhoA and Rac1 activity.

Moreover, as described previously, increased Rac1 signaling likely results in the activation and translocation of $\mathrm{p} 190 \mathrm{Rho}{ }^{\mathrm{GAP}} \cdot{ }^{30}$ It is also believed that, to strengthen adherens junctions, Rac 1 facilitates the assembly of cadherin-catenin complex probably by promoting the association of activated p190Rho ${ }^{\text {GAP }}$ with the adherens junctions, leading to the local inactivation of RhoA (Figure 5-1). In contrast, RhoA suppresses cadherin-mediated adhesive activity by restraining the establishment of sub-cortical actin cytoskeleton without affecting cadherin-catenin complex (Figure 5-1). ${ }^{25,30}$ Since diminution or loss of CD151 expression alters neither protein-protein interactions within the junctional complex consisting of VE-cadherin and $\beta$-catenin nor activation of p190Rho ${ }^{\mathrm{GAP}}$ (Figures 4-4 and 5-7A and B), CD151 reinforces adherens junctions more likely through confining RhoA but not through activating Rac1. Together, the observations support the idea that CD151 balances Rac1 and RhoA signalings in a cAMP/PKA signaling-dependent manner (Figure 6-2).

We next determined whether CD151 regulates Slit2 gene expression. Slit2 is the ligand of Roundabouts (Robos). Upon binding to Robo, Slit2 inhibits the activities of small GTPases Rac1 and Cdc42 and plays important roles in axon guidance and smooth muscle cell migration. ${ }^{205,221}$ We found that diminution or loss of CD151 expression results in elevated Slit2 expression level (Figure 5-7D), which exhibits consistency with the downregulated Rac1 activity in CD151-silenced cells, suggesting that CD151 sustains Rac1 activity, at least partially, through Slit2-Robo signaling. On the other hand, in the absence of CD151, a high level of Slit2 expression may suppress the nuclear accumulation of $\beta$-catenin, as demonstrated in lung cancer cells, ${ }^{222}$ which in turn inhibits the gene expression driven by $\beta$-catenin, e.g., CD44 expression, and eventually renders cells less adhesive and invasive. In endothelial cells, Slit2 activates an endothelial-specific Robo, Robo4. It has been reported that Slit2-Robo4 signaling promotes vascular stability by inhibiting Rac1-mediated cell migration. ${ }^{204}$ It appears to be conflicting with our observation that, in the absence of CD151, the upregulation of Slit2 
expression is accompanied by diminished endothelial stability. This discrepancy is explainable. In the presence of CD151, Slit2-Robo4 signaling-mediated inhibition of Rac1 promotes vascular stability. However, when CD151 expression is diminished or lost, the disruptive effects from deficient cell-cell and cell-matrix adhesions and high cytoskeletal tension likely override the beneficial effect from Slit2-mediated inhibition of cell migration, eventually leading to vascular instability.

\subsection{Summary and Remarks}

In the present study, we revealed the mechanisms by which CD151 regulates vascular morphogenesis and stability. Our results support the notion that CD151 is needed for proper endothelial stability of blood vessels. CD151 reinforces endothelial cell-matrix and cell-cell adhesions and alleviates cell intrinsic traction or contractility. Our findings also revealed a novel paradigm in which CD151 functions as signaling switch to balance the activities of Rac1 and RhoA probably through regulating the global connection of the plasma membrane to actin cytoskeleton, leading to the endothelail stability.

Our present study has highlighted the cellular and molecular mechanisms by which CD151 regulates vascular stability. However, several critical questions, some of which we have mentioned above, emerge from the study. For example, how do CD151 regulate cAMP signaling? Why CD151 has a globe regulatory effect on cortical cytoskeleton anchorage. Do CD151 determine the biophysical state of plasma membrane? To answer these questions, future studies may focus on the regulations of CD151 in 1) cAMP activation and/or compartmentalization, 2) functions/associations of common components in cell-cell and cell-matrix adhesions such as the cytoskeletal "crosslinkers" vinculin, $\alpha$-actinin, and talin, and 3 ) the order, curvature, and fluidity of the plasma membrane as well as coordination of the physical properties of plasma membrane and cortical cytoskeletal organization. Moreover, genetically modified animals with dominant negative mutants of CD151 will be necessary for interpretation of loss-of-CD151-derived phenotypic changes and for better understanding of roles of CD151 in vivo as functional compensation by other tetraspanins might exist in CD151-deleted and-silenced models. 


\section{LIST OF REFERENCES}

1. Davis, G.E., Bayless, K.J. and Mavila, A. Molecular basis of endothelial cell morphogenesis in three-dimensional extracellular matrices. Anat Rec 268, 252-275 (2002).

2. Bischoff, J. Cell adhesion and angiogenesis. J Clin Invest 99, 373-376 (1997).

3. Stromblad, S. and Cheresh, D.A. Cell adhesion and angiogenesis. Trends Cell Biol 6, 462-468 (1996).

4. Hynes, R.O. Cell-matrix adhesion in vascular development. $J$ Thromb Haemost 5 Suppl 1, 32-40 (2007).

5. Hynes, R.O. Integrins: versatility, modulation, and signaling in cell adhesion. Cell 69, 11-25 (1992).

6. Van der Flier, A. and Sonnenberg, A. Function and interactions of integrins. Cell Tissue Res 305, 285-298 (2001).

7. Goh, K.L., Yang, J.T. and Hynes, R.O. Mesodermal defects and cranial neural crest apoptosis in alpha5 integrin-null embryos. Development 124, 4309-4319 (1997).

8. Griffioen, A.W., Coenen, M.J., Damen, C.A., Hellwig, S.M., van Weering, D.H., Vooys, W., Blijham, G.H. and Groenewegen, G. CD44 is involved in tumor angiogenesis; an activation antigen on human endothelial cells. Blood $\mathbf{9 0}$, 1150-1159 (1997).

9. Cao, G., Savani, R.C., Fehrenbach, M., Lyons, C., Zhang, L., Coukos, G. and Delisser, H.M. Involvement of endothelial CD44 during in vivo angiogenesis. Am J Pathol 169, 325-336 (2006).

10. Venkiteswaran, K., Xiao, K., Summers, S., Calkins, C.C., Vincent, P.A., Pumiglia, K. and Kowalczyk, A.P. Regulation of endothelial barrier function and growth by VE-cadherin, plakoglobin, and beta-catenin. Am J Physiol Cell Physiol 283, C811-821 (2002).

11. Bazzoni, G. and Dejana, E. Endothelial cell-to-cell junctions: molecular organization and role in vascular homeostasis. Physiol Rev 84, 869-901 (2004). 
12. Vestweber, D. Molecular mechanisms that control endothelial cell contacts. $J$ Pathol 190, 281-291 (2000).

13. Corada, M., Liao, F., Lindgren, M., Lampugnani, M.G., Breviario, F., Frank, R., Muller, W.A., Hicklin, D.J., Bohlen, P. and Dejana, E. Monoclonal antibodies directed to different regions of vascular endothelial cadherin extracellular domain affect adhesion and clustering of the protein and modulate endothelial permeability. Blood 97, 1679-1684 (2001).

14. Dejana, E., Spagnuolo, R. and Bazzoni, G. Interendothelial junctions and their role in the control of angiogenesis, vascular permeability and leukocyte transmigration. Thromb Haemost 86, 308-315 (2001).

15. Liao, F., Li, Y., O'Connor, W., Zanetta, L., Bassi, R., Santiago, A., Overholser, J., Hooper, A., Mignatti, P., Dejana, E., Hicklin, D.J. and Bohlen, P. Monoclonal antibody to vascular endothelial-cadherin is a potent inhibitor of angiogenesis, tumor growth, and metastasis. Cancer Res 60, 6805-6810 (2000).

16. Huang, M.T., Mason, J.C., Birdsey, G.M., Amsellem, V., Gerwin, N., Haskard, D.O., Ridley, A.J. and Randi, A.M. Endothelial intercellular adhesion molecule (ICAM)-2 regulates angiogenesis. Blood 106, 1636-1643 (2005).

17. Swetman, C.A., Leverrier, Y., Garg, R., Gan, C.H., Ridley, A.J., Katz, D.R. and Chain, B.M. Extension, retraction and contraction in the formation of a dendritic cell dendrite: distinct roles for Rho GTPases. Eur J Immunol 32, 2074-2083 (2002).

18. Bayless, K.J. and Davis, G.E. The Cdc42 and Rac1 GTPases are required for capillary lumen formation in three-dimensional extracellular matrices. $J$ Cell Sci 115, 1123-1136 (2002).

19. Im, E. and Kazlauskas, A. Src family kinases promote vessel stability by antagonizing the Rho/ROCK pathway. J Biol Chem 282, 29122-29129 (2007).

20. Bayless, K.J. and Davis, G.E. Microtubule depolymerization rapidly collapses capillary tube networks in vitro and angiogenic vessels in vivo through the small GTPase Rho. J Biol Chem 279, 11686-11695 (2004).

21. Mavria, G., Vercoulen, Y., Yeo, M., Paterson, H., Karasarides, M., Marais, R., Bird, D. and Marshall, C.J. ERK-MAPK signaling opposes Rho-kinase to promote endothelial cell survival and sprouting during angiogenesis. Cancer Cell 9, 33-44 (2006). 
22. Sawada, N., Salomone, S., Kim, H.H., Kwiatkowski, D.J. and Liao, J.K. Regulation of endothelial nitric oxide synthase and postnatal angiogenesis by Rac1. Circ Res 103, 360-368 (2008).

23. Dormond, O., Foletti, A., Paroz, C. and Ruegg, C. NSAIDs inhibit alpha V beta 3 integrin-mediated and $\mathrm{Cdc} 42 /$ Rac-dependent endothelial-cell spreading, migration and angiogenesis. Nat Med 7, 1041-1047 (2001).

24. Koh, W., Mahan, R.D. and Davis, G.E. Cdc42- and Rac1-mediated endothelial lumen formation requires Pak2, Pak4 and Par3, and PKC-dependent signaling. $J$ Cell Sci 121, 989-1001 (2008).

25. Fukata, M. and Kaibuchi, K. Rho-family GTPases in cadherin-mediated cell-cell adhesion. Nat Rev Mol Cell Biol 2, 887-897 (2001).

26. Fang, W.B., Ireton, R.C., Zhuang, G., Takahashi, T., Reynolds, A. and Chen, J. Overexpression of EPHA2 receptor destabilizes adherens junctions via a RhoA-dependent mechanism. J Cell Sci 121, 358-368 (2008).

27. Noren, N.K., Niessen, C.M., Gumbiner, B.M. and Burridge, K. Cadherin engagement regulates Rho family GTPases. J Biol Chem 276, 33305-33308 (2001).

28. Ren, X.D., Kiosses, W.B. and Schwartz, M.A. Regulation of the small GTP-binding protein Rho by cell adhesion and the cytoskeleton. Embo J 18, 578-585 (1999).

29. Schwartz, M.A. and Horwitz, A.R. Integrating adhesion, protrusion, and contraction during cell migration. Cell 125, 1223-1225 (2006).

30. Wildenberg, G.A., Dohn, M.R., Carnahan, R.H., Davis, M.A., Lobdell, N.A., Settleman, J. and Reynolds, A.B. p120-catenin and p190RhoGAP regulate cell-cell adhesion by coordinating antagonism between Rac and Rho. Cell 127, 1027-1039 (2006).

31. Hemler, M.E. Tetraspanin functions and associated microdomains. Nat Rev Mol Cell Biol 6, 801-811 (2005).

32. Wright, M.D. and Tomlinson, M.G. The ins and outs of the transmembrane 4 superfamily. Immunol Today 15, 588-594 (1994). 
33. Maecker, H.T., Todd, S.C. and Levy, S. The tetraspanin superfamily: molecular facilitators. Faseb J 11, 428-442 (1997).

34. Horejsi, V. and Vlcek, C. Novel structurally distinct family of leucocyte surface glycoproteins including CD9, CD37, CD53 and CD63. FEBS Lett 288, 1-4 (1991).

35. Boucheix, C. and Rubinstein, E. Tetraspanins. Cell Mol Life Sci 58, 1189-1205 (2001).

36. Stipp, C.S., Kolesnikova, T.V. and Hemler, M.E. EWI-2 is a major CD9 and CD81 partner and member of a novel Ig protein subfamily. J Biol Chem 276, 40545-40554 (2001).

37. Rubinstein, E., Le Naour, F., Lagaudriere-Gesbert, C., Billard, M., Conjeaud, H. and Boucheix, C. CD9, CD63, CD81, and CD82 are components of a surface tetraspan network connected to HLA-DR and VLA integrins. Eur J Immunol 26, 2657-2665 (1996).

38. Levy, S. and Shoham, T. Protein-protein interactions in the tetraspanin web. Physiology (Bethesda) 20, 218-224 (2005).

39. Zoller, M. Tetraspanins: push and pull in suppressing and promoting metastasis. Nat Rev Cancer 9, 40-55 (2009).

40. Lazo, P.A. Functional implications of tetraspanin proteins in cancer biology. Cancer Sci 98, 1666-1677 (2007).

41. Goschnick, M.W. and Jackson, D.E. Tetraspanins-structural and signalling scaffolds that regulate platelet function. Mini Rev Med Chem 7, 1248-1254 (2007).

42. Wright, M.D., Moseley, G.W. and van Spriel, A.B. Tetraspanin microdomains in immune cell signalling and malignant disease. Tissue Antigens 64, 533-542 (2004).

43. Martin, F., Roth, D.M., Jans, D.A., Pouton, C.W., Partridge, L.J., Monk, P.N. and Moseley, G.W. Tetraspanins in viral infections: a fundamental role in viral biology? J Virol 79, 10839-10851 (2005). 
44. Liu, L., He, B., Liu, W.M., Zhou, D., Cox, J.V. and Zhang, X.A. Tetraspanin CD151 promotes cell migration by regulating integrin trafficking. J Biol Chem 282, 31631-31642 (2007).

45. Berditchevski, F. and Odintsova, E. Tetraspanins as regulators of protein trafficking. Traffic 8, 89-96 (2007).

46. Sugiura, T. and Berditchevski, F. Function of alpha3beta1-tetraspanin protein complexes in tumor cell invasion. Evidence for the role of the complexes in production of matrix metalloproteinase 2 (MMP-2). J Cell Biol 146, 1375-1389 (1999).

47. Saito, Y., Tachibana, I., Takeda, Y., Yamane, H., He, P., Suzuki, M., Minami, S., Kijima, T., Yoshida, M., Kumagai, T., Osaki, T. and Kawase, I. Absence of CD9 enhances adhesion-dependent morphologic differentiation, survival, and matrix metalloproteinase-2 production in small cell lung cancer cells. Cancer Res $\mathbf{6 6}$, 9557-9565 (2006).

48. Arduise, C., Abache, T., Li, L., Billard, M., Chabanon, A., Ludwig, A., Mauduit, P., Boucheix, C., Rubinstein, E. and Le Naour, F. Tetraspanins regulate ADAM10-mediated cleavage of TNF-alpha and epidermal growth factor. $J$ Immunol 181, 7002-7013 (2008).

49. Yanez-Mo, M., Barreiro, O., Gonzalo, P., Batista, A., Megias, D., Genis, L., Sachs, N., Sala-Valdes, M., Alonso, M.A., Montoya, M.C., Sonnenberg, A., Arroyo, A.G. and Sanchez-Madrid, F. MT1-MMP collagenolytic activity is regulated through association with tetraspanin CD151 in primary endothelial cells. Blood 112, 3217-3226 (2008).

50. Zhang, F., Kotha, J., Jennings, L.K. and Zhang, X.A. Tetraspanins and vascular functions. Cardiovasc Res 83, 7-15 (2009).

51. Fitter, S., Tetaz, T.J., Berndt, M.C. and Ashman, L.K. Molecular cloning of cDNA encoding a novel platelet-endothelial cell tetra-span antigen, PETA-3. Blood 86, 1348-1355 (1995).

52. Whittock, N.V. and McLean, W.H. Genomic organization, amplification, fine mapping, and intragenic polymorphisms of the human hemidesmosomal tetraspanin CD151 gene. Biochem Biophys Res Commun 281, 425-430 (2001). 
53. Hasegawa, H., Watanabe, H., Nomura, T., Utsunomiya, Y., Yanagisawa, K. and Fujita, S. Molecular cloning and expression of mouse homologue of SFA-1/PETA-3 (CD151), a member of the transmembrane 4 superfamily. Biochim Biophys Acta 1353, 125-130 (1997).

54. Fitter, S., Seldin, M.F. and Ashman, L.K. Characterisation of the mouse homologue of CD151 (PETA-3/SFA-1); genomic structure, chromosomal localisation and identification of 2 novel splice forms. Biochim Biophys Acta 1398, 75-85 (1998).

55. Hasegawa, H., Kishimoto, K., Yanagisawa, K., Terasaki, H., Shimadzu, M. and Fujita, S. Assignment of SFA-1 (PETA-3), a member of the transmembrane 4 superfamily, to human chromosome $11 \mathrm{p} 15.5$ by fluorescence in situ hybridization. Genomics 40, 193-196 (1997).

56. Wang, J., Liu, X., Ni, P., Gu, Z. and Fan, Q. SP1 is required for basal activation and chromatin accessibility of CD151 promoter in liver cancer cells. Biochem Biophys Res Commun 393, 291-296 (2010).

57. Olweus, J., Lund-Johansen, F. and Horejsi, V. CD53, a protein with four membrane-spanning domains, mediates signal transduction in human monocytes and B cells. J Immunol 151, 707-716 (1993).

58. Sterk, L.M., Geuijen, C.A., Oomen, L.C., Calafat, J., Janssen, H. and Sonnenberg, A. The tetraspan molecule CD151, a novel constituent of hemidesmosomes, associates with the integrin alpha6beta4 and may regulate the spatial organization of hemidesmosomes. J Cell Biol 149, 969-982 (2000).

59. Yauch, R.L., Berditchevski, F., Harler, M.B., Reichner, J. and Hemler, M.E. Highly stoichiometric, stable, and specific association of integrin alpha3beta1 with CD151 provides a major link to phosphatidylinositol 4-kinase, and may regulate cell migration. Mol Biol Cell 9, 2751-2765 (1998).

60. Yauch, R.L., Kazarov, A.R., Desai, B., Lee, R.T. and Hemler, M.E. Direct extracellular contact between integrin alpha(3)beta(1) and TM4SF protein CD151. J Biol Chem 275, 9230-9238 (2000).

61. Berditchevski, F., Gilbert, E., Griffiths, M.R., Fitter, S., Ashman, L. and Jenner, S.J. Analysis of the CD151-alpha3beta1 integrin and CD151-tetraspanin interactions by mutagenesis. J Biol Chem 276, $41165-41174$ (2001). 
62. Baldwin, G., Novitskaya, V., Sadej, R., Pochec, E., Litynska, A., Hartmann, C., Williams, J., Ashman, L., Eble, J.A. and Berditchevski, F. Tetraspanin CD151 regulates glycosylation of (alpha)3(beta)1 integrin. J Biol Chem 283, 35445-35454 (2008).

63. Berditchevski, F., Odintsova, E., Sawada, S. and Gilbert, E. Expression of the palmitoylation-deficient CD151 weakens the association of alpha 3 beta 1 integrin with the tetraspanin-enriched microdomains and affects integrin-dependent signaling. J Biol Chem 277, 36991-37000 (2002).

64. Yang, X., Claas, C., Kraeft, S.K., Chen, L.B., Wang, Z., Kreidberg, J.A. and Hemler, M.E. Palmitoylation of tetraspanin proteins: modulation of CD151 lateral interactions, subcellular distribution, and integrin-dependent cell morphology. Mol Biol Cell 13, 767-781 (2002).

65. Sharma, C., Yang, X.H. and Hemler, M.E. DHHC2 affects palmitoylation, stability, and functions of tetraspanins CD9 and CD151. Mol Biol Cell 19, 3415-3425 (2008).

66. Charrin, S., Manie, S., Oualid, M., Billard, M., Boucheix, C. and Rubinstein, E. Differential stability of tetraspanin/tetraspanin interactions: role of palmitoylation. FEBS Lett 516, 139-144 (2002).

67. Sincock, P.M., Mayrhofer, G. and Ashman, L.K. Localization of the transmembrane 4 superfamily (TM4SF) member PETA-3 (CD151) in normal human tissues: comparison with CD9, CD63, and alpha5beta1 integrin. $J$ Histochem Cytochem 45, 515-525 (1997).

68. Geary, S.M., Cambareri, A.C., Sincock, P.M., Fitter, S. and Ashman, L.K. Differential tissue expression of epitopes of the tetraspanin CD151 recognised by monoclonal antibodies. Tissue Antigens 58, 141-153 (2001).

69. Serru, V., Le Naour, F., Billard, M., Azorsa, D.O., Lanza, F., Boucheix, C. and Rubinstein, E. Selective tetraspan-integrin complexes (CD81/alpha4beta1, CD151/alpha3beta1, CD151/alpha6beta1) under conditions disrupting tetraspan interactions. Biochem J 340 (Pt 1), 103-111 (1999).

70. Fitter, S., Sincock, P.M., Jolliffe, C.N. and Ashman, L.K. Transmembrane 4 superfamily protein CD151 (PETA-3) associates with beta 1 and alpha IIb beta 3 integrins in haemopoietic cell lines and modulates cell-cell adhesion. Biochem $J$ 338 (Pt 1), 61-70 (1999). 
71. Sterk, L.M., Geuijen, C.A., van den Berg, J.G., Claessen, N., Weening, J.J. and Sonnenberg, A. Association of the tetraspanin CD151 with the laminin-binding integrins alpha3beta1, alpha6beta1, alpha6beta4 and alpha7beta1 in cells in culture and in vivo. J Cell Sci 115, 1161-1173 (2002).

72. Gesierich, S., Paret, C., Hildebrand, D., Weitz, J., Zgraggen, K., Schmitz-Winnenthal, F.H., Horejsi, V., Yoshie, O., Herlyn, D., Ashman, L.K. and Zoller, M. Colocalization of the tetraspanins, CO-029 and CD151, with integrins in human pancreatic adenocarcinoma: impact on cell motility. Clin Cancer Res 11, 2840-2852 (2005).

73. Sincock, P.M., Fitter, S., Parton, R.G., Berndt, M.C., Gamble, J.R. and Ashman, L.K. PETA-3/CD151, a member of the transmembrane 4 superfamily, is localised to the plasma membrane and endocytic system of endothelial cells, associates with multiple integrins and modulates cell function. J Cell Sci 112 (Pt 6), 833-844 (1999).

74. Takeda, Y., Kazarov, A.R., Butterfield, C.E., Hopkins, B.D., Benjamin, L.E., Kaipainen, A. and Hemler, M.E. Deletion of tetraspanin Cd151 results in decreased pathologic angiogenesis in vivo and in vitro. Blood 109, 1524-1532 (2007).

75. Yamada, M., Tamura, Y., Sanzen, N., Sato-Nishiuchi, R., Hasegawa, H., Ashman, L.K., Rubinstein, E., Yanez-Mo, M., Sanchez-Madrid, F. and Sekiguchi, K. Probing the interaction of tetraspanin CD151 with integrin alpha 3 beta 1 using a panel of monoclonal antibodies with distinct reactivities toward the CD151-integrin alpha 3 beta 1 complex. Biochem J 415, 417-427 (2008).

76. Zhang, X.A., Kazarov, A.R., Yang, X., Bontrager, A.L., Stipp, C.S. and Hemler, M.E. Function of the tetraspanin CD151-alpha6betal integrin complex during cellular morphogenesis. Mol Biol Cell 13, 1-11 (2002).

77. Charrin, S., Manie, S., Billard, M., Ashman, L., Gerlier, D., Boucheix, C. and Rubinstein, E. Multiple levels of interactions within the tetraspanin web. Biochem Biophys Res Commun 304, 107-112 (2003).

78. Kovalenko, O.V., Yang, X., Kolesnikova, T.V. and Hemler, M.E. Evidence for specific tetraspanin homodimers: inhibition of palmitoylation makes cysteine residues available for cross-linking. Biochem J 377, 407-417 (2004). 
79. Winterwood, N.E., Varzavand, A., Meland, M.N., Ashman, L.K. and Stipp, C.S. A critical role for tetraspanin CD151 in alpha3beta1 and alpha6beta4 integrin-dependent tumor cell functions on laminin-5. Mol Biol Cell 17, 2707-2721 (2006).

80. Yanez-Mo, M., Alfranca, A., Cabanas, C., Marazuela, M., Tejedor, R., Ursa, M.A., Ashman, L.K., de Landazuri, M.O. and Sanchez-Madrid, F. Regulation of endothelial cell motility by complexes of tetraspan molecules CD81/TAPA-1 and CD151/PETA-3 with alpha3 beta1 integrin localized at endothelial lateral junctions. J Cell Biol 141, 791-804 (1998).

81. Berditchevski, F. and Odintsova, E. Characterization of integrin-tetraspanin adhesion complexes: role of tetraspanins in integrin signaling. J Cell Biol 146, 477-492 (1999).

82. Yamada, M., Sumida, Y., Fujibayashi, A., Fukaguchi, K., Sanzen, N., Nishiuchi, R. and Sekiguchi, K. The tetraspanin CD151 regulates cell morphology and intracellular signaling on laminin-511. Febs $J \mathbf{2 7 5}, 3335-3351$ (2008).

83. Nishiuchi, R., Sanzen, N., Nada, S., Sumida, Y., Wada, Y., Okada, M., Takagi, J., Hasegawa, H. and Sekiguchi, K. Potentiation of the ligand-binding activity of integrin alpha3beta1 via association with tetraspanin CD151. Proc Natl Acad Sci U S A 102, 1939-1944 (2005).

84. Stipp, C.S. and Hemler, M.E. Transmembrane-4-superfamily proteins CD151 and CD81 associate with alpha 3 beta 1 integrin, and selectively contribute to alpha 3 beta 1-dependent neurite outgrowth. J Cell Sci 113 (Pt 11), 1871-1882 (2000).

85. Shigeta, M., Sanzen, N., Ozawa, M., Gu, J., Hasegawa, H. and Sekiguchi, K. $\mathrm{CD} 151$ regulates epithelial cell-cell adhesion through PKC- and Cdc42-dependent actin cytoskeletal reorganization. J Cell Biol 163, 165-176 (2003).

86. Zhang, X.A., Bontrager, A.L. and Hemler, M.E. Transmembrane-4 superfamily proteins associate with activated protein kinase $\mathrm{C}(\mathrm{PKC})$ and link $\mathrm{PKC}$ to specific beta(1) integrins. J Biol Chem 276, 25005-25013 (2001).

87. Klosek, S.K., Nakashiro, K., Hara, S., Shintani, S., Hasegawa, H. and Hamakawa, H. CD151 forms a functional complex with c-Met in human salivary gland cancer cells. Biochem Biophys Res Commun 336, 408-416 (2005). 
88. Zheng, Z.Z. and Liu, Z.X. Activation of the phosphatidylinositol 3-kinase/protein kinase Akt pathway mediates CD151-induced endothelial cell proliferation and cell migration. Int J Biochem Cell Biol 39, 340-348 (2007).

89. Zheng, Z. and Liu, Z. CD151 gene delivery activates PI3K/Akt pathway and promotes neovascularization after myocardial infarction in rats. Mol Med 12, 214-220 (2006).

90. Klosek, S.K., Nakashiro, K., Hara, S., Goda, H., Hasegawa, H. and Hamakawa, H. CD151 regulates HGF-stimulated morphogenesis of human breast cancer cells. Biochem Biophys Res Commun 379, 1097-1100 (2009).

91. Lammerding, J., Kazarov, A.R., Huang, H., Lee, R.T. and Hemler, M.E. Tetraspanin CD151 regulates alpha6betal integrin adhesion strengthening. Proc Natl Acad Sci U S A 100, 7616-7621 (2003).

92. Previtali, S.C., Dina, G., Nodari, A., Fasolini, M., Wrabetz, L., Mayer, U., Feltri, M.L. and Quattrini, A. Schwann cells synthesize alpha7beta 1 integrin which is dispensable for peripheral nerve development and myelination. Mol Cell Neurosci 23, 210-218 (2003).

93. Chernousov, M.A., Kaufman, S.J., Stahl, R.C., Rothblum, K. and Carey, D.J. Alpha7beta1 integrin is a receptor for laminin-2 on Schwann cells. Glia 55, 1134-1144 (2007).

94. Burkin, D.J. and Kaufman, S.J. The alpha7beta1 integrin in muscle development and disease. Cell Tissue Res 296, 183-190 (1999).

95. Kaariainen, M., Nissinen, L., Kaufman, S., Sonnenberg, A., Jarvinen, M., Heino, J. and Kalimo, H. Expression of alpha7beta1 integrin splicing variants during skeletal muscle regeneration. Am J Pathol 161, 1023-1031 (2002).

96. Ke, A.W., Shi, G.M., Zhou, J., Wu, F.Z., Ding, Z.B., Hu, M.Y., Xu, Y., Song, Z.J., Wang, Z.J., Wu, J.C., Bai, D.S., Li, J.C., Liu, K.D. and Fan, J. Role of overexpression of CD151 and/or c-Met in predicting prognosis of hepatocellular carcinoma. Hepatology 49, 491-503 (2009).

97. Tokuhara, T., Hasegawa, H., Hattori, N., Ishida, H., Taki, T., Tachibana, S., Sasaki, S. and Miyake, M. Clinical significance of CD151 gene expression in non-small cell lung cancer. Clin Cancer Res 7, 4109-4114 (2001). 
98. Sadej, R., Romanska, H., Kavanagh, D., Baldwin, G., Takahashi, T., Kalia, N. and Berditchevski, F. Tetraspanin CD151 regulates transforming growth factor beta signaling: implication in tumor metastasis. Cancer Res 70, 6059-6070 (2010).

99. Ang, J., Lijovic, M., Ashman, L.K., Kan, K. and Frauman, A.G. CD151 protein expression predicts the clinical outcome of low-grade primary prostate cancer better than histologic grading: a new prognostic indicator? Cancer Epidemiol Biomarkers Prev 13, 1717-1721 (2004).

100. Woegerbauer, M., Thurnher, D., Houben, R., Pammer, J., Kloimstein, P., Heiduschka, G., Petzelbauer, P. and Erovic, B.M. Expression of the tetraspanins CD9, CD37, CD63, and CD151 in Merkel cell carcinoma: strong evidence for a posttranscriptional fine-tuning of CD9 gene expression. Mod Pathol 23, 751-762 (2010).

101. Testa, J.E., Brooks, P.C., Lin, J.M. and Quigley, J.P. Eukaryotic expression cloning with an antimetastatic monoclonal antibody identifies a tetraspanin (PETA-3/CD151) as an effector of human tumor cell migration and metastasis. Cancer Res 59, 3812-3820 (1999).

102. Zijlstra, A., Lewis, J., Degryse, B., Stuhlmann, H. and Quigley, J.P. The inhibition of tumor cell intravasation and subsequent metastasis via regulation of in vivo tumor cell motility by the tetraspanin CD151. Cancer Cell 13, 221-234 (2008).

103. Chien, C.W., Lin, S.C., Lai, Y.Y., Lin, B.W., Lin, S.C., Lee, J.C. and Tsai, S.J. Regulation of CD151 by hypoxia controls cell adhesion and metastasis in colorectal cancer. Clin Cancer Res 14, 8043-8051 (2008).

104. Kohno, M., Hasegawa, H., Miyake, M., Yamamoto, T. and Fujita, S. CD151 enhances cell motility and metastasis of cancer cells in the presence of focal adhesion kinase. Int J Cancer 97, 336-343 (2002).

105. Engers, R. and Gabbert, H.E. Mechanisms of tumor metastasis: cell biological aspects and clinical implications. J Cancer Res Clin Oncol 126, 682-692 (2000).

106. Keller, E.T. Overview of metastasis and metastases. J Musculoskelet Neuronal Interact 2, 567-569 (2002). 
107. Onn, A. and Herbst, R.S. Angiogenesis, metastasis, and lung cancer. An overview. Methods Mol Med 74, 329-348 (2003).

108. Garcia-Lopez, M.A., Barreiro, O., Garcia-Diez, A., Sanchez-Madrid, F. and Penas, P.F. Role of tetraspanins CD9 and CD151 in primary melanocyte motility. J Invest Dermatol 125, 1001-1009 (2005).

109. Novitskaya, V., Romanska, H., Dawoud, M., Jones, J.L. and Berditchevski, F. Tetraspanin CD151 regulates growth of mammary epithelial cells in three-dimensional extracellular matrix: implication for mammary ductal carcinoma in situ. Cancer Res 70, 4698-4708 (2010).

110. Hong, I.K., Jin, Y.J., Byun, H.J., Jeoung, D.I., Kim, Y.M. and Lee, H. Homophilic interactions of tetraspanin CD151 up-regulate motility and matrix metalloproteinase-9 expression of human melanoma cells through adhesion-dependent c-Jun activation signaling pathways. J Biol Chem 281, 24279-24292 (2006).

111. Nobes, C.D. and Hall, A. Rho, rac, and cdc42 GTPases regulate the assembly of multimolecular focal complexes associated with actin stress fibers, lamellipodia, and filopodia. Cell 81, 53-62 (1995).

112. Yang, X.H., Richardson, A.L., Torres-Arzayus, M.I., Zhou, P., Sharma, C., Kazarov, A.R., Andzelm, M.M., Strominger, J.L., Brown, M. and Hemler, M.E. CD151 accelerates breast cancer by regulating alpha 6 integrin function, signaling, and molecular organization. Cancer Res 68, 3204-3213 (2008).

113. Shiomi, T., Inoki, I., Kataoka, F., Ohtsuka, T., Hashimoto, G., Nemori, R. and Okada, Y. Pericellular activation of proMMP-7 (promatrilysin-1) through interaction with CD151. Lab Invest 85, 1489-1506 (2005).

114. Fujita, Y., Shiomi, T., Yanagimoto, S., Matsumoto, H., Toyama, Y. and Okada, Y. Tetraspanin CD151 is expressed in osteoarthritic cartilage and is involved in pericellular activation of pro-matrix metalloproteinase 7 in osteoarthritic chondrocytes. Arthritis Rheum 54, 3233-3243 (2006).

115. Hasegawa, M., Furuya, M., Kasuya, Y., Nishiyama, M., Sugiura, T., Nikaido, T., Momota, Y., Ichinose, M. and Kimura, S. CD151 dynamics in carcinoma-stroma interaction: integrin expression, adhesion strength and proteolytic activity. Lab Invest 87, 882-892 (2007). 
116. Chattopadhyay, N., Wang, Z., Ashman, L.K., Brady-Kalnay, S.M. and Kreidberg, J.A. alpha3beta1 integrin-CD151, a component of the cadherin-catenin complex, regulates PTPmu expression and cell-cell adhesion. J Cell Biol 163, 1351-1362 (2003).

117. Johnson, J.L., Winterwood, N., DeMali, K.A. and Stipp, C.S. Tetraspanin CD151 regulates RhoA activation and the dynamic stability of carcinoma cell-cell contacts. J Cell Sci 122, 2263-2273 (2009).

118. Barreiro, O., Yanez-Mo, M., Sala-Valdes, M., Gutierrez-Lopez, M.D., Ovalle, S., Higginbottom, A., Monk, P.N., Cabanas, C. and Sanchez-Madrid, F. Endothelial tetraspanin microdomains regulate leukocyte firm adhesion during extravasation. Blood 105, 2852-2861 (2005).

119. Vasioukhin, V., Bauer, C., Yin, M. and Fuchs, E. Directed actin polymerization is the driving force for epithelial cell-cell adhesion. Cell 100, 209-219 (2000).

120. Niessen, C.M. Tight junctions/adherens junctions: basic structure and function. $J$ Invest Dermatol 127, 2525-2532 (2007).

121. Zavadil, J. and Bottinger, E.P. TGF-beta and epithelial-to-mesenchymal transitions. Oncogene 24, 5764-5774 (2005).

122. John, A. and Tuszynski, G. The role of matrix metalloproteinases in tumor angiogenesis and tumor metastasis. Pathol Oncol Res 7, 14-23 (2001).

123. Deryugina, E.I. and Quigley, J.P. Matrix metalloproteinases and tumor metastasis. Cancer Metastasis Rev 25, 9-34 (2006).

124. Schmierer, B. and Hill, C.S. TGFbeta-SMAD signal transduction: molecular specificity and functional flexibility. Nat Rev Mol Cell Biol 8, 970-982 (2007).

125. Reynolds, L.E., Conti, F.J., Silva, R., Robinson, S.D., Iyer, V., Rudling, R., Cross, B., Nye, E., Hart, I.R., Dipersio, C.M. and Hodivala-Dilke, K.M. alpha3beta1 integrin-controlled Smad7 regulates reepithelialization during wound healing in mice. J Clin Invest 118, 965-974 (2008).

126. Kim, K.K., Wei, Y., Szekeres, C., Kugler, M.C., Wolters, P.J., Hill, M.L., Frank, J.A., Brumwell, A.N., Wheeler, S.E., Kreidberg, J.A. and Chapman, H.A. Epithelial cell alpha3beta1 integrin links beta-catenin and Smad signaling to promote myofibroblast formation and pulmonary fibrosis. J Clin Invest 119, 213-224 (2009). 
127. Karamatic Crew, V., Burton, N., Kagan, A., Green, C.A., Levene, C., Flinter, F., Brady, R.L., Daniels, G. and Anstee, D.J. CD151, the first member of the tetraspanin (TM4) superfamily detected on erythrocytes, is essential for the correct assembly of human basement membranes in kidney and skin. Blood 104, 2217-2223 (2004).

128. Cowin, A.J., Adams, D., Geary, S.M., Wright, M.D., Jones, J.C. and Ashman, L.K. Wound healing is defective in mice lacking tetraspanin CD151. J Invest Dermatol 126, 680-689 (2006).

129. Lau, L.M., Wee, J.L., Wright, M.D., Moseley, G.W., Hogarth, P.M., Ashman, L.K. and Jackson, D.E. The tetraspanin superfamily member CD151 regulates outside-in integrin alphaIIbbeta3 signaling and platelet function. Blood 104, 2368-2375 (2004).

130. Wright, M.D., Geary, S.M., Fitter, S., Moseley, G.W., Lau, L.M., Sheng, K.C., Apostolopoulos, V., Stanley, E.G., Jackson, D.E. and Ashman, L.K. Characterization of mice lacking the tetraspanin superfamily member CD151. Mol Cell Biol 24, 5978-5988 (2004).

131. Orlowski, E., Chand, R., Yip, J., Wong, C., Goschnick, M.W., Wright, M.D., Ashman, L.K. and Jackson, D.E. A platelet tetraspanin superfamily member, CD151, is required for regulation of thrombus growth and stability in vivo. $J$ Thromb Haemost 7, 2074-2084 (2009).

132. Hasegawa, H., Nomura, T., Kishimoto, K., Yanagisawa, K. and Fujita, S. SFA-1/PETA-3 (CD151), a member of the transmembrane 4 superfamily, associates preferentially with alpha 5 beta 1 integrin and regulates adhesion of human $\mathrm{T}$ cell leukemia virus type 1 -infected $\mathrm{T}$ cells to fibronectin. J Immunol 161, 3087-3095 (1998).

133. Hasegawa, H., Utsunomiya, Y., Kishimoto, K., Yanagisawa, K. and Fujita, S. SFA-1, a novel cellular gene induced by human T-cell leukemia virus type 1 , is a member of the transmembrane 4 superfamily. $J$ Virol 70, 3258-3263 (1996).

134. Sheng, K.C., van Spriel, A.B., Gartlan, K.H., Sofi, M., Apostolopoulos, V., Ashman, L. and Wright, M.D. Tetraspanins CD37 and CD151 differentially regulate Ag presentation and T-cell co-stimulation by DC. Eur J Immunol 39, 50-55 (2009). 
135. Ho, S.H., Martin, F., Higginbottom, A., Partridge, L.J., Parthasarathy, V., Moseley, G.W., Lopez, P., Cheng-Mayer, C. and Monk, P.N. Recombinant extracellular domains of tetraspanin proteins are potent inhibitors of the infection of macrophages by human immunodeficiency virus type 1.J Virol 80, 6487-6496 (2006).

136. Sachs, N., Kreft, M., van den Bergh Weerman, M.A., Beynon, A.J., Peters, T.A., Weening, J.J. and Sonnenberg, A. Kidney failure in mice lacking the tetraspanin CD151. J Cell Biol 175, 33-39 (2006).

137. Geary, S.M., Cowin, A.J., Copeland, B., Baleato, R.M., Miyazaki, K. and Ashman, L.K. The role of the tetraspanin CD151 in primary keratinocyte and fibroblast functions: implications for wound healing. Exp Cell Res 314, 2165-2175 (2008).

138. Baleato, R.M., Guthrie, P.L., Gubler, M.C., Ashman, L.K. and Roselli, S. Deletion of CD151 results in a strain-dependent glomerular disease due to severe alterations of the glomerular basement membrane. Am J Pathol 173, 927-937 (2008).

139. Kreidberg, J.A., Donovan, M.J., Goldstein, S.L., Rennke, H., Shepherd, K., Jones, R.C. and Jaenisch, R. Alpha 3 beta 1 integrin has a crucial role in kidney and lung organogenesis. Development 122, 3537-3547 (1996).

140. Kreidberg, J.A. Functions of alpha3beta1 integrin. Curr Opin Cell Biol 12, 548-553 (2000).

141. Mitchell, K., Szekeres, C., Milano, V., Svenson, K.B., Nilsen-Hamilton, M., Kreidberg, J.A. and DiPersio, C.M. Alpha3betal integrin in epidermis promotes wound angiogenesis and keratinocyte-to-endothelial-cell crosstalk through the induction of MRP3. J Cell Sci 122, 1778-1787 (2009).

142. Fukushi, J., Makagiansar, I.T. and Stallcup, W.B. NG2 proteoglycan promotes endothelial cell motility and angiogenesis via engagement of galectin-3 and alpha3beta1 integrin. Mol Biol Cell 15, 3580-3590 (2004).

143. Chandrasekaran, L., He, C.Z., Al-Barazi, H., Krutzsch, H.C., Iruela-Arispe, M.L. and Roberts, D.D. Cell contact-dependent activation of alpha3beta1 integrin modulates endothelial cell responses to thrombospondin-1. Mol Biol Cell 11, 2885-2900 (2000). 
144. Elbashir, S.M., Harborth, J., Lendeckel, W., Yalcin, A., Weber, K. and Tuschl, T. Duplexes of 21-nucleotide RNAs mediate RNA interference in cultured mammalian cells. Nature 411, 494-498 (2001).

145. Brummelkamp, T.R., Bernards, R. and Agami, R. A system for stable expression of short interfering RNAs in mammalian cells. Science 296, 550-553 (2002).

146. Brummelkamp, T.R., Bernards, R. and Agami, R. Stable suppression of tumorigenicity by virus-mediated RNA interference. Cancer Cell 2, 243-247 (2002).

147. Barton, G.M. and Medzhitov, R. Retroviral delivery of small interfering RNA into primary cells. Proc Natl Acad Sci U S A 99, 14943-14945 (2002).

148. Bari, R., Zhang, Y.H., Zhang, F., Wang, N.X., Stipp, C.S., Zheng, J.J. and Zhang, X.A. Transmembrane interactions are needed for KAI1/CD82-mediated suppression of cancer invasion and metastasis. Am J Pathol 174, 647-660 (2009).

149. Thurston, G., Rudge, J.S., Ioffe, E., Zhou, H., Ross, L., Croll, S.D., Glazer, N., Holash, J., McDonald, D.M. and Yancopoulos, G.D. Angiopoietin-1 protects the adult vasculature against plasma leakage. Nat Med 6, 460-463 (2000).

150. Eichten, A., Hyun, W.C. and Coussens, L.M. Distinctive features of angiogenesis and lymphangiogenesis determine their functionality during de novo tumor development. Cancer Res 67, 5211-5220 (2007).

151. Arroyo, A.G., Sanchez-Mateos, P., Campanero, M.R., Martin-Padura, I., Dejana, E. and Sanchez-Madrid, F. Regulation of the VLA integrin-ligand interactions through the beta 1 subunit. J Cell Biol 117, 659-670 (1992).

152. Lim, Y.C., Garcia-Cardena, G., Allport, J.R., Zervoglos, M., Connolly, A.J., Gimbrone, M.A., Jr. and Luscinskas, F.W. Heterogeneity of endothelial cells from different organ sites in T-cell subset recruitment. Am J Pathol 162, 1591-1601 (2003).

153. Voyta, J.C., Via, D.P., Butterfield, C.E. and Zetter, B.R. Identification and isolation of endothelial cells based on their increased uptake of acetylated-low density lipoprotein. J Cell Biol 99, 2034-2040 (1984).

154. Walsh, D.A. Pathophysiological mechanisms of angiogenesis. Adv Clin Chem 44, 187-221 (2007). 
155. Burridge, K., Nuckolls, G., Otey, C., Pavalko, F., Simon, K. and Turner, C. Actin-membrane interaction in focal adhesions. Cell Differ Dev 32, 337-342 (1990).

156. Calderwood, D.A., Zent, R., Grant, R., Rees, D.J., Hynes, R.O. and Ginsberg, M.H. The Talin head domain binds to integrin beta subunit cytoplasmic tails and regulates integrin activation. $J$ Biol Chem 274, 28071-28074 (1999).

157. Arold, S.T., Hoellerer, M.K. and Noble, M.E. The structural basis of localization and signaling by the focal adhesion targeting domain. Structure 10, 319-327 (2002).

158. Petit, V. and Thiery, J.P. Focal adhesions: structure and dynamics. Biol Cell 92, 477-494 (2000).

159. Legg, J.W. and Isacke, C.M. Identification and functional analysis of the ezrin-binding site in the hyaluronan receptor, CD44. Curr Biol 8, 705-708 (1998).

160. Yonemura, S., Hirao, M., Doi, Y., Takahashi, N., Kondo, T., Tsukita, S. and Tsukita, S. Ezrin/radixin/moesin (ERM) proteins bind to a positively charged amino acid cluster in the juxta-membrane cytoplasmic domain of CD44, CD43, and ICAM-2. J Cell Biol 140, 885-895 (1998).

161. Martin, T.A., Harrison, G., Mansel, R.E. and Jiang, W.G. The role of the CD44/ezrin complex in cancer metastasis. Crit Rev Oncol Hematol 46, 165-186 (2003).

162. Barreiro, O., Yanez-Mo, M., Serrador, J.M., Montoya, M.C., Vicente-Manzanares, M., Tejedor, R., Furthmayr, H. and Sanchez-Madrid, F. Dynamic interaction of VCAM-1 and ICAM-1 with moesin and ezrin in a novel endothelial docking structure for adherent leukocytes. J Cell Biol 157, 1233-1245 (2002).

163. Zhang, X.A., He, B., Zhou, B. and Liu, L. Requirement of the p130CAS-Crk coupling for metastasis suppressor KAI1/CD82-mediated inhibition of cell migration. J Biol Chem 278, 27319-27328 (2003).

164. Rodriguez, L.G., Wu, X. and Guan, J.L. Wound-healing assay. Methods Mol Biol 294, 23-29 (2005). 
165. He, B., Liu, L., Cook, G.A., Grgurevich, S., Jennings, L.K. and Zhang, X.A. Tetraspanin CD82 attenuates cellular morphogenesis through down-regulating integrin alpha6-mediated cell adhesion. J Biol Chem 280, 3346-3354 (2005).

166. Kelly, D.J., Azeloglu, E.U., Kochupura, P.V., Sharma, G.S. and Gaudette, G.R. Accuracy and reproducibility of a subpixel extended phase correlation method to determine micron level displacements in the heart. Med Eng Phys 29, 154-162 (2007).

167. Sabass, B., Gardel, M.L., Waterman, C.M. and Schwarz, U.S. High resolution traction force microscopy based on experimental and computational advances. Biophys J 94, 207-220 (2008).

168. Butler, J.P., Tolic-Norrelykke, I.M., Fabry, B. and Fredberg, J.J. Traction fields, moments, and strain energy that cells exert on their surroundings. Am J Physiol Cell Physiol 282, C595-605 (2002).

169. Heid, C.A., StEvans, J., Livak, K.J. and Williams, P.M. Real time quantitative PCR. Genome Res 6, 986-994 (1996).

170. Beningo, K.A. and Wang, Y.L. Flexible substrata for the detection of cellular traction forces. Trends Cell Biol 12, 79-84 (2002).

171. Roy, P., Rajfur, Z., Pomorski, P. and Jacobson, K. Microscope-based techniques to study cell adhesion and migration. Nat Cell Biol 4, E91-96 (2002).

172. Goodison, S., Urquidi, V. and Tarin, D. CD44 cell adhesion molecules. Mol Pathol 52, 189-196 (1999).

173. Tsukita, S. and Yonemura, S. Cortical actin organization: lessons from ERM (ezrin/radixin/moesin) proteins. J Biol Chem 274, 34507-34510 (1999).

174. Montero-Balaguer, M., Swirsding, K., Orsenigo, F., Cotelli, F., Mione, M. and Dejana, E. Stable vascular connections and remodeling require full expression of VE-cadherin in zebrafish embryos. PLoS One 4, e5772 (2009).

175. Vestweber, D., Winderlich, M., Cagna, G. and Nottebaum, A.F. Cell adhesion dynamics at endothelial junctions: VE-cadherin as a major player. Trends Cell Biol 19, 8-15 (2009). 
176. Albelda, S.M., Muller, W.A., Buck, C.A. and Newman, P.J. Molecular and cellular properties of PECAM-1 (endoCAM/CD31): a novel vascular cell-cell adhesion molecule. J Cell Biol 114, 1059-1068 (1991).

177. Thoreson, M.A., Anastasiadis, P.Z., Daniel, J.M., Ireton, R.C., Wheelock, M.J., Johnson, K.R., Hummingbird, D.K. and Reynolds, A.B. Selective uncoupling of p120(ctn) from E-cadherin disrupts strong adhesion. J Cell Biol 148, 189-202 (2000).

178. Maddox, A.S. and Burridge, K. RhoA is required for cortical retraction and rigidity during mitotic cell rounding. J Cell Biol 160, 255-265 (2003).

179. Magie, C.R. and Martindale, M.Q. Cell-cell adhesion in the cnidaria: insights into the evolution of tissue morphogenesis. Biol Bull 214, 218-232 (2008).

180. Nimnual, A.S., Taylor, L.J. and Bar-Sagi, D. Redox-dependent downregulation of Rho by Rac. Nat Cell Biol 5, 236-241 (2003).

181. Bishop, A.L. and Hall, A. Rho GTPases and their effector proteins. Biochem $J$ 348 (Pt 2), 241-255 (2000).

182. Hakoshima, T., Shimizu, T. and Maesaki, R. Structural basis of the Rho GTPase signaling. J Biochem 134, 327-331 (2003).

183. Moon, S.Y. and Zheng, Y. Rho GTPase-activating proteins in cell regulation. Trends Cell Biol 13, 13-22 (2003).

184. Holinstat, M., Knezevic, N., Broman, M., Samarel, A.M., Malik, A.B. and Mehta, D. Suppression of RhoA activity by focal adhesion kinase-induced activation of p190RhoGAP: role in regulation of endothelial permeability. J Biol Chem 281, 2296-2305 (2006).

185. Bass, M.D., Morgan, M.R., Roach, K.A., Settleman, J., Goryachev, A.B. and Humphries, M.J. p190RhoGAP is the convergence point of adhesion signals from alpha 5 beta 1 integrin and syndecan-4. J Cell Biol 181, 1013-1026 (2008).

186. Bradley, W.D., Hernandez, S.E., Settleman, J. and Koleske, A.J. Integrin signaling through Arg activates p190RhoGAP by promoting its binding to p120RasGAP and recruitment to the membrane. Mol Biol Cell 17, 4827-4836 (2006). 
187. Grabocka, E. and Wedegaertner, P.B. Functional consequences of G alpha 13 mutations that disrupt interaction with p115RhoGEF. Oncogene 24, 2155-2165 (2005).

188. Chen, Z., Singer, W.D., Sternweis, P.C. and Sprang, S.R. Structure of the p115RhoGEF rgRGS domain-Galpha13/i1 chimera complex suggests convergent evolution of a GTPase activator. Nat Struct Mol Biol 12, 191-197 (2005).

189. Lang, P., Gesbert, F., Delespine-Carmagnat, M., Stancou, R., Pouchelet, M. and Bertoglio, J. Protein kinase A phosphorylation of RhoA mediates the morphological and functional effects of cyclic AMP in cytotoxic lymphocytes. EMBO J 15, 510-519 (1996).

190. Dong, J.M., Leung, T., Manser, E. and Lim, L. cAMP-induced morphological changes are counteracted by the activated RhoA small GTPase and the Rho kinase ROKalpha. J Biol Chem 273, 22554-22562 (1998).

191. Birukova, A.A., Zagranichnaya, T., Fu, P., Alekseeva, E., Chen, W., Jacobson, J.R. and Birukov, K.G. Prostaglandins PGE(2) and PGI(2) promote endothelial barrier enhancement via PKA- and Epac1/Rap1-dependent Rac activation. Exp Cell Res 313, 2504-2520 (2007).

192. O'Connor, K.L. and Mercurio, A.M. Protein kinase A regulates Rac and is required for the growth factor-stimulated migration of carcinoma cells. $J$ Biol Chem 276, 47895-47900 (2001).

193. Kawamura, S., Miyamoto, S. and Brown, J.H. Initiation and transduction of stretch-induced RhoA and Rac1 activation through caveolae: cytoskeletal regulation of ERK translocation. J Biol Chem 278, $31111-31117$ (2003).

194. Muranyi, A., Zhang, R., Liu, F., Hirano, K., Ito, M., Epstein, H.F. and Hartshorne, D.J. Myotonic dystrophy protein kinase phosphorylates the myosin phosphatase targeting subunit and inhibits myosin phosphatase activity. FEBS Lett 493, 80-84 (2001).

195. Kovacs, M., Toth, J., Hetenyi, C., Malnasi-Csizmadia, A. and Sellers, J.R. Mechanism of blebbistatin inhibition of myosin II. J Biol Chem 279, 35557-35563 (2004).

196. Schlegel, N. and Waschke, J. Impaired cAMP and Rac 1 signaling contribute to TNF-alpha-induced endothelial barrier breakdown in microvascular endothelium. Microcirculation 16, 521-533 (2009). 
197. Schlegel, N., Burger, S., Golenhofen, N., Walter, U., Drenckhahn, D. and Waschke, J. The role of VASP in regulation of cAMP- and Rac 1-mediated endothelial barrier stabilization. Am J Physiol Cell Physiol 294, C178-188 (2008).

198. Baumer, Y., Drenckhahn, D. and Waschke, J. cAMP induced Rac 1-mediated cytoskeletal reorganization in microvascular endothelium. Histochem Cell Biol 129, 765-778 (2008).

199. Qiao, J., Huang, F. and Lum, H. PKA inhibits RhoA activation: a protection mechanism against endothelial barrier dysfunction. Am J Physiol Lung Cell Mol Physiol 284, L972-980 (2003).

200. Birukova, A.A., Birukov, K.G., Adyshev, D., Usatyuk, P., Natarajan, V., Garcia, J.G. and Verin, A.D. Involvement of microtubules and Rho pathway in TGF-beta1-induced lung vascular barrier dysfunction. J Cell Physiol 204, 934-947 (2005).

201. Guo, F. and Zheng, Y. Rho family GTPases cooperate with p53 deletion to promote primary mouse embryonic fibroblast cell invasion. Oncogene 23, 5577-5585 (2004).

202. Garcia-Mata, R., Wennerberg, K., Arthur, W.T., Noren, N.K., Ellerbroek, S.M. and Burridge, K. Analysis of activated GAPs and GEFs in cell lysates. Methods Enzymol 406, 425-437 (2006).

203. Schmidt, A. and Hall, A. Guanine nucleotide exchange factors for Rho GTPases: turning on the switch. Genes Dev 16, 1587-1609 (2002).

204. Jones, C.A., Nishiya, N., London, N.R., Zhu, W., Sorensen, L.K., Chan, A.C., Lim, C.J., Chen, H., Zhang, Q., Schultz, P.G., Hayallah, A.M., Thomas, K.R., Famulok, M., Zhang, K., Ginsberg, M.H. and Li, D.Y. Slit2-Robo4 signalling promotes vascular stability by blocking Arf6 activity. Nat Cell Biol 11, 1325-1331 (2009).

205. Liu, D., Hou, J., Hu, X., Wang, X., Xiao, Y., Mou, Y. and De Leon, H. Neuronal chemorepellent Slit2 inhibits vascular smooth muscle cell migration by suppressing small GTPase Rac1 activation. Circ Res 98, 480-489 (2006).

206. Saharinen, P., Eklund, L., Miettinen, J., Wirkkala, R., Anisimov, A., Winderlich, M., Nottebaum, A., Vestweber, D., Deutsch, U., Koh, G.Y., Olsen, B.R. and Alitalo, K. Angiopoietins assemble distinct Tie 2 signalling complexes in endothelial cell-cell and cell-matrix contacts. Nat Cell Biol 10, 527-537 (2008). 
207. Fukuhara, S., Sako, K., Noda, K., Nagao, K., Miura, K. and Mochizuki, N. Tie2 is tied at the cell-cell contacts and to extracellular matrix by angiopoietin-1. Exp Mol Med 41, 133-139 (2009).

208. Sonnenberg, A., Calafat, J., Janssen, H., Daams, H., van der Raaij-Helmer, L.M., Falcioni, R., Kennel, S.J., Aplin, J.D., Baker, J., Loizidou, M. et al. Integrin alpha 6/beta 4 complex is located in hemidesmosomes, suggesting a major role in epidermal cell-basement membrane adhesion. J Cell Biol 113, 907-917 (1991).

209. Nejjari, M., Hafdi, Z., Dumortier, J., Bringuier, A.F., Feldmann, G. and Scoazec, J.Y. alpha6beta1 integrin expression in hepatocarcinoma cells: regulation and role in cell adhesion and migration. Int J Cancer 83, 518-525 (1999).

210. Delwel, G.O., de Melker, A.A., Hogervorst, F., Jaspars, L.H., Fles, D.L., Kuikman, I., Lindblom, A., Paulsson, M., Timpl, R. and Sonnenberg, A. Distinct and overlapping ligand specificities of the alpha $3 \mathrm{~A}$ beta 1 and alpha $6 \mathrm{~A}$ beta 1 integrins: recognition of laminin isoforms. Mol Biol Cell 5, 203-215 (1994).

211. Kim, Y., Kugler, M.C., Wei, Y., Kim, K.K., Li, X., Brumwell, A.N. and Chapman, H.A. Integrin alpha3beta1-dependent beta-catenin phosphorylation links epithelial Smad signaling to cell contacts. J Cell Biol 184, 309-322 (2009).

212. Wang, Z., Symons, J.M., Goldstein, S.L., McDonald, A., Miner, J.H. and Kreidberg, J.A. (Alpha)3(beta)1 integrin regulates epithelial cytoskeletal organization. J Cell Sci 112 (Pt 17), 2925-2935 (1999).

213. Hodivala-Dilke, K.M., DiPersio, C.M., Kreidberg, J.A. and Hynes, R.O. Novel roles for alpha3beta1 integrin as a regulator of cytoskeletal assembly and as a trans-dominant inhibitor of integrin receptor function in mouse keratinocytes. $J$ Cell Biol 142, 1357-1369 (1998).

214. Delaguillaumie, A., Lagaudriere-Gesbert, C., Popoff, M.R. and Conjeaud, H. Rho GTPases link cytoskeletal rearrangements and activation processes induced via the tetraspanin CD82 in T lymphocytes. J Cell Sci 115, 433-443 (2002).

215. Imhof, I., Gasper, W.J. and Derynck, R. Association of tetraspanin CD9 with transmembrane TGF \{alpha\} confers alterations in cell-surface presentation of TGF \{alpha\} and cytoskeletal organization. J Cell Sci 121, 2265-2274 (2008). 
216. Barreiro, O., Zamai, M., Yanez-Mo, M., Tejera, E., Lopez-Romero, P., Monk, P.N., Gratton, E., Caiolfa, V.R. and Sanchez-Madrid, F. Endothelial adhesion receptors are recruited to adherent leukocytes by inclusion in preformed tetraspanin nanoplatforms. J Cell Biol 183, 527-542 (2008).

217. Green, K.J. and Jones, J.C. Desmosomes and hemidesmosomes: structure and function of molecular components. Faseb J 10, 871-881 (1996).

218. Demali, K.A. Vinculin--a dynamic regulator of cell adhesion. Trends Biochem Sci 29, 565-567 (2004).

219. Linask, K.K., Manisastry, S. and Han, M. Cross talk between cell-cell and cell-matrix adhesion signaling pathways during heart organogenesis: implications for cardiac birth defects. Microsc Microanal 11, 200-208 (2005).

220. Crampton, S.P., Wu, B., Park, E.J., Kim, J.H., Solomon, C., Waterman, M.L. and Hughes, C.C. Integration of the beta-catenin-dependent Wnt pathway with integrin signaling through the adaptor molecule Grb2. PLoS One 4, e7841 (2009).

221. Brose, K., Bland, K.S., Wang, K.H., Arnott, D., Henzel, W., Goodman, C.S., Tessier-Lavigne, M. and Kidd, T. Slit proteins bind Robo receptors and have an evolutionarily conserved role in repulsive axon guidance. Cell 96, 795-806 (1999).

222. Tseng, R.C., Lee, S.H., Hsu, H.S., Chen, B.H., Tsai, W.C., Tzao, C. and Wang, Y.C. SLIT2 attenuation during lung cancer progression deregulates beta-catenin and E-cadherin and associates with poor prognosis. Cancer Res 70, 543-551 (2010). 


\section{VITA}

Feng Zhang was born in Qufu City, Shandong Province at P.R.China in 1979. In September 1997, he attended Yantai University for undergraduate studies, majoring in Biochemistry. After achieving the degree of Bachelor of Science in July 2001, he joined Nanjing University in September 2001 for master graduate studies majoring in Biochemistry and Molecular Biology, and obtained the degree of Master of Science in July 2004. In August 2005, he entered the Integrated Program in Biomedical Sciences at the University of Tennessee Health Science Center for Ph.D. studies. In July 2006, he joined Dr. Xin A. Zhang's laboratory in Department of Medicine and conducted research in the functions of CD151 in vascular stability. In 2009, he received a predoctoral fellowship from American Heart Association. He received his Doctor of Philosophy degree from the University of Tennessee in December of 2010. 